\title{
The EFSUMB Guidelines and Recommendations for the Clinical Practice of Contrast-Enhanced Ultrasound (CEUS) in Non-Hepatic Applications: Update 2017 (Long Version) \\ Die EFSUMB-Leitlinien und Empfehlungen für den klinischen Einsatz des kontrastverstärkten Ultraschalls (CEUS) bei nicht-hepatischen Anwendungen: Update 2017 (Langversion)
}

Authors

Paul S. Sidhu ${ }^{1}$, Vito Cantisani ${ }^{2}$, Christoph F. Dietrich ${ }^{3}$, Odd Helge Gilja ${ }^{4}$, Adrian Saftoiu' ${ }^{5}$, Eva Bartels ${ }^{6}$, Michele Bertolotto ${ }^{7}$, Fabrizio Calliada ${ }^{8}$, Dirk-André Clevert ${ }^{9}$, David Cosgrove ${ }^{10}$, Annamaria Deganello ${ }^{1}$, Mirko D’Onofrio ${ }^{11}$,

Francesco Maria Drudi ${ }^{12}$, Simon Freeman ${ }^{13}$, Christopher Harvey ${ }^{14}$, Christian Jenssen ${ }^{15}$, Ernst-Michael Jung ${ }^{16}$, Andrea Sabine Klauser ${ }^{17}$, Nathalie Lassau ${ }^{18}$, Maria Franca Meloni ${ }^{19}$, Edward Leen ${ }^{20}$, Carlos Nicolau ${ }^{21}$, Christian Nolsoe ${ }^{22}$, Fabio Piscaglia' ${ }^{23}$, Francesco Prada ${ }^{24}$, Helmut Prosch ${ }^{25}$, Maija Radzina ${ }^{26}$, Luca Savelli27, Hans-Peter Weskott ${ }^{28}$, Hessel Wijkstra ${ }^{29}$

Affiliations

1 Department of Radiology, King's College London, King's College Hospital, London, United Kingdom of Great Britain and Northern Ireland

2 Department of Radiology, Policlinico Umberto I, Univ. Sapienza of Rome, Italy

3 Med. Klinik 2, Caritas-Krankenhaus, Bad Mergentheim, Germany and Department of Ultrasound, The First Affiliated Hospital Zhengzhou University, China

4 National Centre for Ultrasound in Gastroenterology, Haukeland University Hospital, Bergen, and Department of Clinical Medicine, University of Bergen, Norway

5 Research Center of Gastroenterology and Hepatology, University of Medicine and Pharmacy of Craiova, Romania

6 Center for Neurological Vascular Diagnostics, München, Germany

7 Department of Radiology, University of Trieste, Italy

8 Department of Radiology, University of Pavia, Policlinico San Matteo, Pavia, Italy

9 Interdisciplinary Ultrasound-Center, Department of Radiology, University of Munich - Grosshadern Campus, Munich, Germany

10 Clinical Sciences, Imperial College, London, United Kingdom of Great Britain and Northern Ireland

11 Department of Radiology, GB Rossi University Hospital, University of Verona, Verona, Italy

12 Department of Radiology, University La Sapienza, Italy

13 Department of Imaging, Derriford Hospital, Plymouth, United Kingdom of Great Britain and Northern Ireland

14 Department of Imaging, Imperial College Health Trust, London, United Kingdom of Great Britain and Northern Ireland

15 Department of Internal Medicine, Krankenhaus Märkisch Oderland Strausberg/Wriezen, Strausberg, Germany

16 Radiologie, Universitätsklinikum Regensburg, Germany

17 Universitaetsklinik fuer Radiodiagnostik, Medizinische Universitaet Innsbruck, Austria
18 Gustave Roussy Cancer Campus. Imaging Department and IR4M. UMR8081. Université Paris-Sud, Université ParisSaclay, Paris, France

19 Casa Di Cura Igea, Department of Interventional Ultrasound, Milan, Italy

20 Imaging Department, Imperial College London, United Kingdom of Great Britain and Northern Ireland

21 Radiology Department, Hospital Clinic, Barcelona, Spain

22 Ultrasound Section, Division of Surgery, Department of Gastroenterology, Herlev Hospital. Copenhagen Academy for Medical Education and Simulation (CAMES), University of Copenhagen, Denmark

23 Department of Medical and Surgical Sciences, Division of Internal Medicine, Bologna, Italy

24 Department of Neurosurgery, Fondazione IRCCS Istituto Neurologico C. Besta, Milan, Italy and Department of Neurological Surgery, University of Virginia Health Science Center, Charlottesville, VA, USA, Milan, Italy

25 Abteilung für Allgemeine Radiologie und Kinderradiologie, Medizinische Universität Wien, Austria

26 Paula Stradina Clinical University Hospital, Diagnostic Radiology Institute, Riga Stradins University, Radiology Research Laboratory, Riga, Latvia

27 Gynecology and Early Pregnancy Ultrasound Unit, Department of Obstetrics and Gynecology, University of Bologna, Italy

28 Ultrasound, Krankenhaus Siloah, Hannover, Germany 29 Urology, AMC University Hospital, Amsterdam and Signal Processing Systems, Eindhoven University of Technology, The Netherlands

Key words vascular, urinary tract, neurology, musculoskeletal system, head/neck 
received 03.07.2017

accepted 05.12.2017

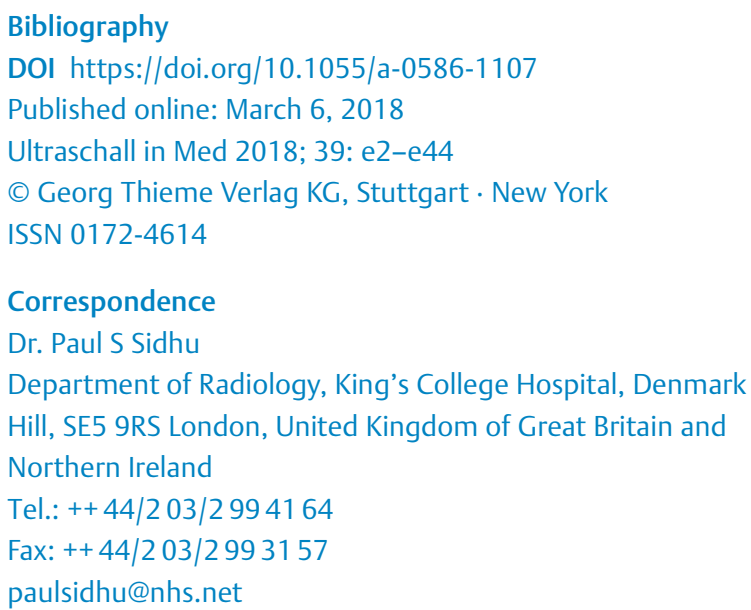

\section{ABSTRACT}

The updated version of the EFSUMB guidelines on the application of non-hepatic contrast-enhanced ultrasound (CEUS) deals with the use of microbubble ultrasound contrast outside the liver in the many established and emerging applications.

\section{ZUSAMMENFASSUNG}

Die aktualisierte Version der EFSUMB-Leitlinien für die Anwendung von nicht-hepatischem kontrastverstärktem Ultraschall (CEUS) befasst sich mit der Verwendung von Mikrobläschen Ultraschall-Kontrastmitteln außerhalb der Leber in zahlreichen etablierten und neu entstehenden Einsatzbereichen.

\section{Introduction and general considerations}

Previous contrast-enhanced ultrasound (CEUS) documents from the European Federation of Societies for Ultrasound in Medicine and Biology (EFSUMB) encompassing hepatic [1 - 3] and nonhepatic applications [4] have been published with a statement on CEUS use in pediatric applications [5]. The present document reflects the current applications in non-hepatic CEUS and updates the previous EFSUMB guidelines published in 2012 [4]. The EFSUMB guidelines on CEUS are intended to inform clinical practice rather than to report on research projects. Thus, they are a digest of current findings formulated by a group of experts and are primarily based on surveys of the published peer-reviewed literature (so that abstracts and conference proceedings are excluded). Levels of evidence (LoE) and grade of recommendation (GoR) are formulated and presented to the reader to enable comprehensive understanding of the current clinical status of each CEUS application and based on the criteria used as in previous EFSUMB guidelines; levels of evidence and grades of recommendations are assigned according to the Oxford Centre for Evidence-based Medicine criteria (http://www.cebm.net/oxford-centre-evidencebased-medicine-levels-evidence-march-2009/). A consensus opinion was established by vote as follows: strong consensus (>95\%), broad consensus (75-95\%), with approval, disapproval or abstaining from each participant. It is important to consider that nearly all applications contained in the current guidelines are "off-label" and are likely to remain so for some time. This does not present an impediment to the use of ultrasound contrast agents (UCAs) when applied outside licensing, a topic detailed in an accompanying article to previous guidelines [6]. Indeed the EFSUMB guidelines provide the evidence to incorporate UCAs into clinical practice despite being "off-label", influencing regulatory authorities to sanction use as recently demonstrated by the Food and Drug Administration of the United States of America approval of UCAs in pediatric practice [7-9].
In general, CEUS is most useful where an abnormality can be displayed on B-mode ultrasound (US), and the better the quality of the B-mode imaging, the better the quality of the CEUS images. Importantly, CEUS is always used as an extension of conventional US (B-mode and color Doppler). Contrast studies should always be interpreted in the context of the overall clinical picture, other imaging and laboratory tests.

Overall, UCAs are mainly used as vascular agents following intravenous injection and they highlight the macro- and microvascular systems. However, they can also be instilled into body cavities, both normal and pathological. Instillation into the urinary bladder for vesicoureteral reflux is a classic example. Other examples include instillation into drainage catheters to define their position, the extent of the cavity and its continuity. Intradermal injection is used as a form of lymphangiography, with the UCA being spontaneously taken up into the lymphatics as an extension of their normal particle trapping activity. It is used to highlight sentinel lymph nodes, chiefly in breast cancer.

\section{Investigator training}

One of the central strategies of EFSUMB is to ensure high-quality US education and sustain excellent professional standards in CEUS training and practice. Previously, EFSUMB defined three levels of training requirements in a minimal training standards document [10], with specific reference to CEUS in Appendix 14 [11]. EFSUMB recommends that CEUS should be performed by operators that have achieved competence Level 1 , as it has been recognized that the diagnostic performance of CEUS is dependent on the observer's level of experience [12]. Accordingly, appropriate training and education is strongly advised for every investigator who performs CEUS examinations [13]. Furthermore, investigators should ensure that their US scanning machine is optimized for CEUS acquisition and the post-processing of data. The operator must gain sufficient knowledge of indications and contraindica- 
tions of CEUS and training in ultrasound contrast agent administration and perform CEUS within the medico-legal framework of each individual country.

\section{RECOMMENDATION 1}

The operator must gain sufficient knowledge and training in CEUS, ultrasound contrast agent administration and contraindications, and perform the examination within the medicolegal framework of each individual country (LoE 5, GoR C). Strong consensus (20/0/0, $100 \%)$

\section{Terminology}

\section{Equipment}

Ultrasound equipment based on contrast-specific ultrasound modes is needed for CEUS examinations, based on the separation between non-linear response induced by microbubble UCA oscillations and linear US signal reflected by tissues [4]. In order to decrease the non-linear harmonic US signals generated by the tissues themselves, a low acoustic pressure is generally used, based on a low mechanical index (MI). Generally, a low MI examination is typically considered below 0.3 in order to minimize microbubble disruption, but also reduce tissue harmonics and artifacts. Nevertheless, most of the US systems are able to perform CEUS examinations with lower values of the MI, even 0.08 or 0.05 , and $\mathrm{MI}$ values vary with the different US manufacturers.

\section{Terminology}

Ultrasound contrast agents are used for enhancement of the US signal from flowing blood as they are limited to the blood vessels (blood pool UCA) [3, 4]. They were initially developed to enhance the Doppler US signals, based on higher MI techniques as opposed to the currently widely applied low MI specific modes. During high MI Doppler modes, injection of a UCA as a bolus produces "blooming", due to flash or movement artifacts, which are not visible using specific harmonic imaging modes. The CEUS acronym has been introduced by EFSUMB and is generally accepted as the official term describing contrast enhanced ultrasonography techniques [3, 4]. Low MI techniques are preferred to the high MI techniques based on Doppler or power Doppler modes [14-16]. Most of the ultrasound systems have a dual split-screen display setting, with the low MI CEUS image shown alongside a conventional B-mode image. In the CEUS window, only a few signals from intensely reflective structures (e. g. calcifications or interfaces that produce large differences in acoustic impedance) should be seen, dependent on the settings of the MI and gain. Modes with a single screen display can also be used, where the CEUS image is displayed as a color overlay on the conventional B-mode image.

Each examined lesion should be described in terms of enhancement, taking into account the temporal behavior, degree of enhancement as compared with the surrounding tissues (non- enhanced, hypo-enhanced, iso-enhanced or hyper-enhanced), as well as the contrast distribution (homogeneity or heterogeneity). Two phases are described for most organs that have a single arterial blood supply (except the liver and lungs) [3, 4]:

a) the arterial phase starts from around $10-20$ seconds until around $35-40$ seconds after contrast injection, showing a progressive degree of enhancement;

b) the venous phase starts from around 30 to 45 seconds after contrast injection, showing a plateau and then a progressive decrease.

\section{Safety}

UCAs are administered safely in various applications with minimal risk to patients $[4,17-20]$. They are not excreted through the kidneys, and can be safely administered to patients with renal insufficiency with no risk of contrast-related nephropathy or nephrogenic systemic fibrosis. There is no need for blood tests prior to UCA injection, and there is no evidence of any effect on thyroid function, as UCAs do not contain iodine. UCAs have a very low rate of anaphylactoid reactions (1:7000 patients, $0.014 \%)[17,21,22],[20]$ significantly lower than the rate with iodinated state-of-the-art CT agents (35-95:100 000 patients, $0.035-0.095 \%$ ) [23], comparable to the rate of severe anaphylactoid reactions associated with gadolinium-based contrast agents at $0.001-0.01 \%$ [24]. Serious anaphylactoid reactions to UCAs are observed in approximately 1:10 000 exposures [4, 20].

Data from 75 completed studies (pooled data from 6307 patients) in North America, Europe, and Asia showed that the most frequent adverse events were headache $(2.1 \%)$, nausea $(0.9 \%)$, chest pain $(0.8 \%)$ and chest discomfort $(0.5 \%)$. All other adverse events occurred at a frequency of $<0.5 \%$. Most adverse events were mild and resolved spontaneously within a short time without sequelae. In most cases allergy-like events and hypotension occurred within a few minutes following the injection of the UCA. The overall reported rate of fatalities attributed to one UCA, SonoVue ${ }^{\mathrm{TM}}$ (Bracco, Milan), is low (14/2447 083 exposed patients; $0.0006 \%$ ) and compares favorably with the risk for fatal events reported for iodinated contrast agents (approximately $0.001 \%$ ). In all reported fatalities after use of a UCA, in both cardiac and noncardiac cases, an underlying patient medical circumstance played a major role in the fatal outcome [25]. The intravesical administration of UCAs has been evaluated in a total of 7082 children described in 15 studies and in a European survey of 4131 children with $0.8 \%$ reported adverse events, mostly related to bladder catheterization [26].

Contrast-enhanced ultrasound is also used off-label in the pediatric population [5], and in renal assessment [27, 28], and in numerous other documented areas [4]. The Food and Drug Administration (FDA) in the United States of America (USA) recently approved the use of Lumason ${ }^{\mathrm{TM}}$ (marketed as SonoVue ${ }^{\mathrm{TM}}$ Bracco, Milan, outside the USA) for pediatric liver imaging [7, 8], which is an important development in pediatric imaging. A significant reduction of ionizing radiation exposure can be achieved in many areas by using CEUS in pediatric patients [5, 29, 30]. 


\section{RECOMMENDATION 2}

Intravenous CEUS use is safe and effective in both adult and pediatric populations (LoE 2a, GoR B). Strong consensus $(20 / 0 / 0,100 \%)$

\section{RECOMMENDATION 3}

Intracavitary use of ultrasound contrast agents is safe (LoE $1 \mathrm{~b}$, GoR B). Strong consensus (20/0/0, 100\%)

\section{Genitourinary}

\section{Bladder}

\section{Background}

Noninvasive diagnostic imaging may play a role in urinary bladder tumors, but cannot replace cystoscopy and pathologic staging. The depth of wall invasion, the histological grade and the extension outside the bladder are main factors determining prognosis and therapeutic approach.

\section{Study procedure}

Optimal bladder filling (approximately $2 / 3$ of the total bladder volume) is critical [31]. Insufficient filling prevents lesion detection, while excessive distension results in bladder wall thinning and reduced conspicuity of the wall layer, making it difficult to differentiate a superficial from an infiltrating lesion [32]. The layers of the bladder wall can be differentiated after UCA administration; the mucosa, and particularly the submucosal layer, exhibit early and intense enhancement that persists for $1-2$ minutes [31], whereas the muscular layer has lesser and delayed enhancement.

\section{Image interpretation}

\section{Characterization of mural lesions}

CEUS improves the differential diagnosis of intraluminal lesions, allowing the detection of tumors, which are vascularized and enhance [33, 34], in contrast to non-enhancing hematomas [34]. In 35 patients with cystoscopy and biopsy as the reference standard, CEUS correctly assessed tumor presence or absence in $88 \%$ of cases [35].

\section{Bladder tumor staging}

CEUS is superior to conventional B-mode US for identifying infiltration of the muscle layer [31], but magnetic resonance (MR) and computed tomography (CT) imaging are essential for the local staging of bladder tumors. The ability to predict tumor grading based on the pattern of CEUS enhancement remains under evaluation $[32,36]$.

\section{Limitations}

In patients with anatomical circumstances leading to poor urinary bladder visualization, CEUS cannot always provide the desired information. Similar to MR and CT imaging of bladder tumor detection, an important limitation of CEUS is the difficulty in identifying both small $(<1 \mathrm{~cm})$ lesions and large flat, plaque-like tumors. Tumor position can affect the quality of CEUS depiction and the accuracy of staging. Tumors in the anterior portion of the bladder dome are sometimes difficult to visualize. Columnar hypertrophy of the bladder wall and prostatic hypertrophy can hide or mimic urothelial polypoid projections [31]. Benign tumors and focal cystitis are other uncommon conditions that present with focal bladder wall enhancement and can mimic a malignant lesion. CEUS is unable to provide a panoramic bladder view, as in the case of CT and MR imaging.

\section{RECOMMENDATION 4}

The most useful application of CEUS is the differential diagnosis of bladder cancer from hematoma in patients with hematuria when the diagnosis is equivocal on conventional B-mode and Doppler US (LoE 2b, GoR C). Strong consensus (20/0/0, $100 \%$ )

\section{Kidney}

\section{Background}

Ultrasound is the preferred imaging modality in patients with known or suspected renal disease for assessing renal size, detecting focal lesions and obstruction of the collecting system and for identifying vascular disorders but it cannot definitively distinguish between benign and malignant lesions. Doppler US helps to characterize renal blood flow, with limitations of attenuation, low sensitivity for very slow blood flow, and angle dependency.

\section{Study procedure}

The kidneys enhance rapidly and intensely after UCA administration, with potential to assess both the macro- and the microvasculature, the former immediately after UCA arrival. The arterial pedicle and main branches enhance first, followed rapidly by the segmental, interlobar, arcuate and interlobular arteries and then complete cortical enhancement. Medullary enhancement follows, with the outer medulla enhancing first, followed by gradual fill-in of the pyramids [37]. As UCAs are not excreted by the kidneys, there is no UCA in the renal collecting system. With CEUS only two enhancement phases occur: a cortical phase, $15-30 \mathrm{~s}$ after UCA administration with cortical enhancement seen, and a parenchymal phase, where both cortex enhancement and medulla enhancement occur $25 s-4$ mins after UCA administration. There is normally excellent depiction of renal perfusion throughout the kidney, superior to Doppler US. Contrast enhancement is reported to be less intense and fades earlier in patients with chronic renal disease [38, 39]. 


\section{Renal Ischemia}

Excellent diagnostic performance of CEUS in the detection of renal parenchymal ischemia, similar to that of CT imaging and superior to color Doppler US, has been reported. Infarcts appear as wedgeshaped non-enhancing areas within an otherwise enhanced kidney [40]. The excellent spatial resolution of CEUS allows clear differentiation between renal infarction and cortical necrosis, which appears as non-enhancing cortical areas with preserved hilar vascularity [37, 40, 41]. Differentiation between hypoperfused and non-perfused areas is clear following UCA administration; only infarcted areas completely lack contrast enhancement.

\section{Renal Focal Lesions}

\section{Differential diagnosis between solid renal masses and pseudotumors}

CEUS is used to differentiate between renal tumors and mimicking anatomical variations not characterized with B-mode and conventional Doppler US. Pseudotumors have the same enhancing characteristics as the surrounding parenchyma in all phases $[37,42]$, while the enhancement in renal tumors in the majority of cases differs from the surrounding parenchyma, with a difference in the degree or distribution of enhancement in at least one vascular phase. Renal tumors, however, do not show specific perfusion patterns. Virtually iso-enhancing tumors in all vascular phases are encountered in up to $5 \%$ of solid renal lesions. A normal perfusion pattern on CEUS is a major criterion for the differential diagnosis between an iso-enhancing renal lesion and a pseudotumor. A pseudotumor demonstrates the vascular architecture of normal renal parenchyma, displayed during the early arterial phase, with branching from the hilum to the periphery without disruption of vessels or aberrant vessels.

\section{Characterization of complex cystic renal masses}

CEUS is appropriate in the Bosniak classification of renal cysts and is suggested to be superior to $\mathrm{CT}$ imaging for detecting additional septa, thickening of the wall or septa, and solid components [28, $43-46]$. CEUS allows the characterization of renal cystic lesions as benign or malignant with at least the same accuracy as CT imaging, but CT remains the reference method for staging patients with malignant cystic lesions. CEUS is well suited for the followup of non-surgical complex cystic lesions and has potential to replace $C T$. The absence of ionizing radiation is advantageous. The presence of lesion calcification hampers CEUS evaluation of complex cysts masses [28, $43-46]$.

\section{Characterization of indeterminate renal masses}

In clinical practice, most abdominal CT imaging studies are not performed with a specific renal protocol to characterize renal lesions, frequently indeterminate renal lesions are identified. Follow-up US assessment should be comprehensive, including CEUS, to obviate an unnecessary correctly protocoled repeat CT study. Bmode US can determine the presence of a simple benign cyst. CEUS is more sensitive than CT for detecting blood flow in hypovascularized lesions and can be used to distinguish between com- plex cysts and solid lesions, particularly those which remain unresolved after CT imaging, B-mode and color Doppler US [28, 47].

\section{Renal infections}

The diagnosis of acute uncomplicated pyelonephritis is based on clinical examination and laboratory findings. Conventional B-mode US is used to exclude urinary obstruction and renal calculi. Additional investigations should be considered if the patient remains febrile following 72 hours of treatment. In these patients, with complicated pyelonephritis, CEUS is effective in identifying inflammatory involvement, characterized by round or wedgeshaped hypovascular parenchymal areas, most conspicuous during the parenchymal late phase. An abscess is manifested as a non-enhancing area, with or without rim or septal enhancement, solitary or within areas of pyelonephritis. CEUS can be used to monitor the resolution of abscesses, which can be prolonged, even with clinical improvement [48].

\section{Evaluation of solid renal lesions}

A number of studies have attempted to evaluate the differentiation of renal tumors, particularly angiomyolipoma and renal cell carcinoma, by means of different features of time-intensity curves after UCA administration. The majority of angiomyolipomas are reliably differentiated with $C T$ or MR imaging and although results are promising with CEUS, overlap with both qualitative and quantitative analyses with different tumors is evident. In expert hands, CEUS may help identify renal vein invasion by cancer, as the arterial vascularization of the thrombus may differentiate bland thrombus (non-enhancing) from tumor invasion (enhancing thrombus) [49].

\section{RECOMMENDATION 5}

CEUS can be used to diagnose ischemic renal disorders, such as infarction (LoE 1b, GoR A). Strong consensus (20/0/0, $100 \%)$

\section{RECOMMENDATION 6}

CEUS can differentiate between renal tumors and anatomical variants mimicking a renal tumor ("pseudotumors") when conventional US is equivocal (LoE 1b, GoR A). Strong Consensus $(19 / 0 / 1,100 \%)$

\section{RECOMMENDATION 7}

CEUS can be used to characterize complex cysts according to the Bosniak criteria (LoE 1b, GoR A). Broad Consensus (15/2/3, $88 \%$ ) 


\section{RECOMMENDATION 8}

CEUS can be used to characterize indeterminate renal lesions (LoE 1b, GoR A). Strong Consensus (19/0/1, 100\%)

\section{RECOMMENDATION 9}

CEUS can be used for the identification of renal abscesses in complicated acute pyelonephritis (LoE 1b, GoR A). Strong consensus $(20 / 0 / 0,100 \%)$

\section{RECOMMENDATION 10}

CEUS can be used for the follow-up of non-surgical renal lesions (LoE 4, GoR C). Strong consensus (20/0/0, $100 \%$ )

\section{Vesicoureteral Reflux (VUR)}

\section{Background}

Conventional voiding cystourethrography remains the gold standard for the detection of VUR, notwithstanding ionizing radiation concerns, despite contrast-enhanced voiding urosonography (ceVUS) being the superior option. Many early comparative studies between ceVUS and cystourethrography were obtained with Levovist ${ }^{\mathrm{TM}}$ (Schering AG, Berlin), now no longer available UCA. SonoVue ${ }^{\mathrm{TM}}$ (Bracco SpA, Milan), recently licensed for this purpose, performs comparatively well, and has a favorable safety profile in children [50] with high diagnostic performance for the detection of reflux and for assessment of the urethra [51 - 59].

\section{Study procedure}

The basic steps of ceVUS are [50]:

a) B-mode US evaluation of the kidneys and bladder

b) Intravesical administration of UCA diluted in normal sterile saline

c) Repeated imaging of the bladder and kidneys with CEUS during and after bladder filling and while voiding

d) During voiding urethrosonography (transpubic and/or transperineal) may be added [60].

UCA can be administered via a transurethral bladder catheter or via suprapubic puncture $\left(0.1-0.5 \mathrm{~mL}\right.$ SonoVue ${ }^{\mathrm{TM}}$ in $500 \mathrm{~mL} 0.9 \%$ saline), by slow instillation during CEUS monitoring, until adequate enhancement of the bladder content is achieved; dose adjustment with excessive shadowing or insufficient signal. A full bladder is necessary for suprapubic puncture.

\section{Diagnosis of vesicoureteral reflux}

Reflux is diagnosed when the UCA appears in one or both ureters and/or the pelvicalyceal system. Vesicoureteral reflux is graded $\mathrm{I}-\mathrm{V}$ depending on severity, analogous to the international reflux grading system of voiding cystourethrography [61]. US imaging is continued during and after voiding with the child supine, prone, sitting, or standing, always imaging the kidneys and bladder alternately as the position allows [51].

Contrast-enhanced voiding urosonography has a higher rate of vesicoureteral reflux detection compared to voiding cystourethrography, as ceVUS is more sensitive to the detection of small amounts of refluxed UCA [56, 57, 62]. Moreover, ceVUS imaging is continuous, while fluoroscopy is intermittent with cystourethrography, allowing better detection of intermittent reflux on CEUS. Notably, reflux episodes missed on voiding cystourethrography but detected with ceVUS tend to be higher grade, and of greater clinical concern $[56,57,62]$. The ability to detect clinically important reflux and the lack of ionizing radiation support the use of ceVUS for initial diagnostic and follow-up evaluation of VUR in boys and girls, as well as screening of high-risk patients. A limitation of ceVUS is the inability to image the entire urinary tract simultaneously. Furthermore, ceVUS is not recommended as the primary imaging modality for reflux, if the bladder or one of the kidneys is not depicted on US, for specific urethral and/or bladder functional and anatomical evaluation and when imaging is required for detailed anatomical assessment, e.g. in the evaluation of recto-urethral fistulas in neonates with anorectal malformation [52]. The urethra may also be evaluated effectively both in girls and in boys. Although evidence is limited, the technique is promising [55, 60, 62-64].

Contrast-enhanced voiding urosonography has been used for vesicoureteral reflux in renal transplant recipients with recurrent urinary tract infections [65-67], both in adults and children. In 23 adult renal transplant recipients, ceVUS was compared with radionuclide cystography [65], in 37 adult patients ceVUS was compared with conventional voiding cystourethrography [66] and in $27 \mathrm{pa}-$ tients ( 8 children or adolescents, 19 adults) cycling ceVUS (i.e., obtained by filling the bladder and having the patient void around the urinary bladder catheter two times) was compared with ceVUS in the first cycle [67]. Results indicated that ceVUS was highly effective in detecting vesicoureteral reflux in adult renal transplant recipients. Compared to techniques involving exposure to ionizing radiation, the sensitivity and specificity ranged between $75 \%$ $93 \%$ and $71 \%-95 \%$, respectively [66]. Compared with the first cycle, cyclic ceVUS did not improve detection sensitivity for vesicoureteral reflux, but revealed higher grades of reflux [67].

\section{RECOMMENDATION 11}

Contrast-enhanced voiding urosonography should be the initial examination for suspected vesicoureteral reflux in girls (LoE 1a, GoR A) and in boys (LoE 2b, GoR B). Strong Consensus $(19 / 0 / 1,100 \%)$

\section{RECOMMENDATION 12}

Contrast-enhanced voiding urosonography should be used in the follow-up of vesicoureteral reflux in girls and boys after conservative or surgical treatment. (LoE 1a, GoR A). Strong consensus $(20 / 0 / 0,100 \%)$ 


\section{RECOMMENDATION 13}

Contrast-enhanced voiding urosonography should be used to screen high-risk patients for reflux (e. g., siblings, transplanted kidney) (LoE 1a, GoR A). Strong Consensus (19/0/1, $100 \%)$

\section{Scrotum}

\section{Background}

Despite US being the imaging modality of choice for examination of the scrotum, findings may be equivocal and misinterpretation can result in an unnecessary orchiectomy. A challenge is the unequivocal differentiation between hypovascular and avascular lesions, presuming that an avascular lesion implies benign disease, which may be impossible on color Doppler US. CEUS provides a practical solution by increasing the confidence of the interpretation of lesion vascularity and of scrotal and cord vessels, allowing for appropriate clinical management.

\section{Study procedure}

A B-mode and color Doppler US examination of the lesion with linear high-frequency transducers should be performed to relate to the subsequent CEUS findings. A higher UCA concentration is required to examine the scrotal contents; typically $4.8 \mathrm{~mL}$ of SonoVue ${ }^{\mathrm{TM}}$ (Bracco SpA, Milan) [68]. The arterial phase in CEUS is the most important aspect of the examination. The testis and epididymis enhance rapidly but the arrival time varies between individuals. The arteries enhance first, followed within seconds by complete parenchymal enhancement. The scrotal wall tends to enhance to a lesser degree than the contents. There is no accumulation of UCA in the parenchyma of the testis and the enhancement declines over a variable period of time such that there is minimal residual enhancement by three minutes.

\section{Patterns of disease}

\section{Torsion of the spermatic cord}

The sensitivity of color Doppler US with current equipment for the identification and diagnosis of spermatic cord testicular torsion is adequate, even in the small volume testes of children [69]. In a small series of men with spermatic cord torsion, CEUS confirmed the absence of vascularization, but failed to add any clinically significant information to unenhanced color Doppler US [70]. There is no data to recommend the use of CEUS in spermatic cord torsion, although the absence of global vascularity can be clearly depicted [71].

\section{Segmental Infarction}

The appearance of acute segmental testicular infarction on conventional B-mode and color Doppler US is variable [72, 73]. Often the benign nature of the lesion is established by its wedge shape with markedly diminished or absent color Doppler flow [72]. The main concern is the differentiation of a segmental infarction with a rounded configuration from a poorly vascularized tumor [74]. CEUS improves the characterization of segmental infarction by demonstrating one or more ischemic parenchymal lobules separated by normal testicular vessels $[75,76]$. Subacute segmental infarction characteristically exhibits a perilesional rim of enhancement, which diminishes over time and is eventually lost with changes in lesion shape and shrinkage $[75,77]$.

\section{Trauma}

Conventional B-mode and color Doppler assessment of the testis in trauma is well established but underestimates the extent of injury [78]. Besides integrity or interruption of the tunica albuginea, the most important information for the surgeon is the extent of viable testicular tissue, an evaluation which is often difficult with conventional Doppler US because the injured testis is often hypovascular even in viable regions, as a consequence of testicular edema compromising vascular flow. CEUS allows delineation between the non-enhancing devascularized tissue and the enhancing viable parenchyma, enabling organ-sparing treatment. Moreover, CEUS offers a clear delineation of fracture lines and intratesticular hematomas [79-82].

\section{Inflammation}

Epididymo-orchitis is a clinical diagnosis and is usually easily confirmed on color Doppler US. Abscess formation is relatively common in cases of severe epididymo-orchitis, whereas venous infarction is exceedingly rare, thought to be a consequence of local swelling occluding the venous drainage of portions of the testis or of the entire testis $[71,76]$. CEUS may be used in selected cases of severe epididymo-orchitis. It allows unequivocal assessment of the presence or absence of vascular supply within focal testicular lesions. However, since both infarction and intratesticular abscess lack internal vessels, absolute differentiation remains difficult. CEUS may be able to determine the development of an abscess at an earlier stage, or the complete extent of a large abscess, and allow for prompt treatment $[70,71,76,83]$.

\section{Tumors and complex cysts}

The current understanding is that testicular tumors with a diameter of less than $1.5 \mathrm{~cm}$ may not show flow on color Doppler US and thus may be misinterpreted as a benign lesion, the purported hallmark of malignancy being an increase in vascularity [84]. Simple testicular cysts are usually benign, but any wall irregularity or echogenic debris may be suggestive of a (rare) cystic testicular tumor $[85,86]$. CEUS is able to confirm the absence of vascularity in benign complex cysts and epidermoid cysts $[87,88]$. It is thought that virtually all testicular tumors display vascularization on CEUS, with the exception of any cystic component and regions of necrosis. Very rare exceptions may be represented by extensively necrotic lesions, and by the so-called "burned out" testicular tumor [89-91].

\section{Evaluation of solid testicular lesions}

Several investigators have discussed the possibility of differentiating testicular tumors with CEUS, particularly between a malignant seminoma and a benign Leydig cell tumor. Using time-intensity curves, evaluating the wash-in and washout curves may help dis- 
tinguish malignant from benign tumors, with a prolonged washout observed in Leydig cell tumors [90], and reported rapid wash-in and raised enhancement for Leydig cell tumors in comparison seminoma $[92,93]$. Although these results are promising, both qualitative and quantitative CEUS analyses overlap between different histological types. Quantification of CEUS of testicular tumors remains a research tool. There is limited use of CEUS in intrascrotal extratesticular focal lesions, with no evidence regarding the usefulness for the differentiation of solid lesions [83].

\section{Spontaneous intratesticular hematoma}

Testicular hematoma can rarely present with acute scrotal pain in a patient with no history of trauma. US demonstrates an intratesticular mass suggesting malignancy, but the lack of enhancement is a good marker for the absence of vascularity and for a benign lesion, leading to a presumptive diagnosis and conservative management $[82,94,95]$.

\section{RECOMMENDATION 14}

CEUS can distinguish vascularized from non-vascularized focal testicular lesions, helping to exclude malignancy (LoE 1a, GoR A). Strong consensus (20/0/0, $100 \%)$

\section{RECOMMENDATION 15}

Testicular CEUS can discriminate non-viable regions in testicular trauma (LoE 2b, GoR B). Strong consensus (20/0/0, $100 \%$ )

\section{RECOMMENDATION 16}

CEUS can identify segmental infarction (LoE 2b, GoR B). Strong consensus $(20 / 0 / 0,100 \%)$

\section{RECOMMENDATION 17}

CEUS can identify abscess formation and infarction in severe epididymo-orchitis (LoE 2b, GoR B). Strong Consensus (18/0/ $2,100 \%)$

\section{Prostate Cancer}

\section{Background}

Conventional B-mode and Doppler transrectal US imaging have a limited role in the detection of prostate cancer because of poor sensitivity and specificity (approximately 50-60\%) and B-mode US is only used to guide prostate biopsies. There is a correlation between angiogenesis, as represented by microvascular density, and the presence of prostate cancer, its stage and survival [96]. Therefore, attempts have been made with contrast-enhanced col- or Doppler US to improve the detection and diagnosis of prostate cancer, with a reported increase in the detection rate of targeted biopsies of nearly $50 \%$ compared to systematic biopsies [97]. Low MI transrectal CEUS became available during the last decade when contrast-specific modalities were also implemented on endocavitary transducers, with further studies forthcoming [98 - 100].

\section{Study procedure}

Diagnostic CEUS is performed using transrectal US and typically a bolus of $2.4 \mathrm{~mL}$ of SonoVue ${ }^{\mathrm{TM}}$ (Bracco SpA, Milan) is administered to image particularly the inflow of UCA in a single plane. The most useful characteristics for an area suspicious for prostate cancer are a rapid inflow and/or an increased maximal enhancement compared to the surrounding tissue. Multiple UCA injections (typically four) are needed to image several planes [101]. CEUS has been used for follow-up of ablative treatments, with either a bolus injection or an infusion of UCA used to visualize perfusion defects resulting from the ablative therapy [102].

\section{Image interpretation and limitations}

Preliminary CEUS results appear to confirm the findings of contrast-enhanced Doppler US, with the lack of specificity of enhancing areas and of any other pattern suggesting cancer [98 - 100]. The evidence for the use of CEUS in the prostate remains limited and the role of CEUS in prostate cancer should still be considered a research subject. New improvements and new techniques are becoming available with the potential to increase the role of CEUS in prostate cancer detection and diagnosis. 4D contrastenhanced transrectal US imaging has now been introduced [103] and objective quantification techniques are being developed $[103,104]$. The first use of targeted UCA in humans was reported for prostate cancer; these VEGF-R2 targeted microbubbles were tested in a phase 0 trial in 24 patients (https://www.clinicaltrials. gov/ct2/show/NCT01253213?term=BR55\&rank=2?). The combination of CEUS and other US modalities such as elastography, in multi-parametric US could pave the way to a future clinically significant role for CEUS in prostate cancer detection and diagnosis [105].

\section{RECOMMENDATION 18}

Although CEUS for the improvement of the prostate cancer detection rate is an active research field, it currently cannot be recommended for clinical use (LoE 5, GoR C). Strong Consensus $(16 / 0 / 4,100 \%)$

\section{Transplanted Kidney}

All of the applications of CEUS in native kidneys also apply to renal transplants. B-mode and Doppler US are the modalities of choice for imaging transplanted kidneys but are limited in the assessment of microcirculation and the characterization of focal masses, inflammatory changes and complex cysts [27, 106, 107]. CEUS has a role in assessing vascular complications including arterial and venous thrombosis $[106,108,109]$. CEUS can image the 
microcirculation which is essential for assessing acute and chronic graft dysfunction, and is sensitive in the diagnosis of infarction, seen as a defect in all phases $[110,111]$. The defect on CEUS is smaller than on Doppler US, a manifestation of the imaging of smaller vessels on CEUS. Cortical infarction and ischemia (absent flow compared to hypoperfusion respectively) can be reliably differentiated on CEUS, a feature not possible by conventional Doppler US [112]. Different quantitative functional data have been assessed on time-intensity curves, all related to impaired parenchymal perfusion (e.g. longer time to peak, lower wash-in slopes, longer mean-transit time) and associated with a worse prognosis of graft function and survival [113-116]. Although these preliminary results are promising, further studies are needed to assess whether the detection of hemodynamic changes in renal grafts affects the management of patients with poorly functioning transplants. Consequently the quantification of CEUS is still considered a research field in transplant assessment.

\section{RECOMMENDATION 19}

CEUS can be used to identify renal transplant ischemia and vascular complications (LoE 3b, GoR B). Strong consensus $(20 / 0 / 0,100 \%)$

\section{RECOMMENDATION 20}

CEUS can be used to characterize complex cysts in renal transplant according to the Bosniak criteria (LoE 2b, GoR B). Strong Consensus (18/0/2, $100 \%)$

\section{RECOMMENDATION 21}

CEUS can be used to characterize indeterminate transplant renal lesions (LoE 2b, GoR B). Strong Consensus (19/0/1, $100 \%)$

\section{RECOMMENDATION 22}

CEUS can help evaluate patients with acute pyelonephritis (LoE 3a, GoR B). Strong Consensus (18/0/2, $100 \%$ )

\section{Adrenal Glands}

Conventional US is able to detect adrenal gland tumors [117], usually readily on the right side, but characterization is more difficult [118]. Size, irregular contours, inhomogeneity, loss of normal adrenal gland anatomy, and infiltration into adjacent organs, or the diaphragm, and vessels are criteria for malignancy. Malignant adrenal tumors may infiltrate and occlude the adrenal vein; the vascularity of a tumor thrombus may be demonstrated on CEUS. No CEUS criteria can reliably differentiate between benign and malignant adrenal gland tumors, with conflicting reports [119121]. Dynamic CEUS using time-intensity curve analysis has been deployed in the investigation of adrenal gland tumors without clear differentiation [120, 122]. CEUS may demonstrate characteristic hypervascularity of some adrenal gland tumors, e.g., pheochromocytoma, which typically also have necrotic regions with no contrast enhancement [121, 123, 124].

\section{RECOMMENDATION 23}

There is no evidence that CEUS can readily differentiate benign from malignant adrenal gland tumors (LoE 2b, GoR B). Strong consensus $(20 / 0 / 0,100 \%)$

\section{Obstetrics and Gynecology}

\section{Obstetrics}

The use of UCA in obstetrics is not indicated as there has been limited research related to the uncertainty of a possible underlying harmful effect. No recent human or animal studies have been performed. It is unknown whether the UCA passes through the placenta, though this seems unlikely as previously suggested [125, 126]. CEUS to assess a pregnant mother should be balanced against the risk of other imaging modalities.

\section{Gynecology}

\section{Uterus}

Both endometrial and cervical tumors have been assessed with CEUS [127, 128]. Perfusion differences between endometrial polyps and cancer have been documented [129], and CEUS during uterine artery embolization to treat leiomyomas might be useful $[130,131]$. Currently there is some benefit to the CEUS diagnosis of endometrial carcinoma [127]. No prospective trials have confirmed the value of CEUS for assessing uterine tumors and there is no proven clinical indication for CEUS use in the examination of the endometrium or the myometrium.

\section{Adnexa}

Differentiation of benign from malignant adnexal masses was attempted by visual assessment of UCA distribution and by quantification of enhanced Doppler signals, but, despite some difference in average values for some variables, no feature with sufficient clinical potential was obtained [132]. By using CEUS, it was demonstrated that adnexal masses without internal enhancement are invariably benign [133], but the presence of enhancement is not a specific sign of malignancy [128]. CEUS does not greatly improve the accuracy of color Doppler US for the diagnosis of malignancy in adnexal masses [134]. A multicenter study on the diagnosis of malignancy in adnexal masses, including quantitative CEUS features, confirmed that CEUS was not superior to conventional color Doppler US [135]. Although CEUS findings differed between benign and malignant ovarian masses, there was substantial overlap between benign and borderline tumors, although CEUS was able to differentiate invasive malignancies from other tumors [135]. 


\section{RECOMMENDATION 24}

There are no recommended gynecological clinical indications for the use of CEUS, despite the finding that the absence of any enhancement in adnexal masses corresponds to benign lesions (LoE 2b, GoR A). Strong consensus (19/1/0, $95 \%$ )

\section{Pancreas}

\section{Background}

CEUS is not indicated for the detection of focal solid or cystic pancreatic lesions, but CEUS improves the characterization of lesions seen on US [136-142].

\section{Study Procedure}

CEUS is superior to Doppler US techniques for the visualization of intrapancreatic vessels [142]. Enhancement begins immediately after aortic enhancement, with an arterial phase (10 to $30 \mathrm{~s}$ ), a venous phase (30 to approximately $120 \mathrm{~s}$ ) [2,4]. With a pancreatic mass, the CEUS examination also aims to characterize and confirm peripancreatic vascular associations [137-139, 143, 144]. The late venous phase begins about 120 seconds after the contrast injection and lasts for about 4 minutes. A late phase liver evaluation may identify possible metastatic lesions [3].

\section{Pancreatic Masses}

The enhancement pattern of focal pancreatic lesions is compared with the adjacent pancreatic tissue. The field of view should include both. This is mandatory with an isovascular mass but not essential with a hypovascular (hypoenhanced with few internal microbubbles) or hypervascular (hyperenhanced) mass [137]. CEUS provides clear distinction between vascularized solid lesions and cysts and provides information on lesions indeterminate on $\mathrm{CT}$, and may aid targeting areas following a first negative biopsy.

\section{Adenocarcinoma}

Ductal adenocarcinoma, the most common primary malignancy, is typically hypo-enhancing in all phases, because of the desmoplastic reaction with low vascular density that is present in $90 \%$ of cases [141, $145-150]$. Lesion size, margins and the relationship with peripancreatic vessels are better visualized with CEUS $[143,144]$. However, for assessing resectability, B-mode and color Doppler US are also adequate [137, 144]. CEUS is essential for lesion characterization $[140,151]$ and accurate liver staging $[3,137,152]$. CEUS can help with US-guided pancreatic biopsy $[153,154]$. Changes in pancreatic tumor vascularization during chemotherapy have been documented with CEUS $[155,156]$.

\section{Neuroendocrine tumors}

Neuroendocrine tumors typically present as hyper-enhancing lesions in the arterial phase of CEUS examinations, owing to their abundant arterialization, often not seen on color Doppler US [138, 157]. Necrotic avascular areas result in inhomogeneous enhance- ment in larger tumors [157, 158]. Based on the ENETs Consensus Guidelines, CEUS is reported as an imaging method for the diagnosis of neuroendocrine neoplasms [159].

\section{Mucin-producing cystic tumors}

CEUS improves the differentiation between pseudocysts and cystic tumors of the pancreas by accurately demonstrating vascularization of lesion septa or nodularity $[139,160,161]$. Mucinous cystadenoma is potentially malignant (may transform into cystadenocarcinoma), and it is usually depicted as an unilocular round cystic lesion, with particulate content, irregular thick walls, internal septa and parietal nodules which enhance on CEUS [139, 148, 160 - 164]. Intraductal papillary mucinous neoplasms (IPMN) are divided into main duct and side branch duct types. CEUS is helpful for differentiating between perfused (nodules) and non-perfused (mucin plugs) areas $[137,163]$. CEUS can be employed in the follow-up of borderline cystic lesions of the pancreas, if well visualized on US, in order to reduce the use of MR imaging [165].

\section{Serous cystadenoma}

Serous cystadenoma is a benign cystic lesion, typically with a lobulated microcystic appearance with thin and centrally oriented septa, which are vascularized on CEUS [139]. When the cysts are minute, microcystic serous cystadenomas may mimic a solid lesion, both on conventional US and CEUS, being hyperenhanced on CEUS [166]. Definitive differential diagnosis with respect to IPMN side branch duct types is not possible on CEUS. Exclusion of the presence of communication between the cystic lesion and the main pancreatic duct is required.

\section{Pseudocysts}

Pseudocysts typically contain non-vascularized debris, typically found in the early stages. Pseudocysts do not enhance at any phase with CEUS, even when heterogeneous on B-mode US [148, 162]. The reported sensitivity and specificity of CEUS in characterizing pseudocysts is up to $100 \%$ [160].

\section{Pancreatitis}

With acute pancreatitis, CEUS may delineate necrotic areas, which do not enhance $[167,168]$. If the pancreatic region is clearly visible on US, CEUS can be used in the follow-up of acute pancreatitis following CT staging, to reduce further CT examinations [167]. Good accuracy of CEUS for detecting necrotic lesions in acute pancreatitis (97.4\%) has been reported [168]. Significant correlation between CEUS and CT was found for the pancreatitis CT severity index, extent of necrosis and Balthazar grade, and as a predictor of severity in an episode of acute pancreatitis [167]. CEUS can be used as a follow-up imaging method in patients with initial CT staging at admission [167]. Focal mass-forming pancreatitis and autoimmune pancreatitis have been reported to have similar enhancement to that of the normal pancreatic parenchyma [145] and may be useful for the differentiation of pancreatic cancer $[140,169,170]$. 


\section{Pancreatic Transplant}

As with renal allografts, US is the modality of choice for imaging pancreatic transplants. CEUS can add extra value and diagnostic confidence when assessing graft perfusion and vascular complications such as arterial and venous thrombosis, particularly in complicated situations. CEUS can image the microcirculation to allow evaluation of viability and may provide prognostic information [171 - 173]. Early quantitative functional data shows promise in the diagnosis and management of rejection and represents a research field in transplant assessment [146, 174].

\section{RECOMMENDATION 25}

In solid pancreatic lesions detected on ultrasound, CEUS can be used to reliably characterize ductal adenocarcinoma (LOE 1a, GoR A). Broad consensus (18/0/2, $90 \%)$

\section{RECOMMENDATION 26}

CEUS can be used to distinguish between pancreatic ductal adenocarcinoma and neuroendocrine tumors (LoE 1a, GoR A). Strong consensus (20/0/0, $100 \%)$

\section{RECOMMENDATION 27}

CEUS can be used to differentiate between cystic neoplasms and pseudocysts (LoE 1a, GoR A). Strong consensus (20/0/0, $100 \%)$

\section{RECOMMENDATION 28}

CEUS can be used to differentiate vascular (solid) from avascular (e.g. liquid or necrotic) components of a pancreatic lesion (LoE 1b, GoR A). Strong consensus (20/0/0, 100\%)

\section{RECOMMENDATION 29}

CEUS can be used to define the dimensions and margins of a pancreatic lesion and its vascular relationships (LoE 2b, GoR A). Strong consensus (20/0/0, $100 \%)$

\section{RECOMMENDATION 30}

CEUS can be used to diagnose and follow-up acute necrotizing pancreatitis (LoE 1b, GoR A). Strong Consensus (19/0/1, $100 \%)$

\section{RECOMMENDATION 31}

CEUS can be used in the follow-up of indeterminate cystic pancreatic lesions (LoE 1b, GoR A). Strong consensus (20/0/ $0,100 \%)$

\section{RECOMMENDATION 32}

CEUS may improve the accuracy of percutaneous ultrasoundguided pancreatic procedures (LoE 2a, GoR B). Strong consensus $(20 / 0 / 0,100 \%)$

\section{RECOMMENDATION 33}

CEUS can be used to assess pancreatic graft ischemia and other vascular disorders (LoE 3b, GoR C). Strong consensus $(20 / 0 / 0,100 \%)$

\section{The Gastrointestinal Tract}

\section{Background}

Ultrasound imaging of the gastrointestinal ( $\mathrm{Gl}$ ) tract using $\geq 7.5 \mathrm{MHz}$ transducers usually reveals 5 wall layers and can identify a thickened bowel wall and focal lesions [175]. The imaging of the bowel wall with CEUS requires a higher UCA dose, typically $4.8 \mathrm{~mL}$ of SonoVue ${ }^{\mathrm{TM}}$, a consequence of fewer microbubbles of the appropriate size to resonate at higher frequencies [176]. The time of arrival of the UCA in the intestinal capillaries is usually 10 $20 \mathrm{~s}$ after injection, predominantly in the submucosal layer, with maximum concentration (peak intensity) reached after $30-40 \mathrm{~s}$. The arterial phase $(0-30 \mathrm{~s})$ is followed by a venous phase that lasts from $30-120 \mathrm{~s}$ [177].

\section{Study procedure}

The bowel should be examined in B-mode and Doppler US modes to detect the distribution of the relevant pathology, allowing the area of interest to be targeted for CEUS examination. A difference in perfusion between healthy and diseased bowel can be recognized by CEUS [178]. CEUS examination allows arterial and venous phases to be examined for two minutes and the possibility for a late phase liver examination for metastasis, if relevant.

\section{Inflammatory Bowel Disease (IBD)}

CEUS enables quantification of bowel wall vascularity in patients with Crohn's disease [179, 180] and is used to evaluate adult [181 - 183] and pediatric IBD patients [184]. CEUS correlates well with MR imaging of intestinal wall enhancement [185 - 187]. 


\section{Disease activity}

CEUS can add to B-mode and Doppler US in the evaluation of disease activity in IBD [188]. CEUS performs more reliably than power Doppler in estimating disease activity in Crohn's [180, 189]. Enhancement in different wall layers can be evaluated and quantified in Crohn's disease and correlates to a clinical activity index (CDAI) with good sensitivity and specificity [190, 191]. In ulcerative colitis, CEUS parameters correlate well with histological markers of inflammation [192]. Quantitative measurements of bowel enhancement obtained by CEUS also correlate with a severity grade determined at endoscopy [193]. Furthermore, histological markers of inflammation correlate well with CEUS perfusion [192, 194, 195]. Moreover, US evaluation of the changes of bowel wall enhancement during anti-inflammatory therapy may be useful for the clinical monitoring of Crohn's disease activity [195198]. CEUS can also be used to assess postoperative recurrence of Crohn's disease [199]. Two meta-analyses concluded that CEUS in the assessment of IBD activity is accurate and a highly sensitive and specific method [200, 201].

\section{Distinguishing between fibrous and inflammatory strictures}

In patients with a stricture of the bowel and resultant bowel obstruction, it is important to determine if there is active inflammation at the site of stricture or if this segment is fibrotic. Preliminary studies indicate that the use of UCA appears to be effective in the recognition of predominantly cicatricial stenosis in patients with Crohn's disease [202], although data is conflicting [203]. Using CEUS, the active inflammatory components will enhance, whereas the fibrotic stricture will not [21]. Absolute values for blood volume, flow and mean transit time of the bowel confirm that it is possible to distinguish between fibrous and inflammatory strictures in Crohn's disease [204].

\section{Abscesses}

Distinguishing abscesses from inflammatory infiltrates is an important clinical task in the management of Crohn's disease [205]. If areas of a significant size close to an affected bowel loop are completely devoid of UCA signals, this lesion represents an avascular abscess rather than inflammatory infiltrates [206, 207].

\section{Fistulas}

By injecting a UCA mixed with saline into one of the orifices of a fistula, it is possible to improve visualization of fistula routes in Crohn's disease, defining endocavitary and intraluminary locations [208-210]. Fistulas from blood vessels to intestines can also be detected using conventional intravenous CEUS [211].

\section{Intestinal Tumors}

US is not the imaging modality of choice for detecting intestinal polyps or tumors. Tumor vascularity can be evaluated by CEUS [212] and contrast enhancement of rectal cancer has been shown to correlate with histological vessel density [213]. Neuroendocrine tumors and gastrointestinal stromal tumors (GIST) of the stomach and small bowel are highly vascularized and CEUS can be applied for perfusion analysis and planning of US-guided biop- sies to avoid punctures of necrotic tumor parts [214]. Furthermore, the hypervascular ( $95 \%$ ) metastasis from neuroendocrine tumors to lymph nodes and the liver can be detected and characterized by CEUS [215].

\section{Transplanted bowel}

CEUS allows the detection of hypoperfusion of a bowel transplant graft [216]. As in other bowel diseases, CEUS can be used to evaluate the bowel wall perfusion as well as the patency of visceral vessels with the advantage of bedside examination. CEUS can also diagnose other organ complications after bowel transplantation, e. g. pancreatitis, when other imaging techniques cannot be performed. CEUS also allows diagnosis and monitoring of treatment response of intestinal acute graft versus host disease (I-aGVHD) after allografting. The detection of transmural penetration of the UCA into the bowel lumen indicates I-aGVHD [217, 218].

\section{Limitations}

It is difficult to visualize all bowel segments using transabdominal US. Intestinal peristalsis and luminal air will impair image quality and reduce the repeatability of the quantitative measurement of bowel enhancement patterns. Improved detection of intestinal inflammation may be enabled with targeted specific ligands attached to the UCA [219]. However, more studies are needed to establish the exact role of CEUS in the imaging of gastrointestinal pathology, and when performing multicenter studies, it is mandatory to standardize acquisition and software for quantification [220].

\section{RECOMMENDATION 34}

CEUS can be used to evaluate the vascularity of the gastrointestinal wall (LoE 1a, GoR A) and gastrointestinal tumors (LoE 4, GoR B). Broad Consensus (12/4/2, 75\%)

\section{RECOMMENDATION 35}

CEUS can be used to estimate disease activity in inflammatory bowel disease (LoE 1a, GoR A) and to discern between fibrous and inflammatory strictures in Crohn's disease (LoE 2b, GoR B). Strong consensus (19/1/0, 95\%)

\section{RECOMMENDATION 36}

CEUS can be used to monitor the effect of treatment in Crohn's disease (LoE 4, GoR B). Broad Consensus (17/1/2, $94 \%)$ 


\section{RECOMMENDATION 37}

CEUS can be used to detect abscesses (LoE 4, GoR C) and to confirm and track the route of fistulae (LoE 4, GoR C). Strong Consensus (19/0/1, $100 \%)$

\section{RECOMMENDATION 38}

CEUS can contribute to the evaluation of perfusion and vascular complications after intestinal transplantation (LoE 4, GoR C). Strong Consensus (18/0/2, $100 \%)$

\section{Spleen}

\section{Background}

Splenic abnormalities are uncommon [221] and frequently difficult to detect and characterize with conventional US. The spleen is ideally suited for CEUS due to its superficial location, homogeneous parenchyma, high vascularity, small size and long-lasting enhancement profile [222]. CEUS is a well-established technique for increasing diagnostic confidence and accuracy in splenic US.

\section{Study procedure}

Although UCAs remain entirely intravascular, they are sequestrated by the spleen [223], resulting in persistent late phase enhancement. Enhancement is inhomogeneous in the arterial phase ("zebra striped" pattern, similar to contrast-enhanced CT and MR imaging) [224, 225] but becomes homogeneous within $60 \mathrm{sec}-$ onds and usually persists for longer than 5 minutes. The arterial $(10-35 s)$ and late parenchymal phases ( $3-5$ min) are most valuable diagnostically. Scanning should be continuous during the arterial phase but then intermittent to avoid UCA destruction [226]. Enhancement of focal lesions is compared to adjacent (enhanced) splenic parenchyma. Deeper lesions can be obscured if a large volume of UCA is administered [222, 227 - 229]. 1.2 $2.4 \mathrm{~mL}$ of SonoVue ${ }^{\mathrm{TM}}$ is usually the optimal dose.

\section{Indications and image interpretation}

\section{Abnormal splenic size}

CEUS is not helpful in identifying the etiology of diffuse splenomegaly [224, 227]. Reduced or absent enhancement in a small spleen may indicate functional hypo/asplenia [230].

\section{Lesion identification}

Where the splenic parenchyma is inhomogeneous on B-mode US, the addition of CEUS will frequently demonstrate focal lesions [221, 222, 224, 230, 231].

\section{Ectopic splenic tissue}

Ectopic splenic tissue will enhance with the same pattern as the normal spleen. Late parenchymal enhancement will differentiate

splenunculi [227, 230, 232, 233] and splenosis [234] from pathological masses.

\section{Splenic infarction}

Infarction may be difficult to detect on conventional US, particularly when isoechoic in the acute stage. CEUS improves detection and characterization by demonstrating avascular, usually wedgeshaped, lesions [222, 224, 225, 230, 231, 235, 236]. Enhancement will be absent in patients with total splenic infarction [230]. CEUS can identify asymptomatic splenic infarction in patients with pancreatitis [237] and infective endocarditis [238].

\section{Characterization of focal splenic lesions (FSL)}

\section{Cystic lesions}

CEUS can be used in selected cases to show that complex cysts are avascular and therefore likely to be benign [225, 236]. Rim or septal enhancement may be a feature of splenic abscess formation [230, 236].

\section{Solid lesions}

B-mode and color Doppler US have low accuracy for the diagnosis of solid lesions. Small echogenic lesions are usually, but not always, benign, while echo-poor lesions are more likely to be malignant [222]. Correlation with the clinical history and laboratory tests is essential [221, 239-242]. Benign vascular tumors (BVT: hemangioma and hamartoma) are the most common benign lesions and secondary tumors (lymphoma and metastases) are the most common malignant lesions. No enhancement (in any phase) or persistent late phase enhancement is characteristic of benign lesions. Late phase washout is a feature of malignant lesions, but less pronounced washout is also seen in many benign lesions [221, 228, 229, 235, 236, 241]. Arterial phase hyper-/isoenhancement is an independent predictor of a BVT, more commonly seen in hemangiomas with an atypical appearance on conventional US [241, 242]. Nodular peripheral enhancement with progressive centripetal filling is unusual in splenic hemangiomas [222, 229, 241, 243]. Intralesional vessels, heterogeneous enhancement, necrotic regions and a dotted enhancement pattern favor a diagnosis of malignancy [227, 229, 244, 245].

Triage of patients with FSL

Lesions showing low-level arterial enhancement and progressive late-phase contrast washout usually require further imaging or biopsy, particularly in high-risk groups. FSL with benign enhancement characteristics will usually be suitable for interval imaging [229, 239, 242, 243].

\section{RECOMMENDATION 39}

CEUS may be used to improve the detection of focal splenic abnormalities (LoE 2b, GoR B). Strong Consensus (19/0/1, $100 \%)$ 


\section{RECOMMENDATION 40}

CEUS can be used to characterize suspected accessory spleens or splenosis (LoE 2b, GoR B). Strong consensus (20/0/0, $100 \%)$

\section{RECOMMENDATION 41}

CEUS can be used to diagnose splenic infarction (LoE 2b, GoR B). Strong consensus (20/0/0, 100\%)

\section{RECOMMENDATION 42}

CEUS can identify benign focal splenic lesions by showing persistent enhancement in the late phase (LoE 2b, GoR B). Strong Consensus (18/0/2, $100 \%)$

\section{Peripheral Vascular System and Aorta}

\section{Background}

The extracerebral vascular systems with indications for CEUS include the cervical carotid artery and the abdominal aorta, with less emphasis on peripheral arterial disease. Conventional US techniques are limited with respect to the demonstration of slow flow, especially in small vessels such as the vasa vasorum or collaterals and flow in critical stenosis, and the addition of a UCA may be useful.

\section{Study Procedures}

CEUS of the carotid and peripheral arteries is carried out with linear transducers $(5-10 \mathrm{MHz})$ and the abdominal aorta is visualized with convex transducers $(2.5-9 \mathrm{MHz})$. For diagnostic views of the vessels, 1.0 to $2.4 \mathrm{~mL}$ of SonoVue ${ }^{\mathrm{TM}}$ is intravenously administered as a bolus injection, followed by $10 \mathrm{~mL}$ of $0.9 \%$ normal saline solution.

\section{Carotid Artery}

\section{Stenosis}

Color and spectral Doppler US is the established imaging modality for suspected carotid artery disease. CEUS improves the sensitivity of Doppler US and can distinguish occlusion from tight subocclusive stenosis, comparable to contrast-enhanced CT angiography [246, 247]. CEUS improves the delineation of the endovascular border, characterizing the geometry of pre-stenotic, intra-stenotic and post-stenotic segments without the aliasing and blooming artifacts or angle dependence issues of Doppler US [248]. CEUS does not provide flow information [249].

\section{Follow-up after carotid stenting}

CEUS is a reliable method for evaluating re-stenosis after internal carotid artery stenting [250]. CEUS has fewer intrastenotic flow artifacts compared to Doppler US, resulting in improved visualization and depiction of the complete length and morphology of the stenosis [250].

\section{Dissection}

CEUS has been used to identify carotid dissection [251]. MR imaging remains the reference standard in the diagnosis of cervical vessel dissections. When it is contraindicated, the diagnostic accuracy of US examinations can be improved by the use of CEUS [248].

\section{Complications after vascular intervention}

Post-surgical fistula track visualization can be difficult using Doppler US but is improved with CEUS without artifacts [252]. Additionally, CEUS may help to image flow in false aneurysms with greater precision than Doppler US [248].

\section{Plaque characterization}

The accepted predictor of stroke risk is the degree of carotid stenosis, with contributing imaging features recognized [253, 254]. Plaque ulceration, which is a reliable marker of plaque vulnerability, can be clearly imaged using CEUS [255], which has superior sensitivity and diagnostic accuracy for the assessment of ulceration compared with conventional Doppler US [256]. Plaque neovascularization demonstrated by CEUS correlates well with histological findings [257-261], depicts inflammation as a marker of plaque vulnerability $[262,263]$, and may be used to predict cerebral ischemic events [255, 264 -269] and stratify risk for coronary artery disease $[270,271]$. The role of CEUS in routine clinical practice remains to be confirmed, particularly as objective assessment with quantification tools remains to be standardized [246].

\section{Large vessel vasculitides}

CEUS can also be used for the evaluation of large-vessel vasculitides, particularly to assess vascularization within the vessel wall. It improves the visualization of the lumen border, and allows dynamic assessment of carotid wall vascularization, which is a potential marker of disease activity [272, 273].

\section{Vertebral artery}

A hypoplastic vertebral artery is more frequently a risk factor for vertebrobasilar ischemia [274, 275]. A narrowed restricted artery (in the paired arteries) is more prone to closure, especially when other risk factors are present. Under difficult examining conditions, detection of low blood flow velocities in cases of hypoplasia can be difficult using conventional Doppler US. CEUS may differentiate between a hypoplastic vertebral artery and an occlusion at the origin. 


\section{Abdominal aortic}

CEUS can overcome some limitations of conventional US by improving the delineation of the aortic lumen and the detection of the main branching arteries. CEUS also improves the diagnosis of aortic rupture by detecting contrast extravasation [276].

\section{Aortic dissections}

A dissection of the abdominal aorta is usually an extension of a thoracic aortic dissection. In most cases, the true and false lumen can be discriminated with CEUS, because both early (true lumen) and late (false lumen) contrast enhancement can be detected, provided the false lumen is not thrombosed [277].

\section{Inflammatory Abdominal Aortic Aneurysm}

Inflammatory aneurysm of the abdominal aorta is a variant of atherosclerotic aneurysm that is characterized by inflammatory and/or fibrotic changes in the peri-aortic region of the retroperitoneum [278]. CEUS of the inflammatory aneurysm improves differentiation between covered rupture and inflammatory aortic aneurysm [279].

\section{Endovascular Aortic Graft Endoleak}

An endoleak, classified into subtypes depending on the site of the leak, represents blood flow outside the stent graft lumen but within the aneurysm sac and conventionally detected by CT angiography, although CT angiography is limited in the detection of some endoleak subtypes [280]. CEUS is able to identify and characterize an endoleak more accurately than CT angiography, with analysis of velocity and flow direction [281-285]. CEUS is particularly useful in the management of "endotension" often finding an occult endoleak, not seen on CT angiography [286]. CEUS enhancement quantification by time-intensity curves provides additional accuracy [287]. CEUS for the follow-up of patients with endoleak is possible [288, 289].

\section{Peripheral Vascular Disease}

CEUS has been used to improve the diagnostic capabilities of peripheral vascular US but has not demonstrated superiority [290]. Doppler US is the imaging modality of choice for the detection of complications after puncture of the femoral artery, with CEUS potentially improving diagnosis. The detection and localization of small lower leg arteries in patient with PVD requiring peripheral bypass graft surgery is improved with CEUS [291, 292].

\section{Limitations}

Limitations of CEUS exploration of the carotid artery and abdominal aorta relate to any conditions that prevent adequate US penetration and also limit conventional B-mode US exploration. Of particular regard are extensive wall calcification and subcutaneous emphysema after intervention or limited examination windows.

\section{RECOMMENDATION 43}

CEUS can help differentiate between total carotid and/or vertebral artery occlusion and residual flow in a tight stenosis (LoE 3, GoR B). Strong consensus (17/0/0, $100 \%)$

\section{RECOMMENDATION 44}

CEUS can be useful for the evaluation of carotid plaque neovascularization which suggests plaque instability (LoE $1 \mathrm{~b}$, GoR B). Strong consensus (20/0/0, $100 \%)$

\section{RECOMMENDATION 45}

CEUS can aid the identification of dissection of the extracranial carotid and vertebral arteries, as well as the abdominal aorta and its major branches (LoE 3, GoR C). Strong Consensus $(17 / 0 / 3,100 \%)$

\section{RECOMMENDATION 46}

CEUS may be an additional tool in the characterization of suspected inflammatory large vessel and abdominal aortic disease (LoE 5, GoR C). Strong Consensus (17/0/2, $100 \%)$

\section{RECOMMENDATION 47}

CEUS should be used in the follow-up of endovascular aortic repair (EVAR) for the detection and classification of endoleaks (LoE 1a, GoR A,). Strong consensus (20/0/0, $100 \%)$

\section{Cerebral Vessels}

\section{Background}

The major indication for CEUS in the examination of cerebral arteries is a poor signal with spectral Doppler US, preventing assessment of flow characteristics [293]. Contrast-enhanced transcranial color-coded duplex sonography (CE-TCCS) is best for simultaneously depicting B-mode brain anatomy as well as vessel detection and flow spectral Doppler tracing.

\section{Study procedures}

Transducers for CE-TCCS are identical to those for TCCS (sector $1.5-5.0 \mathrm{MHz}$ ). There are two applications using UCAs: main vessel, i. e. vascular imaging, and "perfusion imaging". For vascular imaging, UCAs are utilized to enhance Doppler signals in transtemporal or trans-nuchal transverse axial planes and coronal trans-temporal planes. Technical artifacts may cause inaccuracies: 1 ) bolus injection results in a blooming artifact preventing 
accurate Doppler spectral measurements. 2) UCA injection leads to an artificial increase $(1-36 \%)$ in maximum blood flow velocity, affecting stratification of a stenosis [294]. In perfusion imaging, either low or high MI CEUS is performed with trans-temporal insonation in the axial plane, but other insonation planes may also be used.

\section{Main intracerebral vessel imaging: interpretation and evaluation}

Vascular imaging

Most importantly, CE-TCCS is used to differentiate vessel occlusion in poor insonation conditions, and to detect very slow blood flow velocities and low flow volumes (small vessels, vessel pseudo-occlusion). The Doppler spectrum adds hemodynamic information to the anatomical information provided by color Doppler US.

\section{Examination of the anterior circulation}

A poor temporal bone window ( $45 \%$ in the elderly) can usually be overcome with CE-TCCS; over $85 \%$ of the basal arteries of the circle of Willis can be depicted satisfactorily after UCA administration [295]. CEUS infusion can be used in patients with poor acoustic windows for transcranial Doppler monitoring to test cerebral autoregulation as well as language lateralization for surgical planning.

\section{Examination of the posterior circulation}

CE-TCCS through the foramen magnum can increase the depth at which the intracranial vertebral arteries, the basilar artery, and the cerebellar artery segments can be identified and thus improve diagnostic confidence [294].

\section{Internal carotid artery stenosis}

Characterization of flow in the circle of Willis in patients with internal carotid artery stenosis and poor bone windows is important for estimating the risk of ipsilateral border zone infarction. Patients without collateral flow are particularly vulnerable to cross-clamping during carotid endarterectomy. The use of UCAs in these patients can provide valuable information for patient management [296].

\section{Stroke patients}

In acute stroke, the basal cerebral arteries can only be detected in $55-80 \%$ of cases with unenhanced TCCS. Reliable diagnoses can be obtained in $>85 \%$ with CE-TCCS with correlative findings on angiography in over $95 \%$ of cases [293].

\section{Perfusion imaging}

CEUS is performed with transtemporal insonation in the axial plane in perfusion imaging, although other insonation planes may be used. After administration of a UCA, the perfusion deficit can be detected according to the affected vascular territory in stroke patients, and in patients with space-occupying intracranial lesions [297 - 299]. CEUS perfusion imaging has been shown to improve prognostic assessment in the acute phase of cerebral ischemia and to provide comparable results to CT [300] and MR imaging [301]. After bolus injection, time-intensity curves can be generated to extract features that describe the perfusion characteristics quantitatively in standardized regions of interest [293, 302].

\section{Sono-thrombolysis}

The combination of systemic thrombolysis and repeated administration of a UCA over an hour in patients with middle cerebral artery occlusion accelerates recanalization but also increases hemorrhage into the infarct $[303,304]$. While of great interest as a method to enhance therapy, the hemorrhagic risk with repeated CEUS studies forced the two major sono-thrombolysis trials NOR-SASS and CLOTBUSTER to be terminated [305, 306].

\section{Limitations}

Despite UCA administration, only the proximal basilar artery can be evaluated. The distal portion can be depicted transtemporally, rendering the middle portion as a diagnostic gap for CE-TCCS. The quality of transtemporal unenhanced imaging is strongly predictive of the potential diagnostic benefit from the administration of a UCA. In patients without visible intracranial structures and vessels on conventional B-mode and Doppler US, there is little benefit from the addition of a UCA. The clinical value of the quantification of enhancement is limited by both physical and technical factors, with reliable identification of the absence of enhancement rather than the exact degree of blood supply impairment.

\section{RECOMMENDATION 48}

Contrast-enhanced transcranial Doppler/color duplex sonography (TCD/TCCS) improves the diagnostic capabilities of the examination (LoE 1b, GoR A). Strong Consensus (18/0/2, $100 \%)$

\section{Endoscopic}

\section{Contrast-Enhanced Endoscopic US (CE-EUS)}

\section{Background}

Contrast-enhanced endoscopic ultrasound (CE-EUS) combines the advantage of high-resolution US of the gastrointestinal wall, pancreas and other organs adjacent to the upper gastrointestinal tract with the ability to delineate micro- and macrovasculature $[307,308]$. Two different techniques are available: with contrastenhanced Doppler EUS (CED-EUS), the intensity of Doppler signals (color Doppler, power Doppler) is enhanced by the UCA, imaged at a high Ml, used to visualize slow, low-volume blood flow (e.g. tumor vessels). Disadvantages of this technique include artifacts caused by tissue motion and microbubble destruction. Contrastenhanced harmonic EUS (CEH-EUS) uses low MI techniques to visualize flow in small vessels and is established as an evidence- 
based technique [309] complementary to B-mode EUS and possibly EUS-elastography [310] to differentiate solid appearing structures, to characterize mass lesions, to improve staging of gastrointestinal and pancreatobiliary cancer and for real-time guidance of diagnostic and therapeutic EUS interventions [307 - 309, 311].

\section{Study procedures}

\section{Contrast-enhanced high mechanical index EUS}

In CED-EUS the color Doppler region of interest box should include the whole tumor where possible. CE endoscopic Doppler US scanning takes an extra 3-4 min. [312].

\section{Contrast-enhanced low mechanical index EUS}

"Peak-hold" techniques may be used to improve visualization with low $\mathrm{MI}$ techniques. With the high-frequency transducers used in EUS, a larger dose of UCA is used (e. g. $4.8 \mathrm{~mL}$ SonoVue ${ }^{\mathrm{TM}}$ ) [307, 308].

\section{Applications in pancreatic lesions}

\section{Differential diagnosis of solid pancreatic lesions}

CE-EUS can be used to differentiate between solid pancreatic lesions, mainly the hypoenhancing pancreatic ductal adenocarcinoma (PDAC) and iso- or hyper-enhancing solid pancreatic lesions (e. g. neuroendocrine tumors, pancreatic metastases, mass-forming focal pancreatitis, and serous microcystic cystadenoma). Meta-analyses showed a pooled sensitivity of $94 \%$ and a specificity of $89 \%$ for the differential diagnosis of PDAC from non-PDAC independent of the CE-EUS technique used [136, 313].

PDAC is characterized by hypoenhancement, irregular vascularization and a lack of venous vessels. Mass-forming focal pancreatitis exhibits netlike regular vascularization [314 - 317]. Hypovascularity was shown to have a high diagnostic value for the detection of solid pancreatic masses $\leq 20 \mathrm{~mm}$ [316], and for the differentiation of PDAC from inflammatory and non-PDAC neoplastic masses. For small pancreatic tumors $\leq 20 \mathrm{~mm}$, CED-EUS is significantly more accurate than CE-CT [316].

CEH-EUS is the more widely used technique for the differentiation of PDAC from other solid pancreatic lesions. With this technique, microvasculature and perfusion comparative qualitative or quantitative (time-intensity curves, TIC) analysis may be performed. In general, heterogeneous hypoenhancement is typical for PDAC, whereas almost all solid non-PDAC lesions exhibit isoor hyperenhancement [313, 318-322]. Hyperenhancement with slow washout is a typical pattern of pancreatic neuroendocrine tumors (P-NET), while filling defects are highly predictive of malignancy [322 - 326]. In pseudo-tumoral chronic pancreatitis, isoenhancement and hyperenhancement are the most common patterns [319, 321-323, 327, 328]. Both focal and diffuse autoimmune pancreatitis demonstrate hyper-enhancement with CEH-EUS [329]. Prospective studies indicate that the diagnostic accuracy of CEH-EUS and EUS-FNA is comparable [320, 321, 323]. An accurate differential diagnosis of small solid pancreatic lesions is important. A retrospective multicenter study showed a high accuracy ( $86 \%$ ) of CEH-EUS for the differentiation of small solid pancreatic lesions $\leq 15 \mathrm{~mm}$ [330]. Concomitant use of both EUS-FNA and CEH-EUS increases the diagnostic yield and accuracy of EUS-FNA [323, 331, 332]. In patients with hypoenhancing solid pancreatic lesions and negative EUS-FNA, continuing suspicion of PDAC demands repeat tissue sampling.

\section{Staging in pancreatic ductal adenocarcinoma}

CEH-EUS may increase the accuracy of preoperative tumor staging and resectability evaluation of pancreatico-biliary malignancies $[327,333]$.

\section{Characterization of cystic pancreatic lesions}

Contrast enhancement of the wall, septations, nodules or solid parts of cystic pancreatic lesions in CE-EUS reliably separates neoplastic pancreatic cysts from pseudocysts and non-neoplastic cysts [334 - 336]. However, differentiation between serous cystadenoma and mucinous neoplastic cysts is not improved by CE-EUS [336, 337]. CE-EUS is significantly more reliable for the differentiation of mural nodules from intracystic mucus or debris than contrast-enhanced CT and B-mode EUS [336 - 340]. Further characterization of mural nodules by CE-EUS (morphological type, height, degree of enhancement) has been shown to be useful for risk stratification [337, 341].

Applications in non-pancreatic lesions

\section{Gallbladder lesions}

CEH-EUS improves the diagnosis of malignant gallbladder polyps and wall thickening over B-mode EUS [342 - 345]. In gallbladder malignancy (protruding lesions as well as circumferential wall thickening), a heterogeneous enhancement pattern, the presence of perfusion defects and an irregular vessel pattern were found to be typical features with CEH-EUS. Homogeneous or absent enhancement occurs with benign lesions [342 - 345].

\section{Characterization of lymph nodes}

Heterogeneous lymph node enhancement with focal filling defects is present in lymph nodes with metastatic infiltration, whereas the majority of benign lymph nodes demonstrate homogeneous enhancement [346]. However, due to similar homogeneous enhancement patterns of both benign lymph nodes and malignant lymphoma, the sensitivity was not improved over B-mode US [347]. CEH-EUS had a similar accuracy as EUS-FNA for diagnosing lymph node metastasis of pancreatobiliary cancer [348].

\section{Gastrointestinal wall lesions}

Assessment of the vascularization of gastric cancer is feasible using CE-EUS [349, 350]. The intensity of enhancement is correlated with pathological criteria of neo-angiogenesis (microvascular density; vascular endothelial growth factor, VGEF) [349]. CEH-EUS allows the assessment of treatment-induced changes of tumor vascularity in gastric cancer [350]. Differentiation between potentially malignant GIST and benign sub-epithelial tumors of the upper gastrointestinal tract can be improved by CE-EUS [351, 
352]. Significantly stronger enhancement was observed in GIST than in leiomyoma and lipoma [353]. The detection of irregular intratumoral vessels in the arterial phase and a heterogeneous enhancement pattern are highly predictive for intermediate or high-risk GIST [353-355].

\section{Visceral vascular diseases}

CE-EUS may be used to diagnose splanchnic arterial and venous occlusive disease [356]. Moreover, CE-EUS improves the visualization of flow in esophageal varices, paraesophageal veins and perforating veins [357-359].

\section{RECOMMENDATION 49}

Both low and high mechanical index (MI) contrast-enhanced (CE)-EUS techniques can help the characterization of solid pancreatic lesions (LoE 2a; GoR B), especially low MI CE-EUS for small ( $\leq 20 \mathrm{~mm}$ ) lesions (LoE $1 \mathrm{~b}$; GoR A). Strong consensus (20/0/0, 100\%)

\section{RECOMMENDATION 50}

CE-EUS can be used to distinguish between pancreatic ductal adenocarcinoma and neuroendocrine tumors (LoE 1b, GoR A). Strong Consensus (19/0/1, 100\%)

\section{RECOMMENDATION 51}

CE-EUS can be used to guide and target EUS-FNA of pancreatic lesions (LoE 2b, GoR C). Strong consensus (20/0/0, $100 \%$ )

\section{RECOMMENDATION 52}

CE-EUS allows accurate differentiation of cystic pancreatic neoplasms from pancreatic pseudocysts (LoE 2b, GoR B). Strong consensus $(20 / 0 / 0,100 \%)$

\section{Abdominal Trauma}

\section{Background}

CT imaging remains the standard technique for evaluating hemodynamically stable patients with high-energy multi-trauma, allowing rapid triage and reducing morbidity and mortality. CT has inherent disadvantages, which restrict its adequacy in some clinical scenarios: the patient needs to be stable and cooperative (sedation may be required, particularly in pediatric patients), it utilizes iodinated contrast media and carries the risks associated with radiation exposure. The latter is an important limiting factor, especially when a low-risk mechanism of injury and the patient's condition would not necessarily warrant a CT examination, even though an imaging investigation is required. Focused Assessment with Sonography for Trauma (FAST) has been widely used in the diagnostic pathway of the trauma patient, with a sensitivity that ranges from $63 \%$ to $99 \%$ for the detection of free fluid [360], but has poor sensitivity in the diagnosis of parenchymal injuries. UCAs have significantly improved the diagnostic performance of B-mode US in the depiction of solid organ injuries [361-363], with performance close to that of CT: CEUS can achieve a sensitivity and specificity of $99 \%$ [364], avoiding overutilization of CT [365].

\section{Study procedure}

Commencing from the side of clinical concern, the examination should begin with the kidneys, their enhancement being the most fleeting, followed by the adrenals, liver, pancreas and lastly the spleen. The kidneys are studied during the arterial phase, while the liver, spleen, pancreas and adrenals are studied in the venous phases. Most commonly, the CEUS examination utilizes two separate doses of intravenous UCA: one dose is used to evaluate the right kidney, right adrenal, liver and pancreas and the second dose is aimed at the left kidney, left adrenal and spleen. In follow-up CEUS examinations, the known injured organ is targeted.

\section{Image interpretation of abdominal injuries}

On CEUS, lacerations and hematomas will show a complete lack of enhancement and will be clearly demarcated against the normal parenchyma, whereas areas of contusion may demonstrate faint enhancement. CEUS may also readily depict areas of hyperemia, infarct and active bleeding: any UCA pooling in the abdominal cavity will indicate the presence of ongoing hemorrhage, management becomes interventional. Crucial complications such as devascularized parenchyma, pseudoaneurysm formation and acute cortical necrosis of the kidney can also be promptly diagnosed on CEUS [41, 366]. CEUS can also allow further evaluation of abdominal injuries where CT findings are uncertain due to artifacts or where CT is discouraged, e. g. in renal impairment and in children. CEUS can confidently exclude major abdominal visceral injuries and therefore, patients sustaining minor, low-energy trauma can be discharged following a normal CEUS examination without the need to perform a CT examination.

\section{Limitations}

CEUS cannot diagnose traumatic lesions of the pelvicalyceal system, UCAs being purely intravascular and not excreted through the renal collecting system. The limitations with respect to bowel injury and a hemodynamically unstable patient would warrant a CT examination rather than a CEUS examination.

\section{RECOMMENDATION 53}

CEUS can be used in hemodynamically stable patients with isolated blunt moderate-energy abdominal trauma to evaluate solid organ injury as an alternative to $\mathrm{CT}$, particularly in children (LoE 1b, GoR A). Strong consensus (20/0/0, $100 \%$ ) 


\section{RECOMMENDATION 54}

CEUS can be used to further evaluate uncertain CT findings related to abdominal trauma (LoE $2 b, G o R C)$. Strong consensus $(20 / 0 / 0,100 \%)$

\section{RECOMMENDATION 55}

CEUS can be used in the follow-up of conservatively managed abdominal trauma to reduce the number of CT examinations, particularly in children (LoE 1b, GoR B). Strong consensus (20/0/0, 100\%)

\section{Superficial Structures}

\section{Thyroid}

\section{Background}

An increasing thyroid malignancy incidence $(\leq 8$ per 100000 in Europe) [367] would benefit from a noninvasive diagnostic method that allows reliable differentiation between malignant and benign thyroid nodules, superior to the current B-mode US features [368]. CEUS is able to focus on the analysis of macroand microvascularization patterns [369-371].

\section{Study procedure}

\section{Thyroid nodule assessment}

\section{Qualitative parameters}

Qualitative parameters characterize nodule vascularization compared to the surrounding tissue, defined as intensity, homogeneity, UCA uptake and washout rate [369 - 371]. Hypoenhancement is the most precise predictor of malignancy on CEUS with high sensitivity, specificity and accuracy of $82 \%, 85 \%$ and $84 \%$ respectively [372]. A heterogeneous contrast enhancement pattern has a sensitivity, specificity and accuracy of $88.2 \%, 92.5 \%$ and $90.4 \%$, respectively [370, $373-376]$. A ring enhancement pattern of a solid thyroid nodule is likely a benign feature with a sensitivity, specificity and accuracy of $83.0 \%, 94.1 \%$ and $88.5 \%$, respectively [370, 374].

\section{Quantitative parameters}

Time-intensity curves (TIC) for quantitative analysis are generated by placing the region of interest (ROI) in the nodule occupying the largest possible nodule area and comparing it to the surrounding tissues. The following parameters are evaluated: area of ROI, wash-in slope, time to peak, peak intensity, area under the curve, mean transit time and washout $[369,373]$. Time-intensity curve patterns of washout may appear as poly-phasic or mono-phasic related to the heterogeneity of the nodule and histology, with a sensitivity, specificity and accuracy of $76.9 \%, 84.8 \%$ and $82.6 \%$, respectively [373, 375, 376].

\section{Size}

Nodule size affects CEUS examination and interpretation. A nodule $<10 \mathrm{~mm}$ shows absent vascularization (incomplete neovascularization), while larger nodules $>10 \mathrm{~mm}$ appear hypervascular [369, 371, 375].

\section{Image interpretation}

The diagnostic value of CEUS has been analyzed in a meta-analysis showing high pooled accuracy of CEUS in the differentiation between benign and malignant nodules [376, $376-380$ ]. There is insufficient evidence regarding the application of CEUS in the assessment of thyroiditis.

\section{Limitations}

CEUS is a promising noninvasive method for the differential diagnosis of benign and malignant thyroid nodules. However, overlapping data between CEUS qualitative and quantitative evaluation parameters and criteria of benign and malignant nodules indicate a limitation in the interpretation of tumor microvascularity. No single indicator is sufficiently sensitive or specific. Therefore, the results should be interpreted in conjunction with the clinical data, conventional US and other imaging examination findings to improve diagnostic accuracy in the assessment of thyroid nodules [371, 372, 375].

\section{RECOMMENDATION 56}

CEUS for the characterization of thyroid nodules is an active research field but at present cannot be recommended for clinical use (LoE 2b, GoR C). Strong Consensus (19/0/1, $100 \%$ )

\section{Lymph Nodes}

\section{Background}

The US discrimination between benign and malignant superficial lymphadenopathy is dependent on shape, anatomical appearance and vascular pattern with a wide range of sensitivities and specificities [381, 382]. UCAs have been shown to increase the accuracy of the analysis of the vascular pattern using the conventional color Doppler mode [383].

\section{Study procedure}

Normal linear high-frequency transducers enabled for CEUS examination with higher doses of UCA administered, normally $4.8 \mathrm{~mL}$ of SonoVue ${ }^{\mathrm{TM}}$, as in other superficial structures, are used.

\section{Image interpretation}

Malignant neo-vascularization, demonstrated when vessels penetrate the capsule of the node away from the hilum, is a characteristic feature of a metastatic lymph node [381, 382]. A benign reactive lymph node has preserved morphology and vascular anatomy, with a single vascular pedicle at the hilum, containing both arteries and veins, regularly branching towards the periphery of the lymph node [381, 382]. Display of the vascular anatomy can 
be facilitated by the addition of UCA during color Doppler US [383 - 385]. Using low MI CEUS techniques further improves characterization with a sensitivity and specificity of $93 \%$ and $88 \%$, respectively [384, $386-389]$.

US studies of lymph node vascularization have limitations, with most studies undertaken in specialized units and in patients with known head and neck cancer, melanoma or breast cancer [390]. Vessel distribution analysis is usually satisfactory if the whole lymph node is involved but is less informative when focal lymph node involvement or necrosis renders a CEUS examination (as well as other imaging modalities) inconclusive. Lymph nodes with lymphoma infiltration are unique as the vascular pattern resembles that of non-malignant nodes [390, 391], with a reported "speckled" pattern [392] and a different configuration on timeintensity curves [393] that may help to improve diagnosis.

\section{RECOMMENDATION 57}

CEUS for the characterization of superficial lymphadenopathy is an active research field but at present cannot be recommended for clinical use (LoE 2b, GoR C). Strong consensus $(20 / 0 / 0,100 \%)$

\section{Salivary Glands}

\section{Background}

The B-mode US, Doppler US, elastography and CEUS appearance of parotid gland lesions has been analyzed to discriminate benign from malignant lesions [394-396]. CEUS investigations were initially performed with high MI US using color Doppler [394] and later with low MI techniques [397, 398]. The role of CEUS assessment of parotid gland lesions is limited by a paucity of studies, and relies on expert opinion [399, 400].

\section{Study procedure}

Normal linear high-frequency transducers enabled for CEUS examination with higher doses of UCA administered, normally $4.8 \mathrm{~mL}$ of SonoVue ${ }^{\mathrm{TM}}$, as in other superficial structures, are used, but fractionated doses have also been used [396].

\section{Image interpretation}

\section{Qualitative approach}

Malignant lesions demonstrate chaotic vessel formation on color Doppler US with increased enhancement with CEUS or with prominent organized vessel formation combined with slight enhancement on CEUS. Monomorphic adenomas showed vascularization patterns of all other entities [396]. Pleomorphic adenomas with minor vessel identification on color Doppler US result in poor perfusion on CEUS. All Warthin's tumors show prominent internal vessel visualization on color Doppler US and increased enhancement with CEUS. According to the morphologic and distribution features of microvascularity, CEUS imaging of the lesions can be classified into three types: diffuse homogeneous enhancement (type 1), heterogeneous enhancement (type 2) and no enhancement/iso-enhancement (type 3). Types 1 and 3 are suggestive of benign tumors; Type 2 can indicate the presence of a malignant lesion [397].

\section{Quantitative approach}

Parotid gland lesions can be divided into different benign and malignant lesions by using specific time-intensity curve parameters from CEUS measurements. Malignant lesions appear highly vascularized, while benign lesions enhance less [398, 401]. The area under curve (AUC) and mean transit time (MTT) show significantly higher values for malignant lesions. The intratumoral time to peak in pleomorphic adenoma appears markedly longer than in cystadenolymphoma [402].

\section{RECOMMENDATION 58}

CEUS for the characterization of salivary gland lesions cannot be recommended for clinical use (LoE 2b, GoR C). Strong consensus $(20 / 0 / 0,100 \%)$

\section{Breast}

\section{Background}

CEUS in the differential diagnosis of breast masses was an early application, with encouraging initial results, but this early promise has not been fulfilled, despite numerous studies using modern methods including temporal accumulation methods (microvascular imaging) [403-405]. Studies relating to CEUS, MR imaging and biological factors [406] and studies to obtain a precise qualitative and quantitative vascular map of the tumor, which appears to correlate well with prognostic factors [407] or use CEUS to identify BI-RADS category 3 or 4 small breast lesions [408] have all been conducted, without specific patterns of CEUS enhancement of malignant lesions. A single study documented characteristic CEUS enhancement patterns which could be helpful for identifying papillary lesions and for predicting a potentially malignant papilloma [409]. CEUS has been used to estimate tumor size in invasive ductal cancer, which in turn predicted regional lymph node metastasis [410]. Notwithstanding, no specific pattern indicating malignancy has been identified and, although an important research topic, cannot be recommended for routine clinical use.

\section{RECOMMENDATION 59}

CEUS for the characterization of breast lesions is an active research field but at present cannot be recommended for clinical use (LoE 2b, GoR C). Strong consensus (20/0/0, 100\%)

\section{Sentinel lymph nodes}

CEUS can be used for detecting axillary sentinel lymph nodes in cancer patients. SonoVue ${ }^{\mathrm{TM}} 1 \mathrm{~mL}$ (or $2 \mathrm{~mL}$ Sonazoid ${ }^{\mathrm{TM}}$ [411]) as the UCA 
is injected intradermally into the locally anesthetized periareolar skin towards the upper outer quadrant of the involved breast. The UCA is taken up by the subdermal lymphatics and the enhanced lymphatics can be traced to the sentinel node(s) [412-418]. Initial experience indicates that the method is non-toxic and performs as well as the blue dye or radioisotope methods [414]. It enables core biopsy of the sentinel node and, if positive on histology, is a reliable indicator of nodal involvement, directing patient counselling.

\section{RECOMMENDATION 60}

CEUS with intradermal injection of contrast agent to identify the sentinel lymph node is an active research field but at present cannot be recommended for clinical use (LoE 2b, GoR C). Strong consensus $(20 / 0 / 0,100 \%)$

\section{Inflammatory joint diseases}

\section{Background}

Color or power Doppler US can detect the vascularity in the synovial proliferation associated with inflammatory activity. However, these US techniques have limited sensitivity and could benefit from the addition of a UCA.

\section{Study procedure}

Reports on the use of UCAs in inflammatory joint disease detect enhancement by conventional color or power Doppler US (CE Doppler). A full dose of $4.8 \mathrm{~mL}$ (SonoVue ${ }^{\mathrm{TM}}$ ) is used with standard transducers and equipment when investigating joints with CEUS.

\section{Image interpretation}

\section{Arthritis and synovitis}

Microscopic examination of synovial biopsies shows angiogenesis from the earliest stages of inflammatory disease. Proliferation of hypervascularized pannus can be detected before joint destruction. It correlates with disease activity and appears to be crucial to its invasive and destructive behavior [419]. The development of novel biological therapies (e. g. tumor necrosis factor-alpha inhibitors), which target the microvasculature, needs more sensitive vascular imaging to assess response to treatment [419-423]. The addition of UCAs to Doppler US significantly improves the detection of vascularity in active rheumatoid arthritis [424, 425] and psoriatic arthritis [426]. There is also evidence that CE power Doppler US helps to differentiate active from inactive disease in subclinical juvenile rheumatoid arthritis of the knee [427]. CE Doppler correlates with the findings of CEMR imaging, indicating the degree of inflammation in patients with synovitis [427]. CE Doppler is also more useful in the diagnosis of sacroiliitis than conventional Doppler [428, 429].

\section{Differentiation between synovial pannus and fluid}

Early detection of vascularized synovia is a primary goal of the assessment of inflammation. CE Doppler US improves the differentiation between active synovitis and other articular thickenings, such as fibrotic pannus and articular fluid [430 - 432].

\section{Bursae and tendon}

Contrast administration can highlight peripheral enhancement on Doppler US, corresponding to the vascularized synovial lining of an inflamed bursa, and can better differentiate between fluid, fibrous and hypervascular synovial thickening in comparison to non-enhanced Doppler US [433-435].

\section{Therapeutic follow-up}

Successful treatment results in a decrease in synovial thickening and necrosis of the pannus with reduction of vascularity and Doppler US signals. The distinction between fibrous pannus and active synovial proliferation is important during follow-up, because the volume of the synovium itself is not clinically significant, as it may contain varying amounts of fibrous tissue. Fibrotic pannus shows no vascularity on conventional power Doppler US and lacks enhancement on CEUS [436-440].

\section{RECOMMENDATION 61}

CEUS for the further assessment of the degree of vascularization and for treatment monitoring in joints is an active research field but at present cannot be recommended for clinical use (LoE 2b, GoR C). Strong Consensus (18/0/2, $100 \%$ )

\section{Gallbladder diseases}

\section{Background}

B-mode and color Doppler US are the first-line imaging modalities for the diagnosis of gallbladder disease. The use of CEUS improves the diagnostic accuracy of US in selected cases. CEUS is not indicated if conventional US provides a clear diagnosis.

\section{Study Procedure}

For evaluation of the gallbladder wall, $1.2-2.4 \mathrm{~mL}$ of SonoVue ${ }^{\mathrm{TM}}$ is used, unless a high-frequency transducer is used when the dose is increased to $4.8 \mathrm{~mL}$ [176], with the arterial phase (<30 s) differentiated from the venous phase (>31s) [441-445]. CEUS study of the gallbladder wall evaluates perfusion, contrast kinetics, branching intramural vessels and gallbladder wall integrity; with a late liver sweep for malignant metastasis.

\section{Cholecystitis}

Acute cholecystitis is normally associated with cholelithiasis, acalculous cholecystitis accounts for the minority of cases, but is associated with a higher incidence of gangrene and perforation [446]. In acute cholecystitis, the inflammatory process may involve the 
adjacent liver tissue ("reactive hepatitis" [447]) causing hepatic arterial hyperenhancement on CEUS. Gangrenous cholecystitis, transmural necrosis of the gallbladder wall, causes a discontinuous or irregular gallbladder on CEUS [448, 449]. CEUS should be considered in patients at risk for complicated acute cholecystitis [442, 450 - 453]. Superficial or infiltrating gallbladder carcinoma may mimic chronic cholecystitis, presenting with diffuse wall thickening, with stones or sludge obscuring a malignant tumor. CEUS may help to detect a silent carcinoma [445, 454, 455].

\section{Tumors of the gallbladder wall}

\section{Polypoid lesions}

Polypoid gallbladder lesions are commonly seen on US $(2.6 \%$ $-12.1 \%$ of cholecystectomy specimens) [456]. In primary sclerosing cholangitis and gastrointestinal polyposis syndromes, $60 \%$ of gallbladder polyps are malignant [457]. Malignancy in gallbladder polyps between $6-10 \mathrm{~mm}$ is extremely rare, while polyps $>10 \mathrm{~mm}$ are regarded as preinvasive adenomas and papillary neoplasms [456, 458]. Adenomas have a wider vascular stalk, thought to be significant, that is best seen on CEUS [456, 459]. It remains unclear if CEUS can contribute to the differentiation between polyps, adenomas and noninvasive gallbladder carcinoma [441, 460]. Polyps $>10 \mathrm{~mm}$ which show an iso- and inhomogeneous enhancement pattern may be a criterion to differentiate adenomas from cholesterol polyps.

\section{Adenomyomatosis}

Adenomyomatosis is a hyperplastic process of the gallbladder wall affecting the complete gallbladder wall or parts of it, with the fundus representing the most frequent site. No intrinsic malignant potential has been described, but has been reported at $6.6 \%$ in Asia [461]. On CEUS, the thickened wall demonstrates isoenhancement with a small non-enhancement rim surrounding the gallbladder [444, 462].

\section{Adenocarcinoma of the wall}

Adenocarcinoma is the most common malignancy of the gallbladder arising in the majority of cases from underlying chronic cholecystitis [463]. Nonspecific clinical signs result in a late diagnosis with a 5-year survival rate of $5 \%$ [464]. Differentiation between benign and malignant gallbladder tumors cannot be made by hyperenhancement during the arterial phase as gallbladder cancers (85\%) and benign gallbladder diseases (70\%) both show hyperenhancement $[452,455]$. The CEUS features of washout within $35 \mathrm{~s}$ after UCA administration, the destruction of gallbladder wall integrity and infiltration of the adjacent liver tissue are highly suggestive features of malignancy and highly suggestive of gallbladder cancer $[441,445]$. CEUS can be used to differentiate between GB tumors and biliary sludge [465]. Gallbladder wall destruction beneath a solid lesion and the infiltration of adjacent liver tissue are highly suggestive features of malignancy [441, 444, 445, 460, 461, 465].

\section{Gallbladder metastasis}

Metastatic lesions of the gallbladder wall are rare with melanoma accounting for $>50 \%$ [466]. On B-mode and CEUS, exophytic mural tumor nodules extend into the lumen of the gallbladder, with CEUS indicating a flow away from the wall [467].

\section{RECOMMENDATION 62}

CEUS can be used in acute cholecystitis to better detect local complications (LoE 2b, GoR B). Strong Consensus (19/0/1, $100 \%)$

\section{RECOMMENDATION 63}

CEUS may differentiate chronic cholecystitis from gallbladder carcinoma (LoE 2b, GoR B). Strong Consensus (19/0/1, $100 \%)$

\section{RECOMMENDATION 64}

CEUS is able to differentiate between a perfused gallbladder lesion and motionless biliary sludge (LoE 4, GoR C). Strong consensus $(20 / 0 / 0,100 \%)$

\section{Neurosurgery}

\section{Background}

Intraoperative CEUS (iCEUS) allows for excellent evaluation with distinct enhancement phases and good delineation between lesions/vessels and healthy structures. These iCEUS features, together with high temporal and spatial resolution, make iCEUS invaluable in neurosurgery for vascular and oncological applications [468 - 474].

\section{Study procedure}

\section{Ultrasound equipment}

A contrast-enabled multi-frequency linear array transducer (3$11 \mathrm{MHz}$ ), most frequently using the UCA SonoVue ${ }^{\mathrm{TM}}$, is deployed [468-475].

\section{Examination technique}

Patient positioning and craniotomy must be designed to allow direct contact of the transducer with the brain surface or the cavity filled with saline, to allow transducer manipulation. A preliminary CEUS examination is performed through the dura-mater after bone flap removal, preceded by a B-mode US examination, allowing identification of anatomical landmarks and lesion position/relationships. A standard evaluation to identify principal arteries, capillary and veins in the region of interest, evaluating the timing distribution and degree of UCA enhancement, is required [468 -475]. 


\section{Intraoperative applications}

Intraoperative evaluation of cerebral and spinal neoplastic lesions

\section{Neoplastic lesion identification}

Standard B-mode US is able to visualize and delineate most neoplastic lesions, both intra- and extra-axial. In intrinsic tumors with ill-defined borders or in the presence of brain edema, B-mode US is insufficient to evaluate tumor morphology and borders. iCEUS highlights the tumor parenchyma and the tumor-brain interface accurately, relying on the abnormal density of capillaries between the pathological tissue and the surrounding parenchyma [161, 468, 469, 471, 472, 474, 476, 477].

\section{Tumor characterization}

The degree of contrast enhancement and distribution is related to the density of capillaries in the region of interest. iCEUS allows real-time characterization of different histological types and grades dependent on timing, distribution and degree of contrast enhancement [468, 469, 474, 478, 479].

\section{Tumor vascularization and surgical strategy}

The direct visualization of parental and surrounding vessels allows determination of vessel location in the surgical field and optimizes the surgical strategy, allowing for early tumor devascularization before removal, thus reducing intraoperative bleeding [468, $470-472,478,480]$.

\section{Tumor resection control}

Safe repeated iCEUS examinations during surgery allow visualization of residual tumor within the surgical cavity, overcoming B-mode US limitations. iCEUS also allows assessment of complete tumor removal, showing no remaining enhancing areas and an absence of abnormal venous drainage [472, 477].

\section{Intraoperative angio-sonography for cerebral and spinal} vascular lesions

Vascular malformations are identified with Doppler US. iCEUS allows interpretation of the vascular tree prior to surgical exposure. iCEUS is able to determine the location of a vessel and follow its entire course and may be used with many different vascular abnormalities. iCEUS can also verify exclusion of the lesion from the circulation at the end of the procedure, and can also asses flow direction, vessel patency after aneurysm clipping, and brain perfusion in the distal territories $[470,473,480]$.

\section{Intraoperative contrast-enhanced ultrasound in traumatic} brain surgery

iCEUS during surgery for trauma allows distinction between normal and injured brain tissue, more clearly than B-mode and color Doppler US. This improves the accuracy of the classification of traumatic brain injury, effectively removing hematoma and/or infarcted brain while preserving healthy tissue [475].

\section{Limitations}

Operator training is paramount and the craniotomy has to be large enough to allow free transducer movement. Transducer pressure on neural structures must not damage vessels or the parenchyma. Static retractors must be temporarily removed during iCEUS evaluation. The use of hemostatic materials must be limited to prevent artifact formation and restriction of the field of view.

\section{RECOMMENDATION 65}

Intraoperative CEUS is indicated in neuro-oncological procedures for tumor identification, assessment of boundaries, perfusion pattern and evaluation of residual tumor (LoE 4, GoR C). Strong consensus (19/0/0, $100 \%)$

\section{RECOMMENDATION 66}

Intraoperative CEUS is indicated in angiosonography for neurovascular procedures ( $L O E$ 4, GoR C). Strong consensus $(19 / 0 / 0,100 \%)$

\section{RECOMMENDATION 67}

Intraoperative CEUS is indicated in traumatic brain surgery to demonstrate tissue viability (LoE 4, GoR C). Strong Consensus $(18 / 0 / 1,100 \%)$

\section{Interventional CEUS}

\section{Background}

CEUS has obvious potential during US-guided interventional procedures. Any CEUS-guided intervention can be performed in a similar manner to the routine US-guided version of the procedure [481]. It may be necessary to use two UCA injections, one to plan the procedure and a second to perform it. Alternatively, infusion may be used throughout the procedure. Besides the established use of CEUS in relation to percutaneous ablation and the potential benefit from intracavity CEUS, potential indications relate to accurate delineation of tissue vascularization.

\section{Avoiding biopsy of necrotic tissue}

By directing the biopsy needle towards contrast-enhanced areas within the target lesion, sampling from necrotic parts of the tumor can be avoided, resulting in an up to $15 \%$ increase in diagnostic accuracy reported in large tumors and liver metastases [482, 483] and in lung, neck and musculoskeletal tumors [484-488]. 


\section{Biopsy of poorly visualized or "invisible" lesions}

When a biopsy is required based on findings from CT, MR or PET$\mathrm{CT}$ imaging and the lesion is not clearly visualized or not visualized with B-mode US, CEUS may be helpful in two different ways: the target lesion suspected from previous imaging may become conspicuous on CEUS or additional lesions that are more accessible for biopsy may be visualized and biopsied [154, 489, 490].

\section{Further benefits from CEUS in interventional US}

CEUS may be used to:

a) Diagnose and monitor all stages of bleeding related to interventional procedures and guide percutaneous local application of hemostatic drugs [491, 492].

b) Improve breast cancer staging by identifying and guiding biopsy of the sentinel node after intradermal CEUS if axillary B-mode US is normal [413].

c) Improve visualization of poorly depicted fluid collections [153, 493].

d) Avoid biopsy of lesions if CEUS study unequivocally shows benign lesion, e. g. hepatic hemangioma [3].

\section{RECOMMENDATION 68}

CEUS can be helpful in avoiding necrotic tissue or identifying perfused tissue in the biopsy of tumors (LoE 2b, GoR C). Strong consensus (19/0/0, $100 \%)$

\section{RECOMMENDATION 69}

CEUS can be helpful in identifying biopsy targets inconspicuous on US (LoE 2b, GoR C). Strong Consensus (17/0/2, $100 \%$ )

\section{Interstitial Ablation Therapy}

\section{Background}

Interstitial ablation treatments are nonsurgical options for the management of confined tumors in the liver, kidney, prostate and uterus. CT and MR imaging represent the standard imaging modalities to assess therapeutic efficacy, but with evidence of the useful role of CEUS in the detection, guidance and confirmation of treatment success $[494,495]$.

\section{Kidney}

Thermal ablation is an accepted treatment option for unresectable renal cell carcinoma (RCC). The American Urological Association guidelines recommend ablation in patients with T1a disease $(<4 \mathrm{~cm})$ with high surgical risk, or in case of solitary kidney [496]. Until recently, both preprocedural diagnostic workup and postprocedural follow-up of patients referred for RCC ablation have included CT and/or MR imaging, whereas conventional B-mode US is frequently used for guidance during the ablation procedure.
CEUS is an important tool in the management of these patients and plays a decisive role in all stages of percutaneous ablation therapies [494, 495, 497 - 500].

\section{Study procedure}

\section{Pretreatment evaluation}

Diffuse heterogeneous enhancement in the arterial phase, washout in the late phase and perilesional rim-like enhancement are typical features of renal malignancies [499-501]. Identification of a pseudo-capsule predicts improved ablation efficacy [502], and inclusion of CEUS in the preprocedural imaging workup is useful to compare pre-ablation and post-ablation tumor viability.

\section{Intraprocedural evaluation}

Intraprocedural ablation evaluation is important but CEUS can be affected by gas artifacts of the ablation technique that can mask evaluation of tumor necrosis. Normally a 10- to 15-minute postablation period should be allowed before assessing the outcome [500]. CEUS has demonstrated high sensitivity, specificity and accuracy for the early detection of residual un-ablated tumor, comparable to CT and MR imaging [499 - 503].

\section{Follow-up}

Surveillance is recommended in patients who have undergone ablation due to a high local recurrence rate for tumors $>3 \mathrm{~cm}$ [494, 497 - 500], with suggested imaging surveillance every 6 months as CEUS can detect early recurrence not visible on B-mode US [496]. For the evaluation of residual or recurrent RCC, the sensitivity and specificity of CEUS are $82.2-100 \%$ and $96.6-100 \%$, respectively $[499,503,504]$. The concordance of CEUS with CT or MR imaging is between $80 \%$ and $100 \%$ [498, 499, 503, 505, 506].

\section{Prostate}

Interstitial ablation through high-intensity focused ultrasound (HIFU) can be applied in localized prostate cancer in patients at high surgical risk or for local recurrence after radiotherapy [507]. There is good concordance between MR and CEUS imaging, with CEUS being able to clearly and correctly identify the devascularized area of necrosis and the residual viable tissue, permitting immediate repeat treatment [508].

\section{Uterus}

CEUS in the intraprocedural evaluation of treatment response of benign uterine fibroids to US-guided HIFU demonstrated that CEUS correlated well with MR imaging. Four studies for the evaluation of the therapeutic efficacy of uterine fibroids compared the performance of CEUS to MR [509-511] or unenhanced US [512] with CEUS performing well compared to MR and better than B-mode US. 


\section{RECOMMENDATION 70}

CEUS may be used in the management of patients treated with ablation therapies including renal cell carcinoma (LoE 1b, GoR B), uterine fibroids (GOR C) and prostate cancer (GOR C). Strong consensus (18/1/0, $95 \%)$

\section{Miscellaneous}

\section{Intracavitary uses}

\section{Background}

Extravascular or intracavitary administration of UCAs may be used as a problem-solving tool, recognized in a number of reports as an adjunct to US-guided interventional techniques [493], with practical advice detailing the available concepts and techniques [209, 513].

\section{Study procedure}

No standard UCA dosage has been established for intracavitary applications. The range reported is $0.1 \mathrm{~mL}-1 \mathrm{~mL}$ SonoVue ${ }^{\mathrm{TM}}$ (or a few drops) diluted in $\geq 10 \mathrm{~mL} 0.9 \%$ normal saline. A higher UCA dose may be needed for high-frequency US transducers.

Injection into physiological cavities

\section{Imaging of tubal patency}

Originally performed using agitated saline infused into the uterine cavity, hystero-salpingo-sonography has a $12 \%$ false-negative patency rate [514]. Contrast-enhanced hystero-salpingo-contrast sonography (CE-HyCoSy) with SonoVue ${ }^{\mathrm{TM}}$ provides better specificity [514-516], but is low for the diagnosis of an occlusion [514]. CE-HyCoSy should only be performed if conventional hystero-salpingo-contrast sonography does not show patency.

\section{Detection of peritoneal-pleural communication}

The detection of direct connections between the abdominal and pleural cavities, hepatic hydrothorax, can be established in cirrhotic patients by injecting a UCA into the peritoneal cavity, early $(<2$ days) after thoracentesis and demonstrating UCA passage into the pleural cavity [517-519].

\section{CEUS-guided percutaneous transhepatic cholangiography}

CEUS-guided percutaneous cholangiography is able to delineate the biliary tree via an indwelling T-tube in place of the conventional fluoroscopic techniques, with the advantage of $3 \mathrm{D}$ techniques [210, $520-526]$. This technique allows for deployment at the point of care. UCA for endoscopic retrograde cholangiography (CEUS-ERC) has been reported [527, 528].

\section{Intracavitary CEUS for guiding percutaneous nephrostomy}

Intracavitary CEUS can guide percutaneous nephrostomy and assess complications and is able to confirm the needle or catheter position, evaluate the site of obstruction and assess catheterrelated complications. Patients with contraindications to iodinated contrast agents are suitable for this technique or at the point of care [529, 530].

\section{Salivary glands}

CEUS injected into the main duct of a salivary gland may be a diagnostic method to categorize obstructive diseases of the salivary glands. The salivary gland is cannulated with appropriate dilatators and the plastic tube of a peripheral vein catheter is inserted $[531,532]$.

Injection into non-physiological cavities

\section{CEUS for the imaging of fistula}

CEUS detection and classification of fistulas, irrespective of the underlying disease, is effective [533, 534]. The following conditions have been reported: rectovaginal fistulas via a transvaginal approach [208], vesico-intestinal fistulas via a transabdominal approach [535] and anal fistulas via the transrectal approach [533].

\section{CEUS for the imaging of abscesses}

Image-guided treatment of abscesses includes drainage with a needle or catheter, plus lavage [493]. Direct injection of a UCA through the needle or catheter has been reported to facilitate confirmation of correct needle or catheter position and allows evaluation of any communication between cavities in complex abscesses [493, 513, 536, 537].

\section{RECOMMENDATION 71}

Intracavitary CEUS allows identification of needle or catheter position, delineation of any cavity or duct, improved tracking of a fistula, optionally supplemented by intravenous CEUS (LoE 3, GoR C). Strong consensus (19/0/0, $100 \%$ )

\section{Free Tissue Transplants}

\section{Background}

Free flap reconstruction of complex defects after trauma, tumor resection, burns, or poor wound healing is able to restore the integrity of the defect and provide return of function. Despite technical refinement, flap loss due to vascular compromise occurs and is a serious complication. Early identification of vascular compromise and prompt revision permits early flap salvage, with CEUS being an ideal technique for the early detection of reduced vascularization [538-542]. CEUS is the only imaging method for the evaluation of dynamic changes of microvascularization during surgery and postoperative follow-up

\section{Study procedure}

High-frequency transducers ( $\geq 6 \mathrm{MHz}$ ) are used to evaluate the microcirculation of the cutaneous, subcutaneous, and deeper layers 
of free flaps most frequently using $1.2-2.4 \mathrm{~mL}$ SonoVue ${ }^{\mathrm{TM}}$. Postoperative TIC analysis allows calculation of peak and time to peak of enhancement and regional blood volume.

\section{Image Interpretation}

\section{Preoperative planning}

The blood vessels in the transplanted free flap are small (1 $2 \mathrm{~mm}$ ). The surgeon needs to know the integrity of the flow, precise number, course and position of these blood vessels in order to estimate the proportion with a good blood supply. Evaluation to determine time to peak (TTP), relative blood flow (rBF) and relative blood volume ( $\mathrm{rBV}$ ) as well as the evaluation of the critical microvascularization in the different layers of the free flaps is undertaken.

\section{Intraoperative imaging}

CEUS enables the identification of perforator vessels intraoperatively, detecting abnormalities, to allow a more accurate decision as to whether the entire flap is perfused and if the estimated flap size is correct.

\section{Postoperative monitoring}

The feeding vessels or, if there is a connection to a bypass, the anastomosis, as well as the free flap vessels is examined to identify thrombosis, embolism, twisting, kinking, or compression to confirm successful surgical salvage [541, 543].

\section{Critical microvascularization}

A significant difference between normally vascularized and compromised flaps can be observed most usefully with TTP and RBF [544]. For CEUS and CE-MRI, the mean signal increase of the TIC was significantly higher in ROls of normally perfused flaps compared to compromised flaps [545-549]. With CEUS, the exact size of the necrotic regions, hematoma or seroma can be evaluated by analyzing avascular areas.

\section{Limitations}

A limitation for the evaluation of flap perfusion is the time allowed after a bolus injection of the UCA. Continuous infusion may improve this, but this has not been evaluated.

\section{RECOMMENDATION 72}

CEUS may be used for the pre-, peri-, and postoperative evaluation of vascularization in free flap transplantation (LoE 2, GoR B). Strong consensus (19/0/0, $100 \%)$

\section{Lung}

\section{Background}

US of parenchymal lung lesions targets only those lesions abutting the pleura, and in this context, an accurate diagnosis is possible [550 -553]. CEUS in the evaluation of lung lesions is less well investigated. The lung parenchyma has a dual arterial system, the pulmonary arteries and the bronchial arteries. The ratio between blood supply from pulmonary arteries and bronchial arteries varies depending on the etiology of the underlying disease [554].

\section{Study Procedure}

The administered dose varies (SonoVue ${ }^{\mathrm{TM}} 2.4$, rarely $4.8 \mathrm{~mL}$ ) followed by a bolus of normal saline [554 - 557], with the enhancement continuously observed for at least 30 seconds. A time to enhancement of $<10$ seconds is indicative of a predominant supply from pulmonary arteries [555-557].

\section{Clinical applications}

CEUS might be a valuable tool to differentiate benign from malignant lesions $[555,557]$ and in the assessment of pulmonary embolic consolidation [555]. Studies are limited, with few patients to allow for determination of the diagnostic value of CEUS in the evaluation of lung lesions.

\section{Pneumonia}

Pneumonia is mainly supplied by the pulmonary artery, resulting in early (<10 seconds) homogenous enhancement [554, 557]. CEUS has also been reported to be a valuable tool for detecting and guiding drainage of abscess formation within pneumonia [558].

\section{Pulmonary embolism}

Embolic consolidations in patients with pulmonary embolism are reported to show absent or non-homogenous enhancement on CEUS, a consequence of variable degrees of bronchial arterial supply [555]. This feature could be helpful for distinguishing pulmonary infarction from pneumonia or compression atelectasis.

\section{Atelectasis}

Compression atelectasis is mainly supplied by the pulmonary artery and demonstrates early, marked enhancement on CEUS [556], whereas obstructive atelectasis presents with a delayed enhancement pattern [559]. Responsive vasoconstriction in obstructive atelectasis is considered responsible for the differences in enhancement pattern, but this is debatable [559].

\section{Lung cancer}

The enhancement pattern of lung cancer is variable [556], but there are suggestions that delayed (> 7.5 seconds) enhancement in neoplastic lesions might be a useful characterization of malignant pulmonary lesions [554]. CEUS may be used to avoid areas of necrosis in US-guided biopsies to increase diagnostic accuracy. 


\section{RECOMMENDATION 73}

CEUS may be used to delineate lung abscesses (LoE 3b, GoR C) and to guide US biopsy of non-necrotic areas of visualized lung lesions (LoE 3b, GoR C). Strong Consensus (16/0/3, $100 \%$ )

\section{Tumor Response Assessment}

\section{Background}

The advent of novel therapies targeting tumor angiogenesis and vascularity has highlighted the need for accurate and reproducible quantitative techniques to assess early changes in tumor vascularity [560]. However, as these therapies are predominantly cytostatic, current response assessment, which is based on interval evaluation of the tumor size using the Response Evaluation Criteria In Solid Tumors (RECIST) [561] is inadequate as it reflects only late changes and is unable to identify non-responders at an early time-point [562].

\section{Study procedure}

Dynamic contrast-enhanced US (DCE-US) can be performed using two different approaches with different results [563]:

\section{Bolus injection of a UCA with TIC analysis}

Single plane imaging is usually performed at $10-20$ frames per second for the duration of the enhancement. The average intensity within a region of interest (ROI) can be displayed as a function of time, i. e., a TIC which describes the wash-in and washout of the UCA in the ROI [564]. In addition, a second ROI can be placed in a reference tissue for comparison purposes [565]. The majority of clinical studies to date are based on this method.

Intravenous infusion of a UCA with disruption-replenishment analysis

The UCA is administered over 5 to 20 minutes. UCA is first imaged without being disrupted at a low $\mathrm{Ml}$, then the $\mathrm{Ml}$ is increased for a few frames, causing microbubble disruption. Immediately after that, the MI is returned to the non-disrupting level to observe the replenishment of the microbubbles into the ROI. Various models describe the echo-signal dynamics during the UCA-replenishment phase, which can be used for flow analysis [566]. Initially, monitoring for tumor response with UCAs relied on qualitative analyses [567], but new methodologies have been developed to produce more robust and semi-quantitative indices. Analyses of the TIC, including wash-in and washout times, can be performed with curve fitting to determine functional indices [568]. The main indices include: peak intensity ( $\mathrm{PI}$ ); area under the curve (AUC); area under the wash-in (AUWI); area under the washout (AUWO; all corresponding to blood volume); time to peak intensity (TPI); slope of the wash-in (SWI; both corresponding to blood flow); and mean transit time (MTT). No permeability information can be obtained because of the pure blood pool nature of microbubbles.

\section{Clinical application}

Early clinical trials employed qualitative analysis in the assessment of the response of different tumors such as gastrointestinal stromal tumor (GIST) or renal cell carcinoma [569 - 572]. More recently, there have been studies using semi-quantitative techniques with UCA bolus injection in renal cell carcinoma, hepatocellular carcinoma (HCC) and GIST [573-575]. Studies showed that two indices representing blood volume correlated with the RECIST response; one study on renal cell carcinoma demonstrated a correlation of such indices with Progression Free Survival and Overall Survival [573]. The results could not be reproduced in a study testing the disruption-replenishment technique versus Progression Free Survival assessed by the RECIST method [566].

A multicenter study of various types of tumors treated with anti-angiogenic therapies, such as metastatic renal cell carcinoma, GIST, colon cancer, melanoma, breast cancer and HCC, with approximately half of the tumors being located outside the liver, is currently being conducted in 539 patients with more than 2000 DCE-US scans. A quality score was proposed in a standardized acquisition [576], with AUC being the best parameter. A decrease of $40 \%$ at one month was significantly correlated with Freedom From Progression (FFP) and also with Overall Survival which is the best end-point for the validation of a biomarker [577]. There is now emerging evidence that DCE-US may be used with appropriate tools to differentiate between responders and non-responders at an earlier stage than conventional methods and this potentially allows tailoring of the treatment regimen, particularly changing treatment for non-responders. DCE-US has been endorsed by the European Medical Oncology Society to assess response under biological therapy for GIST [578].

\section{RECOMMENDATION 74}

Dynamic CEUS can be utilized to assess response to biologic therapy in metastatic GIST and in other tumors (LoE 1b, GoR A). Strong Consensus (15/0/4, 100\%)

\section{Conflict of Interest}

Fabrizio Calliada: Congress participation support, Toshiba, Guerbet, Bayer, Hitachi, Mindray

Vito Cantisani: Congress participation support, Toshiba, Guerbet, Bayer, Hitachi, Mindray

Dirk Clevert: Speaker honoraria for Siemens, Philips, Samsung Falk, Bracco; Advisory Board Member Siemens, Philips, Samsung, Bracco; Research grants, Siemens, Philips, Samsung, Bracco

David Cosgrove: Speaker honoraria, Bracco, Toshiba, SuperSonic, BK Medical, Carestream; Advisory Board Member, Toshiba, Carestream; Research grant, Carestream; Congress participation support, Bracco, Toshiba

Annamarie Dagenello: Speaker honoraria, Bracco

Christoph F Dietrich: Speaker honoraria, Bracco, Hitachi, GE, Mindray, Supersonic, Pentax, Olympus, Fuji, Covidien, Boston Scientific, AbbVie, MSD, Falk Foundation, Novartis, Roche; Advisory Board Member, Hitachi and Mindray; Research grant, Bracco, Hitachi, GE, Siemens, Mindray, SuperSonic; Congress participation support, IPSEN 
Odd Helge Gilja: Speaker honoraria, GE Healthcare Takeda AS and Meda AS

Christian Jenssen: Speaker honoraria, Bracco, Hitachi, Toshiba, Falk

Foundation, Covidien; Research grant, Novartis

Nathalie Lassau: Speaker honoraria, Bracco, Toshiba; Congress participation support, Bracco, Toshiba

Edward Leen: Research equipment support, Philips Healthcare \& SuperSonic Imaging

Maria Franca Meloni: Speaker honoraria, Bracco

Christian Pállson Nolsøe: Speaker honoraria and congress participation support, GE Healthcare and Neovitalis

Mirko D’Onofrio: Speaker honoraria, Bracco, Siemens; Advisor Board

Member, Bracco, Siemens, Congress participation support, Bracco

Fabio Piscaglia: Speaker honoraria Bracco, Bayer; Advisory Board Mem-

ber, Bayer; Research support, Esaote

Maija Radzina: Speaker honoaria, Bayer, Covidien; Congress participation support, Bayer

Adrian Saftiou: Speaker honoraria, Pentax Medical Singapore Ltd; Consulting/Advisory board, Mediglobe Corporation Gmbh; Congress participation support, Hitachi Medical Systems UK

Paul Sidhu: Speaker honoraria, Siemens, Bracco, Hitachi, Philips and GE Hans-Peter Weskott: Speaker honoraria for Bracco, Samsung and GE The following members declared no conflicts of interest: Eva Bartels, Michele Bertolotto, Francesca Drudi, Simon Freeman, Christopher Harvey, Ernst Michael Jung, Andrea Klauser, Carlos Nicolau, Francesco Prada, Luca Savelli, Hessel Wijkstra

\section{Acknowledgements}

The authors thank the EFSUMB's Lynne Rudd for her continuing support of the guidelines.

We also thank the following companies for funding a consensus meeting of the authors held in London in July 2016, at which we agreed on recommendations: bk/Ultrasound, Echosens, Esaote SpA, GE Healthcare, Hitachi Medical Systems, Philips Healthcare, Shenzhen Mindray Bio-medical Electronics Co., Ltd, Siemens Heathineers, Supersonic, Toshiba Medical and Bracco SpA.

Representatives of these companies were in attendance at this meeting to assist with technical product information but did not take part in the writing of this manuscript or the recommendations.

\section{References}

[1] Albrecht T, Blomley MJK, Bolondi L et al. Guidelines for the use of contrast agents in ultrasound. Ultraschall in Med 2004; 25: 249-256

[2] Claudon M, Cosgrove D, Albrecht T et al. Guidelines and Good Clinical Practice Recommendations for Contrast Enhanced Ultrasound (CEUS) Update 2008. Ultraschall in Medizin 2008; 29: 28-44

[3] Claudon M, Dietrich CF, Choi BI et al. Guidelines and good clinical practice recommendations for contrast enhanced ultrasound (CEUS) in the liver - update 2012. Ultraschall in Med 2013; 34: 11-29

[4] Piscaglia F, Nolsoe C, Dietrich CF et al. The EFSUMB guidelines and recommendations on the clinical practice of contrast enhanced ultrasound (CEUS): update 2011 on non-hepatic applications. Ultraschall in Med 2012; 32: 33 - 59

[5] Sidhu PS, Cantisani V, Deganello A et al. Role of contrast-enhanced ultrasound (CEUS) in paediatric practice: An EFSUMB position statement. Ultraschall in Med 2017; 38: 33-43

[6] Sidhu PS, Choi BI, Bachmann Nielsen M. The EFSUMB guidelines and recommendations on the clinical practice of contrast enhanced ultrasound (CEUS): a new dawn for the escalating use of this ubiquitous technique. Ultraschall in Med 2012; 32: 5 -7
[7] Food \& Drug Administration. MARCH 2016 - APPROVED DRUG PRODUCT LIST. 2016 http://www.fda.gov/downloads/drugs/developmentapprovalprocess/ucm071120.pdf

[8] Bracco. Lumason Prescribing Information. 2016 http://imaging.bracco. com/sites/braccoimaging.com/files/technica_sheet_pdf/US-20160330LUMASON-PI_0.pdf

[9] Seitz K, Strobel D. A Milestone: Approval of CEUS for Diagnostic Liver Imaging in Adults and Children in the USA. Ultraschall in Med 2016; 37 : $229-232$

[10] Education and Practical Standards Committee E. Minimal Training recommendations for the practice of medical ultrasound. Ultraschall in Med 2006; 27: 79-95

[11] Education and Practical Standards Committee E. Minimum Training Requirements for the Practice of Medical Ultrasound in Europe. Appendix 14: (CEUS) Contrast Enhanced Ultrasound. Ultraschall in Med 2010; 31: $426-427$

[12] Quaia E, Alaimo V, Baratella E et al. Effect of Observer Experience in the Differentiation Between Benign and Malignant Liver Tumors After Ultrasound Contrast Agent Injection. J Ultrasound Med 2010; 29: 25-36

[13] Greis C. Technical aspects of contrast-enhanced ultrasound (CEUS) examinations: tips and tricks. Clin Hemorheol Microcirc 2014; 58: 89-95

[14] Blomley MJK, Sidhu PS, Cosgrove DO et al. Do different types of liver lesions differ in their uptake of the microbubble contrast agent SH U 508A in the late liver phase? Early experience. Radiology 2001; 220: 661-667

[15] Bryant TH, Blomley MJK, Albrecht T et al. Liver phase uptake of a liver specific microbubble improves characterization of liver lesions: a prospective multi-center study. Radiology 2004; 232: 392 - 399

[16] Edey AJ, Ryan SM, Beese RC et al. Ultrasound imaging of liver metastases in the delayed parenchymal phase following administration of Sonazoid $^{\mathrm{TM}}$ using a destructive mode technique (Agent Detection Imaging ${ }^{\mathrm{TM}}$ ). Clin Radiol 2008; 63: 1112 - 1120

[17] Piscaglia F, Bolondi L. The safety of SonoVue in abdominal applications: retrospective analysis of 23188 investigations. Ultrasound Med Biol 2006; 32: $1369-1375$

[18] Main ML. Ultrasound contrast agent safety: from anecdote to evidence. JACC Cardiovasc Imaging 2009; 2: 1057 - 1059

[19] Main ML, Goldman JH, Grayburn PA. Thinking Outside the box:The Ultrasound Contrast Controversy. J Am Coll Cardiol 2007; 50: 2434-2437

[20] Tang C, Fang K, Guo Y et al. Safety of Sulfur Hexafluoride Microbubbles in Sonography of Abdominal and Superficial Organs: Retrospective Analysis of 30222 Cases. J Ultrasound Med 2017; 36: 531 - 538

[21] Wilson SR, Burns PN. Microbubble-enhanced US in body imaging: What role? Radiology 2010; 257: 24-39

[22] Kitzman DW, Goldman ME, Gilliam LD et al. Efficacy and safety of the novel ultrasound contrast agent perflutren (Definity) in patients with suboptimal baseline left ventricular echocardiographic images. Am J Cardiol 2000; 86: 669-674

[23] Cochran ST, Bomyea K, Sayre JW. Trends in adverse events after IV administration of contrast media. Am J Roentgenol 2001; 176: 1385- 1388

[24] Hunt CH, Hartman RP, Hesley GK. Frequency and Severity of Adverse Effects of lodinated and Gadolinium Contrast Materials: Retrospective Review of 456930 Doses. Am J Roentgenol 2009; 193: 1124-1127

[25] Committee for Medicinal Products for Human Use (CHMP). Assessment report EMA/84084/2014. SonoVue. international non-properietary name: sulfur hexafluoride, Procedure No. EMEA/H/C/000303/II/0025. 2014

[26] Darge K, Papadopoulu F, Ntoulia A et al. Safety of contrast-enhanced ultrasound in children for non-cardiac applications: a review by the Society for Pediatric Radiology (SPR) and the International Contrast Ultrasound Society (ICUS). Pediatr Radiol 2013; 43: 1063-1073 
[27] Harvey C], Alsafi A, Kuzmich S et al. Role of US contrast agents in the assessment of indeterminate solid and cystic lesions in native and transplant kidneys. Radiographics 2015; 35: 1419-1430

[28] Barr RG, Peterson C, Hindi A. Evaluation of indeterminate renal massess with contrast-enhanced US: a diagnostic performance study. Radiology 2014; 271: $133-142$

[29] Harkanyi Z. Potential applications of contrast-enhanced ultrasound in pediatric patients. Ultrasound Clin North Am 2013; 8: 403-422

[30] Sellars ME, Deganello A, Sidhu PS. Paediatric contrast-enhanced ultrasound (CEUS); a technique that requires co-operation for rapid implementation into clinical practice. Ultraschall in Med 2014; 35: 203 - 206

[31] Caruso G, Salvaggio G, Campisi A et al. Bladder Tumor Staging: Comparison of Contrast-Enhanced and Gray-Scale Ultrasound. Am J Roentgenol 2010; 194: 151 - 156

[32] Drudi FM, Di Leo N, Maghella F et al. CEUS in the study of bladder, method, administration and evaluation, a technical note. J Ultrasound 2014; 17: $57-63$

[33] Drudi FM, Cantisani V, Liberatore M et al. Role of Low-Mechanical Index CEUS in the Differentiation between Low and High Grade Bladder Carcinoma: a Pilot Study. Ultraschall in Med 2010; 31: 589- 595

[34] Wang XH, Wang Y], Lei CG. Evaluating the perfusion of occupying lesions of kidney and bladder with contrast-enhanced ultrasound. Clin Imaging 2011; 35: $447-451$

[35] Nicolau C, Bunesch L, Sebastia C et al. Diagnosis of bladder cancer: contrast-enhanced ultrasound. Abdom Imaging 2010; 35: 494- 503

[36] Nicolau C, Bunesch L, Peri L et al. Accuracy of contrast-enhanced ultrasound in the detection of bladder cancer. Br J Radiol 2011; 84: 1091 1099

[37] Correas JM, Claudon M, Tranquart F et al. The kidney: imaging with microbubble contrast agents. Ultrasound Q 2006; 22: 53 -66

[38] Tsuruoka K, Yasuda T, Koitabashi K et al. Evaluation of Renal Microcirculation by Contrast-Enhanced Ultrasound With Sonazoid ${ }^{\mathrm{TM}}$ as a Contrast Agent Comparison Between Normal Subjects and Patients With Chronic Kidney Disease. Int Heart ] 2010; 51: 176-182

[39] Ma F, Cang Y, Zhao B et al. Contrast-enhanced ultrasound with SonoVue could accurately assess the renal microvascular perfusion in diabetic kidney damage. Nephrol Dial Transplant 2012; 27: 2891 - 2898

[40] Bertolotto M, Martegani A, Aiani L et al. Value of contrast-enhanced ultrasonography for detecting renal infarcts proven by contrast enhanced CT. A feasibility study. Eur Radiol 2008; 18: 376-383

[41] Yusuf GT, Sellars ME, Huang DY et al. Cortical Necrosis Secondary to Trauma in a Child: Contrast-enhanced ultrasound comparable to Magnetic Resonance Imaging. Pediatr Radiol 2014; 44: 484-487

[42] Mazziotti S, Zimbaro F, Pandolfo A et al. Usefulness of contrast-enhanced ultrasonography in the diagnosis of renal pseudotumors. Abdom Imaging 2010; 35: 241 - 245

[43] Quaia E, Bertolotto M, Cioffi V et al. Comparison of contrast-enhanced sonography with unenhanced sonography and contrast-enhanced CT in the diagnosis of malignancy in complex renal masses. Am J Roentgenol 2008; 191: 1239 - 1249

[44] Clevert DA, Minaifar N, Weckbach S et al. Multislice computed tomography versus contrast-enhanced ultrasound in evaluation of complex cystic renal masses using the Bosniak classification system. Clin Hemorheol Microcirc 2008; 39: 171 - 178

[45] Park BK, Kim B, Kim SH et al. Assessment of cystic renal masses based on Bosniak classification: comparison of CT and contrast-enhanced US. Eur ] Radiol 2007; 61: 310-314

[46] Ascenti G, Mazziotti S, Zimbaro G et al. Complex Cystic Renal Masses: Characterization with Contrast-enhanced US. Radiology 2007; 243: $158-165$
[47] Bertolotto M, Cicero C, Perrone R et al. Renal masses with equivocal enhamcement at CT: characterization with contrast-enhanced ultrasound. Am J Roentgenol 2015; 205: W557 - W565

[48] Fontanilla T, Minaya J, Cortes $C$ et al. Acute complicated pyelonephritis: contrast-enhanced ultrasound. Abdominal Imaging 2012; 37: 639-646

[49] Ignee A, Straub B, Brix D et al. The value of contrast enhanced ultrasound (CEUS) in the characterisation of patients with renal masses. Clin $\mathrm{He}-$ morheol Microcirc 2010; 46: 275-290

[50] Papadopoulou F, Ntoulia A, Siomou E et al. Contrast-enhanced voiding urosonography with intravesical administration of a second-generation ultrasound contrast agent for diagnosis of vesicoureteral reflux: prospective evaluation of contrast safety in 1010 children. Pediatr Radiol 2014; 44: $719-728$

[51] Darge K. Voiding urosonography with US contrast agents for the diagnosis of vesicureteric reflux in children. I. Procedure. Pediatr Radiol 2008; 38: $40-53$

[52] Darge K. Voiding urosonography with US contrast agents for the diagnosis of vesicureteric reflux in children. II. Comparison with radiological examinations. Pediatr Radiol 2008; 38: 54-63

[53] Ascenti G, Zimbaro G, Mazziotti S et al. Harmonic US imaging of vesicoureteric reflux in children: usefulness of a second generation US contrast agent. Pediatr Radiol 2004; 34: 481 - 487

[54] Wong LS, Tse KS, Fan TW et al. Voiding urosonography with secondgeneration ultrasound contrast versus micturating cystourethrography in the diagnosis of vesicoureteric reflux. Eur J Pediatr 2014; 173: 1095 1101

[55] Duran C, Riego J, Riera L et al. Voiding urosonography including urethrosonography: high-quality examinations with an optimised procedure using a second-generation US contrast agent. Pediatr Radiol 2012; 42: $660-667$

[56] Kis E, Nyitrai A, Varkonyi I et al. Voiding urosonography with secondgeneration contrast agent versus voiding cystourethrography. Pediatr Nephrol 2010; 25: 2289-2293

[57] Papadopoulou F, Anthopoulou A, Siomou E et al. Harmonic voiding urosonography with a second-generation contrast agent for the diagnosis of vesicoureteral reflux. Pediatr Radiol 2009; 39: 239-244

[58] Darge K. Voiding urosonography with US contrast agent for the diagnosis of vesicoureteric reflux in children: an update. Pediatr Radiol 2010; 40: $956-962$

[59] Giordano M, Marzolla R, Puteo F et al. Voiding urosonography as first step in the diagnosis of vesicoureteral reflux in children: a clinical experience. Pediatr Radiol 2007; 37: 674-677

[60] Duran C, Valera A, Alguersuari A et al. Voiding urosonography: the study of the urethra is no longer a limitation of the technique. Pediatr Radiol 2009; 39: 124-131

[61] Lebowitz RL, Olbing H, Parkkulainen KV et al. International system of radiographic grading of vesicourteric reflux. Internationa Reflux Study in Children. Pediatr Radiol 1985; 15: 105-109

[62] Fernaíndez-Ibieta M, Parrondo-Muiros C, Fernaíndez-Masaguer LC et al. Urosonografija miccional con contraste de segunda generaci+' n como herramienta primaria en el estudio de la vija urinaria superior e inferior en pediatrija. Estudio Piloto. Actas Urolgicas Espaolas 2016; 40: 183 189

[63] Wozniak MM, Wieczorek AP, Pawelec A et al. Two-dimensional (2D), three-dimensional static (3D) and real-time (4D) contrast enhanced voiding urosonography (ceVUS) versus voiding cystourethrography (VCUG) in children with vesicoureteral reflux. Eur J Radiol 2016; 85: $1238-1245$

[64] Duran C, Riego J, Riere L. Serial voiding urosonography: a safe technique for the study of the entire urinary tract in children. Radiologia 2013; 55 : $160-166$

[65] Kmetec A, Bren AF, Kandus A et al. Contrast-enhanced ultrasound voiding cystography as a screening examination for vesicoureteral reflux in 
the follow-up of renal transplant recipient: a new approach. Nephrol Dial Transplant 2001; 16: 120-123

[66] Valentini AL, De Gaetano AM, Minordi LM et al. Contrast-enhanced Voiding US for Grading of Reflux in Adult Patients Prior to Antireflux Ureteral Implantation. Radiology 2004; 233: 35 - 39

[67] Kenda RB, Kenig A, Novijan G et al. Cyclic voiding urosonography for detecting vesicoureteric reflux in renal transplant recipients. Nephrol Dial Transplant 2001; 16: 2229-2231

[68] Jaffer OS, Sidhu PS. Contrast-enhanced ultrasonography of the testes. Ultrasound Clin North Am 2013; 8: 509 - 523

[69] Albrecht T, Lotzof K, Hussain HK et al. Power Doppler US of the normal prepubertal testis: does it live up to its promises? Radiology 1997; 203: $227-231$

[70] Moschouris H, Stamatiou K, Lampropoulou E et al. Imaging of the acute scrotum; is there a place for contrast-enhanced ultrasonography? Int Braz J Urol 2009; 35: $702-705$

[71] Yusuf T, Sellars ME, Kooiman GG et al. Global testicular infarction in the presence of epididymitis. Clinical features, appearances on grayscale, color Doppler, and contrat-enhanced sonography, and histologic correlation. J Ultrasound Med 2013; 32: 175-180

[72] Bilagi P, Sriprasad S, Clarke JL et al. Clinical and ultrasound features of segmental testicular infarction: Six-year experience from a single centre. Eur Radiol 2007; 17: $2810-2818$

[73] Fernandez-Perez GC, Tardaguila FM, Velasco M et al. Radiologic Findings of Segmental Testicular Infarction. Am J Roentgenol 2005; 184: 1587 1593

[74] Sriprasad SI, Kooiman GG, Muir GH et al. Acute sgmental testicular infarction: differentiation from tumour using high frequency colour Doppler ultrasound. Br J Radiol 2001; 74: 965 - 967

[75] Bertolotto M, Derchi LE, Sidhu PS et al. Acute segmental testicular infarction at contrast-enhanced ultrasound: early features and changes during follow-up. Am J Roentgenol 2011; 196: 834-841

[76] Lung PF, Jaffer OS, Sellars ME et al. Contrast enhanced ultrasound (CEUS) in the evaluation of focal testicular complications secondary to epidiymitis. Am J Roentgenol 2012; 199: W345-W354

[77] Patel K, Huang DY, Sidhu PS. Metachronous Bilateral Segmental Testicular Infarction: Multi-parametric Ultrasound imaging with Grey-scale Ultrasound, Doppler Ultrasound, Contrast Enhanced Ultrasound (CEUS) and Real-time Tissue Elastography (RTE). J Ultrasound 2014; 17: 233 238

[78] Bhatt S, Dogra VS. Role of US in testicular and scrotal trauma. Radiographics 2008; 28: 1617 - 1629

[79] Valentino M, Bertolotto M, Derchi L et al. Role of contrast enhanced ultrasound in acute scrotal diseases. Eur Radiol 2011; 21: 1831 - 1840

[80] Lobianco R, Regine R, De Siero M et al. Contrast-enhanced sonography in blunt scrotal trauma. J Ultrasound 2011; 14: 188-195

[81] Hedayati V, Sellars ME, Sharma DM et al. Contrast-enhanced ultrasound in testicular trauma: role in directing exploration, debridement and organ salvage. $\mathrm{Br}$ J Radiol 2012; 85: e65-e68

[82] Yusuf GT, Konstantatou E, Sellars ME et al. Multiparametric sonography of testicular hematomas. Features on grayscale, color Doppler, and contrast-enhanced sonography and strain elastography. J Ultrasound Med 2015; 34: 1319-1328

[83] Rafailidis V, Robbie H, Konstantatou E et al. Sonographic imaging of extra-testicular focal lesions: comparison of grey-scale, colour Doppler and contrast-enhanced ultrasound. Ultrasound 2016; 24: 23 - 33

[84] Horstman WG, Melson GL, Middleton WD et al. Testicular tumours: Findings with color Doppler US. Radiology 1992; 185: 733 - 737

[85] Bhatt S, Rubens DJ, Dogra VS. Sonography of benign intrascrotal lesions. Ultrasound Q 2006; 22: 121 - 136

[86] Winter TC. There is a mass in the scrotum - what does it mean? Evaluation of the scrotal mass. Ultrasound Q 2009; 25: 195-205
[87] Patel K, Sellars ME, Clarke JL et al. Features of testicular epidermoid cysts on contrast enhanced ultrasound and real time elastography. J Ultrasound Med 2012; 31: 1115-1122

[88] Huang DY, Sidhu PS. Focal testicular lesions: colour Doppler ultrasound, contrast-enhanced ultrasound and tissue elastography as adjuvants to the diagnosis. $\mathrm{Br}$ J Radiol 2012; 85: S41 - S53

[89] Lock G, Schmidt C, Helmich F et al. Early experience with contrast enhanced ultrasound in the diagnosis of testicular masses; a feasibility study. Urology 2011; 77: 1049-1053

[90] Isidori AM, Pozza C, Gianfrilli D et al. Differential diagnosis of nonpalpable testicular lesions: qualitative and quantitave contrast-enhanced US of benign and malignant testicular tumors. Radiology 2014; 273: 606 618

[91] Drudi FM, Maghella F, Martino G et al. Detection of small testicular masses in monorchid patients using US, CPDUS, CEUS and US-guided biopsy. J Ultrasound 2016; 19: 25-28

[92] Drudi FM, Valentino M, Bertolotto $M$ et al. CEUS time intensity curves in the differentiation between Leydig cell carcinoma nd seminoma: a multicenter study. Ultraschall in Med 2016; 37: 201 - 205

[93] Lock G, Schoder C, Schmidt C et al. Contrast-enhanced ultrasound and real time elastography for the diagnosis of benign leydig cell tumors of the testis - a single centre report on 13 cases. Ultraschall in Med 2014; 35: $534-539$

[94] Gaur S, Bhatt S, Derchi L et al. Spontaneous intratesticular hemorrhage. Two case descriptions and a brief review of the literature. J Ultrasound Med 2011; 30: $101-104$

[95] Bertolotto M, Cantisani V, Valentino $M$ et al. Pitfalls in Imaging for Acute Scrotal Pathology. Semin in Roentgenol 2016; 51: 60-69

[96] Russo G, Mischi M, Scheepens W et al. Angiogenesis in prostate cancer: onset, progression and imaging. BJU International 2012; 110: E794E808

[97] Mitterberger MJ, Aigner F, Horninger W et al. Comparative efficiency of contrast-enhanced colour Doppler ultrasound targeted versus systematic biopsy for prostate cancer detection. Eur Radiol 2010; 20: 2791 2796

[98] Aigner F, Pallwein L, Mitterberger M et al. Contrast-enhanced ultrasonography using cadence-contrast pulse sequencing technology for targeted biopsy of the prostate. BJU International 2009; 103: 458-463

[99] Sano F, Terao H, Kawahara T et al. Contrast-enhanced ultrasonography of the prostate: various imaging findings that indicate prostate cancer. BJU International 2011; 107: 1404-1410

[100] Strazdina A, Krumina G, Sperga M. The value and limitations of contrast-enhanced ultrasound in detection of prostate cancer. Anticancer Res 2011; 31: $1421-1426$

[101] Sano F, Uemura H. The Utility and Limitations of Contrast-Enhanced Ultrasound for the Diagnosis and Treatment of Prostate Cancer. Sensors (Basel) 2015; 15: 4947 -4957

[102] Smeenge M, Barentsz J, Cosgrove D et al. Role of transrectal ultrasonography (TRUS) in focal therapy of prostate cancer: report from a Consensus Panel. BJU International 2012; 110: 942 - 948

[103] Schalk SG, Demi L, Smeenge M et al. 4-D spatiotemporal analysis of ultrasound contrast agent dispersion for prostate cancer localization: a feasibility study. IEEE Trans UltrasonFerroelectri Freq Control 2015; 62: $839-851$

[104] Postema AW, Frinking PJA, Smeenge M et al. Dynamic contrast-enhanced ultrasound parametric imaging for the detection of prostate cancer. BJU International 2016; 117: 598-603

[105] Postema A, Mischi M, de la Rosette ] et al. Multiparametric ultrasound in the detection of prostate cancer: a systematic review. World J Urol 2015; 33: 1651 - 1659 
[106] Harvey CI, Sidhu PS, Bachmann-Nielsen M. Contrast-enhanced ultrasound in renal transplants: applications and future directions. Ultraschall in Med 2013; 34: 319-321

[107] McArthur C, Baxter GM. Current and potential renal applications of contrast-enhanced ultrasound. Clin Radiol 2012; 67: 909-922

[108] Girometti R, Stocca E, Granata A et al. Impact of contrast-enhanced ultrasound in patients with renal function impairment. World J Radio 2017; 28: $10-16$

[109] Granata A, Andrulli S, Fiorini F et al. Diagnosis of acute pyelonephritis by contrast-enhanced ultrasonography in kidney transplant patients. Nephrol Dial Transplant 2011; 26: 715-720

[110] Stenberg B, Talbot D, Khurram M et al. A new technique for assessing renal transplant perfusion preoperatively using contrast-enhanced ultrasound (CEUS) and three-dimensional ultrasound (3DUS) - a porcine modelpilot study. Ultraschall in Med 2011; 32: E8-E13

[111] Stenberg B, Chandler C, Wyrley-Birch H et al. Post-Operative 3-Dimensional Contrast-Enhanced Ultrasound (CEUS) Versus Tc99m-DTPA in the Detection of Post-Surgical Perfusion Defects in Kidney Transplants: Preliminary Findings. Ultraschall in Med 2014; 35: 273 -278

[112] Fernandez CP, Ripolles T, Martinez MJ et al. Diagnosis of acute cortical necrosis in renal transplantation by contrast-enhanced ultrasound: a preliminary experience. Ultraschall in Med 2013; 34: 338-342

[113] Kay DH, Mazonakis M, Geddes C et al. Ultrasonic microbubble contrast agents and the transplant kidney. Clin Radiol 2009; 64: 1081 - 1087

[114] Jin Y, Yang C, Wu S et al. A novel simple onoinvasive index to predict renal transplant acute rejection by contrast-enhanced ultrasonography. Transplantation 2015; 99: 636-641

[115] Tranquart F, Mercier L, Frinking $P$ et al. Perfusion quantification in contrast-enhanced ultrasound (CEUS) ready for research projects and routine clinical use. Ultraschall in Med 2012; 33: S31 - S38

[116] Wang X, Yu Z, Guo R et al. Assessment of postoperative perfusion with contrast-enhanced ultrasonography in kidney transplantation. Int J Clin Exp Med 2015; 8: 18399-18405

[117] Dietrich CF, Wehrmann T, Hoffmann C et al. Detection of the Adrenal Glands by Endoscopic or Transabdominal Ultrasound. Endoscopy 1997; 29: 859-864

[118] Trojan J, Schwarz W, Sarrazin C et al. Role of Ultrasonography in the Detection of Small Adrenal Masses. Ultraschall in Med 2002; 23: 96 100

[119] Friedrich-Rust M, Schneider G, Bohle RM et al. Contrast-enhanced sonography of adrenal masses; differentiation of adenomas and nonadenomatous lesions. Am J Roentgenol 2008; 191: 1852-1860

[120] Friedrich-Rust M, Glasemann T, Polta A et al. Differentiation between benign and malignant adrenal mass using contrast-enhanced ultrasound. Ultraschall in Medizin 2011; 32: 460-471

[121] Dietrich CF, Ignee A, Barreiros AP et al. Contrast-enhanced ultrasound for imaging of adrenal masses. Ultraschall in Med 2010; 31: 163-168

[122] Slapa RZ, Kasperlik-Zaluska AA, Migda B et al. Application of parametric ultrasound contrast agent perfusion studies for differentiation of hyperplastic adrenal nodules from adenomas - Initial study. Eur ] Radiol 2015; 84: 1432 - 1435

[123] Al Bunni F, Deganello A, Sellars ME et al. Contrast-enhanced ultrasound (CEUS) appearances of an adrenal phaeochromocytoma in a child with Von Hippel-Lindau disease. J Ultrasound 2014; 17: 307 - 311

[124] Schreiber-Dietrich D, Buchhorn R, Dietrich CF. Blickdiagnose Nebennierentumor (Phächromozytom). Pädiat Prax 2014; 83: 239-248

[125] Denbow ML, Welsh AW, Taylor M] et al. Twin fetuses: intravascular microbubble US contrast agent administration - early experience. Radiology 2000; 214: $724-728$

[126] Denbow ML, Blomley MJK, Cosgrove DO et al. Ultrasound microbubble contrast angiography in monochorionic twin fetuses. Lancet 1997; 349: 773
[127] Song Y, Yang J, Shen K. Preoperative evaluation of endometrial carcinoma by contrast-enhanced ultrasonography. BJOG 2008; 116: 294 298

[128] Testa AC, Ferrandina G, Fruscella E et al. The Use of Contrasted Transvaginal Sonography in the Diagnosis of Gynecologic Diseases: A Preliminary Study. J Ultrasound Med 2005; 24: 1267 - 1278

[129] Lieng M, Qvigstad E, Dahl GF et al. Flow differences between endometrial polyps and cancer: a prospective study using intravenous contrast-enhanced transvaginal color flow Doppler and three-dimensional power Doppler ultrasound. Ultrasound Obstet Gynecol 2008; 32: 935- 940

[130] Dorenberg EJ, Hol PK, Jakobsen J et al. Improved infarction rates in fibroids after the introduction of contrast-enhanced ultrasound during uterine artery embolization. Acta Radiologica 2012; 53: 34 - 38

[131] Sconfienza LM, Lacelli F, Gandolfo N et al. Contrast-enhanced ultrasound (CEUS) assessment of superselective uterine fibroid embolization (SUFE): Preliminary experience. J Ultrasound 2008; 11: 158-161

[132] Orden MR, Jurvelin JS, Kirkinen PP. Kinetics of a US Contrast Agent in Benign and Malignant Adnexal Tumors. Radiology 2003; 226: 405 410

[133] Marret H, Sauget S, Giraudeau B et al. Contrast-Enhanced Sonography Helps in Discrimination of Benign From Malignant Adnexal Masses. J Ultrasound Med 2004; 23: 1629-1639

[134] Testa AC, Timmerman D, Exacoustos C et al. The role of CnTI-SonoVue in the diagnosis of ovarian masses with papillary projections: a preliminary study. Ultrasound Obstet Gynecol 2007; 29: 512 - 516

[135] Testa AC, Timmerman D, Van Belle $V$ et al. Intravenous contrast ultrasound examination using contrast-tuned imaging ( $\mathrm{CnTI})$ and the contrast medium SonoVue fro discrimination between benign and malignant adnexal masses with solid components. Ultrasound Obstet Gynecol 2009; 34: 699-710

[136] D’Onofrio M, Biagioli E, Gerardi C et al. Diagnostic performance of contrast-enhanced ultrasound (CEUS) and contrast-enhanced endoscopic ultrasound (ECEUS) for the differentation of pancreatic lesions: a systematic review and meta-analysis. Ultraschall in Med 2014; 35 : $515-521$

[137] D'Onofrio M, Canestrini S, De Robertis R et al. CEUS of the pancreas: Still research or the standard of care. Eur J Radiol 2015; 84: 1644 1649

[138] Dietrich CF, Braden B, Hocke M et al. Improved characterisation of solitary solid pancreatic tumours using contrast enhanced transabdominal ultrasound. J Cancer Res Clin Oncol 2008; 134: 635-643

[139] D’Onofrio M, Megibow AJ, Faccioli N et al. Comparison of Contrast-Enhanced Sonography and MRI in Displaying Anatomic Features of Cystic Pancreatic Masses. Am J Roentgenol 2007; 189: 1435-1442

[140] Fan Z, Li Y, Yan K et al. Application of contrast-enhanced ultrasound in the diagnosis of solid pancreatic lesionsGÇöA comparison of conventional ultrasound and contrast-enhanced CT. Eur J Radiol 2013; 82: $1385-1390$

[141] Kitano M, Kudo M, Maekawa K et al. Dynamic imaging of pancreatic diseases by contrast enhanced coded phase inversion harmonic ultrasonography. Gut 2004; 53: $854-859$

[142] D'Onofrio M, Malago R, Zamboni G et al. Contrast-enhanced ultrasonography better identifies pancreatic tumor vascularization than helical CT. Pancreatology 2005; 5: 398-402

[143] Faccioli N, D’Onofrio M, Malaga R et al. Resectable Pancreatic Adenocarcinoma: Depiction of Tumoral Margins at Contrast-Enhanced Ultrasonography. Pancreas 2008; 37: 265-268

[144] Grossjohann HS, Rappeport ED, Jensen C et al. Usefulness of contrastenhanced transabdominal ultrasound for tumor classification and tumor staging in the pancreatic head. Scand j Gastroenterol 2010; 45: 917-924 
[145] D’Onofrio M, Zamboni G, Tognolini A et al. Mass-forming pancreatitis: Value of contrast-enhanced ultrasonography. World J Gastroenterol 2006; 12: $4181-4184$

[146] Kersting S, Konopke R, Kersting F et al. Quantitative Perfusion Analysis of Transabdominal Contrast-Enhanced Ultrasonography of Pancreatic Masses and Carcinomas. Gastroenterol 2009; 137: 1903-1911

[147] Numata K, Ozawa Y, Kobayashi N et al. Contrast-enhanced sonography of pancreatic carcinoma; correlations with pathological findings. J Gastroenterol 2005; 40: 631-640

[148] D’Onofrio M, Barbi E, Dietrich CF et al. Pancreatic multicenter ultrasound study (PAMUS). Eur J Radiol 2012; 81: 630-638

[149] Serra C, Felicani C, Mazzotta E et al. Contrast-Enhanced Ultrasound in the Differential Diagnosis of Exocrine Versus Neuroendocrine Pancreatic Tumors. Pancreas 2013; 42: 871-877

[150] Wang Y, Yan K, Fan Z et al. Contrast-Enhanced Ultrasonography of Pancreatic Carcinoma: Correlation with Pathologic Findings. Ultrasound Med Biol 2016; 42: 891 - 898

[151] D’Onofrio M, Crosara S, Signorini M et al. Comparison between CT and CEUS in the Diagnosis of Pancreatic Adenocarcinoma. Ultraschall in Med 2013; 34: $377-381$

[152] Bernatik T, Seitz K, Blang W et al. Unclear focal liver lesions in contrastenhanced ultrasonography - lessons to be learned from the DEGUM multicenter study for the characterization of liver tumors. Ultraschall in Med 2010; 31: 587 - 596

[153] Sidhu PS, Brabrand K, Cantisani V et al. EFSUMB Guidelines on Interventional Ultrasound (INVUS) Part II Diagnostic Ultrasound-Guided Interventional Procedures (Short Version). Ultraschall in Med 2016; 36 : $566-580$

[154] Wei Y, Yu XL, Liang P et al. Guiding and Controlling Percutaneous Pancreas Biopsies with Contrast-Enhanced Ultrasound: Target Lesions Are Not Localized on B-Mode Ultrasound. Ultrasound Med Biol 2015; 41 : $1561-1569$

[155] Tawada K, Yamaguchi T, Kobayashi A et al. Changes in Tumor Vascularity Depicted by Contrast-Enhanced Ultrasonography as a Predictor of Chemotherapeutic Effect in Patients With Unresectable Pancreatic Cancer. Pancreas 2009; 38: 30 -35

[156] Kobayashi A, Yamaguchi T, Ishihara T et al. Evaluation of vascular signal in pancreatic ductal carcinoma using contrast enhanced ultrasonography: effect of systemic chemotherapy. Gut 2005; 54: 1047

[157] D’Onofrio M, Mansueto G, Falconi M et al. Neuroendocrine pancreatic tumor. Abdom Imaging 2004; 29: 246-258

[158] Malago R, D’Onofrio M, Zamboni GA et al. Contrast-Enhanced Sonography of Nonfunctioning Pancreatic Neuroendocrine Tumors. Am J Roentgenol 2009; 192: 424-430

[159] Falconi M, Bartsch DF, Eriksson B et al. ENETS consesus guidelines for the management of patients with digestive neuroendocrine neoplasms of the digestive system: well-differentiated pancreatic nonfunctioning tumors. Neuroendocrinology 2012; 95: 120-134

[160] Fan Z, Yan K, Wang Y et al. Application of Contrast-Enhanced Ultrasound in Cystic Pancreatic Lesions Using a Simplified Classification Diagnostic Criterion. Biomed Res Int 2015; 2015: 974621

[161] Vasile TA, Socaciu M, Stanluga R et al. Added value of intravenous contrast-enhanced ultrasoundfor characterization of cystic pancreatic masses: a prospective study on 37 patients. Med Ultrason 2012; 14: $108-114$

[162] Rickes S, Wermke W. Differentiation of cystic pancreatic neoplasms and pseudocysts by conventional and echo-enhanced ultrasound. J Gastroenterol Hepatol 2004; 19: 761 - 766

[163] Itoh T, Hirooka Y, Itoh A et al. Usefulness of Contrast-Enhanced Transabdominal Ultrasonography in the Diagnosis of Intraductal Papillary Mucinous Tumors of the Pancreas. Am J Gastroenterol 2005; 100: $144-152$
[164] Pezzilli R, Serra C, Calculli L et al. Three-Dimensional Contrast-Enhanced Ultrasonography of Intraductal Papillary Mucinous Neoplasms of the Pancreas: A Comparison With Magnetic Resonance Imaging. Pancreas 2013; 42: 1164-1168

[165] Buscarini E, Pezzilli R, Cannizzaro R et al. Italian consensus guidelines for the diagnostic work-up and follow-up of cystic pancreatic neoplasms. Dig Liver Dis 2014; 46: 479-493

[166] D’Onofrio M, Gallotti A, Pozzi Mucelli R. Imaging techniques in pancreatic tumors. Expert Rev Med Devices 2010; 7: 257-273

[167] Ripolles T, Martinez MJ, Lopez E et al. Contrast-enhanced ultrasound in the staging of acute pancreatitis. Eur Radiol 2010; 20: 2518-2523

[168] Ardelean M, Sirli R, Sporea I et al. Contrast enhanced ultrasound in the pathology of the pancreas. A monocentric experience. Med Ultrason 2014; 16: $325-331$

[169] Vitali F, Pfeifer L, Janson C et al. Quantitative perfusion analysis in pancreatic contrast enhanced ultrasound (DCE-US): a promising tool for the differentiation between autoimmune pancreatitis and pancreatic cancer. Z Gastroenterol 2015; 53: 1175-1181

[170] Mauch M, Blank W, Kunze G et al. Importance of Abdominal Ultrasound in 17 Patients with Histologically Confirmed Autoimmune Pancreatitis (AIP). Ultraschall in Med 2015; 36: 248- 254

[171] Ito T, Kenmochi T, Nishikawa T et al. A Novel Screening Test for Detecting Graft Thrombosis After Pancreatic Transplantation Using Contrast-enhanced Ultrasonography With Sonazoid. Transplant Proc 2014 46: $1917-1919$

[172] Rennert J, Farkas S, Georgieva M et al. Identification of early complications following pancreas and renal transplantation using contrast enhanced ultrasound (CEUS) - first results. Clin Hemorheol Microcirc 2014; $58: 343-352$

[173] Aboutaleb E, Leen E, Hakim N. Assessment of Viability of the Pancreas for Transplantation Using Contrast-Enhanced Ultrasound. Transplant Proc 2011; 43: 418-421

[174] Kersting S, Ludwig S, Ehehalt F et al. Contrast-Enhanced Ultrasonography in Pancreas Transplantation. Transplantation 2013; 95: 209-214

[175] Nylund K, hausken T, Gilja $\mathrm{OH}$. Ultrasound and inflammatory bowel disease. Ultrasound Q 2010; 26: 3-15

[176] Gorce JM, Arditi D, Schneider M. Influence of bubble size distribution on the echogenicity of ultrasound contrast agents: a study of SonoVue. Invest Radiol 2000; 35: 661-671

[177] Plikat K, Klebi F, Buchner C et al. Evaluation of intestinal hyperaemia in inflammed bowel by high resolution contarst harmonic imaging (CHI). Ultraschall in Med 2000; 25: 257-262

[178] Girlich C, Jung EM, lesalnieks I et al. Quantitative assessment of bowel wall vascularisation in Crohn's disease with contrast-enhanced ultrasound and perfusion analysis. Clin Hemorheol Microcirc 2009; 43: $141-148$

[179] Kratzer W, Schmidt SA, Mittrach C et al. Contrast-enhanced wideband harmonic imaging ultrasound (SonoVue): a new technique for quantifying bowel wall vascularity in Crohn's disease. Scand j Gastroenterol 2005; 40: $985-991$

[180] De Pascale A, Garofalo G, Perna M et al. Contrast-enhanced ultrasonography in Crohn's disease. Radiol Med 2006; 111: 539- 550

[181] De Franco A, MArzo M, Felice C et al. Ileal Crohn's disease: CEUS determination of activity. Abdom Imaging 2012; 37: 359-368

[182] Bialecki M, Bialecka A, Laskowska K et al. Contrast-enhanced ultrasonography in Crohn's disease diagnostic. Hepatogastroenterol 2015; 62: $927-932$

[183] Roccarina D, Garcovich M, Ainora ME et al. Diagnosis of bowel diseases: the role of imaging and ultrasonography. World J Gastroenterol 2013; 19: $2144-2153$ 
[184] Chiorean L, Schreiber-Dietrich D, Braden B et al. Ultrasonographic imaging of inflammatory bowel disease in pediatric patients. World ] Gastroenterol 2015; 21: 5231 - 5241

[185] Schreyer AG, Finkenzeller T, Gossmann H et al. Microcirculation and perfusion with contrast enhanced ultrasound (CEUS) in Crohn's disease: first results with linear contrast harmonic imaging ( $\mathrm{CHI})$. Clin Hemorheol Microcirc 2008; 40: 143-155

[186] Pauls S, Gabelmann A, Schmidt SA et al. Evaluting bowel wall vascularity in Crohn's disease: a comparison of dynamic MRI and wideband harmonic imaging contrat-enhanced low MI ultrasound. Eur Radiol 2006; 16: $2410-2417$

[187] Malago R, D’Onofrio M, Mantovani W et al. Contrast-enhanced ultrasonography (CEUS) vs. MRI of the small bowel in the evaluation of Crohn's disease activity. La Radiologia Medica 2012; 117: 268 - 281

[188] Badea R, Socaciu M, Ciobanu L et al. Contrast-enhanced ultrasonography (CESU) for the evaluation of the inflammation of the digestive tract wall. J Gastrointestin Liver Dis 2010; 19: 439-444

[189] Robotti D, Cammarota T, Debani P et al. Activity of Crohn disease: value of Color-Power-Doppler and contrast-enhanced ultrasonography. Abdo Imaging 2004; 29: 648-652

[190] Serra C, Menozzi G, Labate AMM et al. Ultrasound assessment of vascularization of the thickened terminal ileum wall in Crohn's disease patients using a low-mechanical index real-time scanning technique with a second generation ultrasound contrast agent. Eur J Radiol 2007; 62: $114-121$

[191] Migaleddu V, Scanu AM, Quaia E et al. Contrast-Enhanced Ultrasonographic Evaluation of Inflammatory Activity in Crohn's Disease. Gastroenterology 2009; 137: 43-52

[192] Girlich C, Schacherer D, Jung EM et al. Comparison between quantitative assessment of bowel wall vascularization by contrast-enhanced ultrasound and results of histopathological scoring in ulcerative colitis. Int J Colorectal Dis 2012; 27: $193-198$

[193] Ripolles T, Martinez MJ, Paredes JM et al. Crohn Disease: Correlation of Findings at Contrast-enhanced US with Severity at Endoscopy. Radiology 2009; 253: $241-248$

[194] Girlich C, Jung EM, Huber E et al. Comparison between Preoperative Quantitative Assessment of Bowel Wall Vascularization by ContrastEnhanced Ultrasound and Operative Macroscopic Findings and Results of Histopathological Scoring in Crohn's Disease. Ultraschall in Med 2011; 32: $154-159$

[195] Romanini L, Passamonti M, Navarria M et al. Quantitative analysis of contrast-enhanced ultrasonography of the bowel wall can predict disease activity in inflammatory bowel disease. Eur J Radiol 2014; 83 $1317-1323$

[196] Saevik F, Nylund K, Hausken T et al. Bowel Perfusion Measured with Dynamic Contrast-enhanced Ultrasound Predicts Treatment Outcome in Patients with Crohn's Disease. Inflamm Bowel Dis 2014; 20: 2029 2037

[197] Guidi L, De Franco A, De Vitis I et al. Contrast-enhanced ultrasonography with SonoVue after infliximab therapy in Crohn's disease. Eur Rev Med Pharmacol Sci 2006; 10: 23 -26

[198] Quaia E, Migaleddu V, Baratella E et al. The diagnostic value of small bowel wall vascularity after sulfur hexafluoride-filled microbubble injection in patients with Crohn's disease. Correlation with the therapeutic effectiveness of specific anti-inflammatory treatment. Eur ] Radiol 2009; 69: 438-444

[199] Paredes JM, Ripolles T, Cortis X et al. Contrast-enhanced ultrasonography: Usefulness in the assessment of postoperative recurrence of Crohn's disease. J Crohns Colitis 2013; 7: 192-201

[200] Serafin Z, Bialécki M, Bialécka A et al. Contrast-enhanced Ultrasound for Detection of Crohn's Disease Activity: Systematic Review and Metaanalysis. J Crohns Colitis 2016; 10: $354-362$
[201] Ma X, Li Y, Jia H et al. Contrast-Enhanced Ultrasound in the Diagnosis of Patients Suspected of Having Active Crohn's Disease: Meta-analysis. Ultrasound Med Biol 2015; 41: 659-668

[202] Kratzer W, von Tirpitz C, Mason R et al. Contrast-Enhanced Power Doppler Sonography of the Intestinal Wall in the Differentiation of Hypervascularized and Hypovascularized Intestinal Obstructions in Patients With Crohn's Disease. J Ultrasound Med 2002; 21: 149-157

[203] Schirin-Sokhan R, Winograd R, Tischendorf S et al. Assessment of Inflammatory and Fibrotic Stenoses in Patients with Crohns Disease Using Contrast-Enhanced Ultrasound and Computerized Algorithm: A Pilot Study. Digestion 2011; 83: 263-268

[204] Nylund K, Jirik R, Mezl M et al. Quantitative Contrast-Enhanced Ultrasound Comparison Between Inflammatory and Fibrotic Lesions in Patients with Crohn's Disease. Ultrasound Med Biol 2013; 39: 1197 1206

[205] Kinkel H, Michels G, Jaspers N. Ultraschall zur Diagnostik und Verlaufskontrolle chronisch-entzundlicher Darmerkrankungen. Dtsch Med Wochenschr 2015; 140: 46-50

[206] Schlottmann K, Kratzer W, Scholmerich J. Doppler ultrasound and intravenous contrast agents in gastrointestinal tract disorders: current role and future implications. Eur J Gastroenterol Hepatol 2005; 17 : $263-275$

[207] Ripolles T, Martinez-Perez MJ, Paredes JM et al. Contrast-enhanced ultrasound in the differentiation between phlegmon and abscess in Crohn's disease and other abdominal conditions. Eur J Radiol 2013; 82: e525-e531

[208] Henrich W, Meckies J, Friedmann W. Demonstration of a recto-vaginal fistula with ultrasound contrast medium Ehovist. Ultrasound Obstet Gynecol 2000; 15: 148-149

[209] Heinzmann A, Muller T, Leitlein J et al. Endocavity contrast enhanced ultrasound (CEUS) - work in progress. Ultraschall in Med 2012; 33: $76-84$

[210] Ignee A, Cui X, Schuessler G et al. Percutaneous transhepatic cholangiography and drainage using extravascular contrast enhanced ultrasound. Z Gastroenterol 2015; 53: 385-390

[211] Nonnenmacher S, Muller T, Haug U et al. Nachweis einer iliacoenteralen Fistel als seltene Ursache einer unteren gastrointestinalen Blutung mittels Kontrastmittelsonografie (CEUS). Ultraschall in Med 2013; 34: 478-480

[212] Gao JM, Tang SS, Fu W et al. Signet-ring cell carcinoma of ampulla of Vater: Contrast-enhanced ultrasound findings. World J Gastroenterol 2009; 15: 888-891

[213] Wang Y, Li L, Wang YX et al. Time-Intensity Curve Parameters in Recta Cancer Measured Using Endorectal Ultrasonography with Sterile Coupling Gels Filling the Rectum: Correlations with Tumor Angiogenesis and Clinicopathological Features. Biomed Res Int 2014; 2014: 587806

[214] Stock K, Hann von Weyhern C, Slotta-Huspenina J et al. Microcirculation of subepithelial gastric tumors using contras-enhanced ultrasoun. Clin Hemorheol Microcirc 2010; 45: 225 - 232

[215] Dorffel Y, Wermke W. Neuroendocrine Tumors: Characterization with Contrast-Enhanced Ultrasonography. Ultraschall in Med 2008; 29 : $506-514$

[216] Cao BS, Li L, Li YX et al. Assessment of abnormal bowel perfusion using contrast-enhanced ultrasonography after small bowel transplantation: A case report. J Clin Ultrasound 2013; 41: 370-272

[217] Benedetti E, Bruno B, McDonald GB et al. Prospective qualitative and quantitative non-invasive evaluation of intestinal acute GVHD by contrast-enhanced ultrasound sonography. Bone Marrow Transplant 2013; 48: 1421 - 1428

[218] Schreyer AG, Landfried K, Zorger $\mathrm{N}$ et al. Transmural penetration of intravenously applied microbubbles during contrast-enhanced ultrasound as a new diagnostic feature in patients with GVHD of the bowel. Bone Marrow Transplant 2011; 46: 1006-1011 
[219] Bachmann C, Klibanov AL, Olson TS et al. Targeting Mucosal Addressin Cellular Adhesion Molecule (MAdCAM)-1 to Noninvasively Image Experimental Crohns Disease. Gastroenterology 2006; 130: 8-16

[220] Zink F, Kratzer W, Schmidt S et al. Comparison of Two High-End Ultrasound Systems for Contrast-Enhanced Ultrasound Quantification of Mural Microvascularity in Crohn's Disease. Ultraschall in Med 2016; 37 : $74-81$

[221] Neesse A, Huth J, Kunsch S et al. Contrast-enhanced ultrasound pattern of splenic metastases. A retrospective study in 32 patients. Ultraschall in Medizin 2010; 31: $264-269$

[222] Catalano O, Lobianco R, Sandomenico F et al. Real-time contrast enhanced ultrasound of the spleen: examination technique and preliminary clinical experience. Radiol Med 2003; 106: 338-356

[223] Lim AKP, Patel N, Eckersley RJ et al. Evidence for Spleen-specific Uptake of a Microbubble Contrast Agent: A Quantitative Study in Healthy Volunteers. Radiology 2004; 231: 785 - 788

[224] Catalano O, Cusati B, Nunziata A et al. Real-time, contrast-specific sonography imaging of acute splenic disorders: a pictorial review. Emerg Radiol 2004; 11: 15-21

[225] Catalano O, Sandomenico F, Vallone G et al. Contrast-enhanced sonography of the spleen. Semin Ultrasound CT MR 2006; 27: 426-433

[226] Omar A, Freeman S. Contrast-enhanced ultrasound of teh spleen. Ultrasound 2016; 24: 41 - 49

[227] Catalano O, Sandomenico F, Matarazzo I et al. Contrast-Enhanced Sonography of the Spleen. Am J Roentgenol 2005; 184: 1150-1156

[228] Peddu P, Shah M, Sidhu PS. Splenic abnormalities: a comparative review of ultrasound, microbubble enhanced ultrasound and computed tomography. Clin Radiol 2004; 59: 777-792

[229] Stang A, Keles H, Hentschke S et al. Differentiation of Benign From Malignant Focal Splenic Lesions Using Sulfur Hexafluoride-Filled Microbubble Contrast-Enhanced Pulse-Inversion Sonography. Am J Roentgenol 2009; 193: 709-721

[230] Gorg C. The forgotten organ: Contrast enhanced sonography of the spleen. Eur J Radiol 2007; 64: 189-201

[231] Gorg C, Graef C, Bert T. Contrast-enhanced sonography for differential diagnosis of an inhomogenous spleen of unknown cause in patients with pain in the left upper quadrant. J Ultrasound Med 2006; 25: 729 734

[232] Gorg C, Bert T. Second-Generation Sonographic Contrast Agent for Differential Diagnosis of Perisplenic Lesions. Am J Roentgenol 2006; 186: $621-626$

[233] Kim SH, Lee JM, Lee JY et al. Contrast-Enhanced Sonography of Intrapancreatic Accessory Spleen in Six Patients. Am J Roentgenol 2007; 188: $422-428$

[234] Bertolotto M, Quaia E, Zappetti R et al. Differential diagnosis between splenic nodules and peritoneal metastases with contrast-enhanced ultrasound based on signal-intensity characteristics during the late phase. Radiol Med 2009; 114: 42 - 51

[235] Li W, Liu G, Wang W et al. Real-time contrast enhanced ultrasound imaging of focal splenic lesions. Eur J Radiol 2014; 83: 646-653

[236] von Herbay A, Barreiros AP, Ignee A et al. Contrast-enhanced ultrasonography with SonoVue. Differentiation between benign and malignant lesions of the spleen. J Ultrasound Med 2009; 28: 421 -434

[237] Cai DM, Parajuly SS, Ling WW et al. Diagnostic value of contrast enhanced ultrasound for splenic artery complications following acute pancreatitis. World J Gastroenterol 2014; 20: 1088-1094

[238] Menozzi G, Maccabruni V, Gabbi E et al. Contrast-enhanced Ultrasound Evaluation of Splenic Embolization in Patients with Definite Left-Sided Infective Endocarditis. Ultrasound Med Biol 2013; 39: 2205-2210

[239] Ignee A, Cui X, Hirche T et al. Percutaneous biopsies of splenic lesions a clinical and contrast based algorithm. Clin Hemorheol Microcirc 2014; 58: 529- 541
[240] Chen LW, Chien RN, Yen CL et al. Splenic tumour: a clinicopathological study. Int J Clin Pract 2004; 58: $924-927$

[241] Yu X, Yu J, Liang P et al. Real-time contrast-enhanced ultrasound in diagnosing of focal spleen lesions. Eur J Radiol 2012; 81: 430-436

[242] Stang A, Keles H, Hentschke S et al. Incidentally Detected Splenic Lesions in Ultrasound: Does Contrast-Enhanced Ultrasonography Improve the Differentiation of Benign Hemangioma/Hamartoma from Malignant Lesions? Ultraschall in Med 2011; 32: 582 - 592

[243] Taibbi A, Bartolotta TV, Matranga D et al. Splenic Hemangiomas: Contrast-Enhanced Sonographic Findings. J Ultrasound Med 2012; 31: $543-553$

[244] Picardi M, Soricelli A, Pane F et al. Contrast-enhanced Harmonic Compound US of the Spleen to Increase Staging Accuracy in Patients with Hodgkin Lymphoma: A Prospective Study. Radiology 2009; 251: 574 582

[245] Tafuto S, Catalano O, Barba G et al. Real-time contrast-enhanced specific ultrasound in staging and follow-up of splenic lymphomas. Fron Biosci 2006; 11: $2224-2229$

[246] ten Kate GL, van den Oord SCH, Sijbrands EJG et al. Current status and future developments of contrast-enhanced ultrasound of carotid atherosclerosis. J Vasc Surg 2013; 57: 539-546

[247] Ventura CAP, da Silva ES, Cerri GG et al. Can contrast-enhanced ultrasound with second-generation contrast agents replace computed tomography angiography for distinguishing between occlusion and pseudo-occlusion of the internal carotid artery? Clinics (Sao Paulo) 2015; 70: 1 -6

[248] Clevert DA, Sommer WH, Zengel P et al. Imaging of carotid arterial diseases with contrast-enhanced ultrasound (CEUS). Eur J Radiol 2011; 80: $68-76$

[249] Shalhoub ], Owen DRJ, Gauthier T et al. The use of contrast enhanced ultrasound in carotid arterial disease. Eur J Vasc Endovasc Surg 2010; 39: $381-387$

[250] Clevert DA, Sommer WH, Heick A et al. Doplex and contrast enhanced ultrasound (CEUS) in evaluation of in-stent restenosis after carotid stenting. Clin Hemorheol Microcirc 2011; 48: 199-208

[251] Li Z], Luo XH, Du LF. Identification of carotid artery dissection by contrast enhanced ultrasonography. A case report. Med Ultrason 2015; 17: $564-565$

[252] Clevert DA, Kubisch C, Meimarakis G et al. Improved Visualization of Carotid-Jugular Arteriovenous Fistula by Contrast-Enhanced Ultrasound. Ultraschall in Med 2010; 31: 610-612

[253] Jaff MR, Goldmakher GV, Lev MH et al. Imaging of the carotid arteries: the role of duplex ultrasonography, magnetic resonance arteriography, and computerized tomographic arteriography. Vasc Med 2008; 13: $281-292$

[254] van den Oord SCH, ten Kate GL, Sijbrands EJG et al. Effect of Carotid Plaque Screening Using Contrast-Enhanced Ultrasound on Cardiovascular Risk Stratification. Am J Cardiol 2013; 111: 754 - 759

[255] ten Kate GL, Sijbrands EJ, Staub D et al. Noninvasive Imaging of the Vulnerable Atherosclerotic Plaque. Curr Probl Cardiol 2010; 35: 556 591

[256] ten Kate GL, van Dijk AC, van den Oord SCH et al. Usefulness of Contrast-Enhanced Ultrasound for Detection of Carotid Plaque Ulceration in Patients With Symptomatic Carotid\&\#xa0;Atherosclerosis. Am J Cardiol 2013; 112: $292-298$

[257] Coli S, Magnoni M, Sangiorgi G et al. Contrast-Enhanced Ultrasound Imaging of Intraplaque Neovascularization in Carotid Arteries: Correlation With Histology and Plaque Echogenicity. J Am Coll Cardiol 2008; 52: $223-230$

[258] Giannoni MF, Vicenzini E, Citone M et al. Contrast Carotid Ultrasound for the Detection of Unstable Plaques with Neoangiogenesis: A Pilot Study. Eur J Vasc Endovasc Surg 2009; 37: 722 - 727 
[259] Zhang Q, Li C, Han H et al. Spatio-temporal Quantification of Carotid Plaque Neovascularization on Contrast Enhanced Ultrasound: Correlation with Visual Grading and Histopathology. Eur J Vasc Endovas Surg 2015; 50: 289-296

[260] Li C, He W, Guo D et al. Quantification of Carotid Plaque Neovascularization Using Contrast-Enhanced Ultrasound With Histopathologic Validation. Ultrasound Med Biol 2014; 40: 1827 - 1833

[261] Vavuranakis M, Sigala F, Vrachatis DA et al. Quantitative analysis of carotid plaque vasa vasorum by CEUS and correlation with histology after endarterectomy. VASA 2013; 42: 184-195

[262] Owen DR, Shalhoub J, Miller S et al. Inflammation within carotid atherosclerotic plaque: assessment with late-phase contrast-enhanced US. Radiology 2010; 255: 638-644

[263] Shalhoub J, Monaco C, Owen DRJ et al. Late-Phase Contrast-Enhanced Ultrasound Reflects Biological Features of Instability in Human Carotid Atherosclerosis. Stroke 2011; 42: 3634-3636

[264] Staub D, Schinkel AFL, Coll B et al. Contrast-Enhanced Ultrasound Imaging of the Vasa Vasorum: From Early Atherosclerosis to the Identification of Unstable Plaques. JACC: Cardiovasc Imaging 2010; 3: $761-771$

[265] Staub D, Patel MB, Tibrewala A et al. Vasa Vasorum and Plaque Neovascularization on Contrast-Enhanced Carotid Ultrasound Imaging Correlates With Cardiovascular Disease and Past Cardiovascular Events. Stroke 2010; 41: $41-47$

[266] Ritter MA, Theismann K, Schmiedel M et al. Vascularization of carotid plaque in recently symptomatic patients is associated with the occurrence of transcranial microembolic signals. Eur ] Neurol 2013; 20: $1218-1221$

[267] van den Oord SCH, Akkus Z, Renaud G et al. Assessment of carotid atherosclerosis, intraplaque neovascularization, and plaque ulceration using quantitative contrast-enhanced ultrasound in asymptomatic patients with diabetes mellitus. Eur Heart J Cardiovasc Imaging 2014; 15: $1213-1218$

[268] Kim HS, Woo JS, Kim BY et al. Biochemical and clinical correlation of intraplaque neovascularization using contrast-enhanced ultrasound of the carotid artery. Atherosclerosis 2014; 233: 579-583

[269] van den Oord SCH, Akkus Z, Roeters van Lennep JE et al. Assessment of subclinical atherosclerosis and intraplaque neovascularization using quantitative contrast-enhanced ultrasound in patients with familial hypercholesterolemia. Atherosclerosis 2013; 231: 107-113

[270] Nakamura J, Nakamura T, Deyama J et al. Assessment of carotid plaque neovascularization using quantitative analysis of contrast-enhanced ultrasound imaging is useful for risk stratification in patients with coronary artery disease. Int J Cardiol 2015; 195: 113-119

[271] Deyama J, Nakamura T, Takishima I et al. Contrast-Enhanced Ultrasound Imaging of Carotid Plaque Neovascularization Is Useful for Identifying High-Risk Patients With Coronary Artery Disease. Circ J 2013; 77: 1499-1507

[272] Schinkel AFL, van den Oord SCH, van der Steen AFW et al. Utility of contrast-enhanced ultrasound for the assessment of the carotid artery wall in patients with Takayasu or giant cell arteritis. Eur Heart ] Cardiovasc Imaging 2014; 15: 541 - 546

[273] Possemato N, Macchioni P, Germano G et al. Clinical images: PET-CT and contrast-enhanced ultrasound in TakayasuGÇÖs arteritis. Rheumatology(Oxford) 2014; 53: 447

[274] Perren F, Poglia D, Landis T et al. Vertebral artery hypoplasia: a predisposing factor for posterior circulation stroke? Neurology 2007; 68: $65-67$

[275] Caplan LR. Arterial occlusions: does size matter? J Neuro Neurosurg Psych 2007; 78: 916

[276] Catalano O, Lobianco R, Cusati B et al. Contrast-enhanced sonography for diagnosis of ruptured abdominal aortic aneurysm. Am J Roentgenol 2005; 184: $423-427$
[277] Clevert DA, Stickel M, Johnson T et al. Imaging of aortic abnormalities with contrast-enhanced ultrasound. A pictorial comparison with CT. Eur Radiol 2007; 17: 2991 - 3000

[278] Pennell RC, Hollier LH, Lie JT et al. Inflammatory abdominal aortic aneurysms: A thirty-year review. J Vasc Surg 1985; 2: 859-869

[279] Clevert DA, Schick K, Chen MH et al. Role of contrast enhanced ultrasound in detection of abdominal aortic abnormalities in comparison with multislice computed tomography. Clin Med J (Eng) 2009; 122: $858-864$

[280] Thurnher M, Cejna M. Imaging of aortic stent-grafts and endoleaks. Radiol Clin North Am 2002; 40: 799-833

[281] Clevert DA, Sommer WH, Meimerakis G et al. Contrast-enhanced ultrasound compared with multislice computed tomography for endovascular aneurysm repair surveillance. Ultrasound 2011; 19: 11 - 19

[282] Clevert DA, Horng A, Clevert DA et al. Contrast-enhanced ultrasound versus conventional ultrasound and MS-CT in the diagnosis of abdominal aortic dissection. Clin Hemorheol Microcirc 2009; 43: 129-139

[283] d'Audiffret A, Desgranges P, Kobeiter DH et al. Follow-up evaluation of endoluminally treated abdominal aortic aneurysms with duplex ultrasonography: Validation with computed tomography. J Vasc Surg 2001; 33: $42-50$

[284] Sommer WH, Hoffmann RT, Becker CR et al. Comparison of time-resolved CT angiography, contrast-enhanced ultrasound and digital subtraction angiography in a patient with a small type II endoleak after endovascular aneurysm repair. Clin Hemorheol Microcirc 2010; 45: $19-25$

[285] Jung EM, Rennert J, Fellner C et al. Detection and Characterization of Endoleaks Following Endovascular Treatment of Abdominal Aortic Aneurysms using Contrast Harmonic Imaging (CHI) with Quantitative Perfusion Analysis (TIC) Compared to CT Angiography (CTA). Ultraschall in Med 2010; 31: $564-570$

[286] Napoli V, Bargellini I, Sardella SG et al. Abdominal Aortic Aneurysm: Contrast-enhanced US for Missed Endoleaks after Endoluminal Repair. Radiology 2004; 233: 217 -225

[287] Clevert DA, Gurtler VM, Meimarakis G et al. Classification of endoleaks in the follow-up after EVAR using the time-to-peak of the contrast agent in CEUS examination. Clin Hemorheol Microcirc 2013; 55: 183 - 191

[288] Ten Bosch JA, Rouwet EV, Peters CTH et al. Contrast-enhanced Ultrasound versus Computed Tomographic Angiography for Surveillance of Endovascular Abdominal Aortic Aneurysm Repair. J Vasc Interv Radiol 2010; 21: 638-643

[289] Gurtler VM, Sommer WH, Meimarakis G et al. A comparison between contrast-enhanced ultrasound imaging and multislice computed tomography in detecting and classifying endoleaks in the follow-up after endovascular aneurysm repair. J Vasc Surg 2013; 58: 340-345

[290] Sidhu PS, Allan PL, Cattin F et al. Diagnostic efficacy of SonoVue(R), a second generation contrast agent, in the assessment of extracranial carotid or peripheral arteries using colour and spectral Doppler ultrasound: a multicentre study. $\mathrm{Br}$ J Radiol 2006; 79: 44 - 51

[291] Pfister K, Kasprzak PM, Apfelbeck H et al. Stellenwert der Kontrastmittelsonografie in der Gefabchirurgie. Zentralbl Chir 2014; 139: 518 524

[292] Rubenthaler J, Reiser M, Clevert DA. Diagnostic vascular ultrasonography with the help of color Doppler and contrast-enhanced ultrasonography. Ultrasonography 2016; 35: 289-301

[293] Seidel G, Meairs S. Ultrasound Contrast Agents in Ischemic Stroke. Cerebrovasc Dis 2009; 27: 25-39

[294] Seidel G, Kaps M. Harmonic imaging of the vertebrobasilar system. Stroke 1997; 28: 1610-1613

[295] Postert T, Braun B, Meves S et al. Contrast-enhanced transcranial colorcoded sonography in acute hemispheric brain infarction. Stroke 1999; 30: $1819-1826$ 
[296] Droste DW, Jurgens R, Weber S et al. Benefit of Echocontrast-Enhanced Transcranial Color-Coded Duplex Ultrasound in the Assessment of Intracranial Collateral Pathways. Stroke 2000; 31: 920-923

[297] Eyding J, Krogias C, Schollhammer M et al. Contrast-Enhanced Ultrasonic Parametric Perfusion Imaging Detects Dysfunctional Tissue at Risk in Acute MCA Stroke. J Cerebl Blood Flow Metab 2006; 26: 576 582

[298] Bartels E, Henning S, Wellmer A et al. Evaluation of Cerebral Perfusion Deficit in Stroke Patients Using New Transcranial Contrast Imaging CPS Technology - Preliminary Results. Ultraschall in Med 2005; 26: 478-486

[299] Bartels E, Bittermann H]. Transcranial Contrast Imaging of Cerebral Perfusion in Patients With Space-Occupying Intracranial Lesions. J Ultrasound Med 2006; 25: 499-507

[300] Seidel G, Canger H, Meyer-Wiethe K et al. On the Ability of Ultrasound Parametric Perfusion Imaging to Predict the Area of Infarction in Acutelschemic Stroke. Ultraschall in Med 2006; 27: 543 - 548

[301] Krogias C, Hennebohl C, Geier B et al. Transcranial Ultrasound Perfusion Imaging and Perfusion-MRI - A Pilot Study on the Evaluation of Cerebral Perfusion in Severe Carotid Artery Stenosis. Ultrasound Med Biol 2010; 36: 1973-1980

[302] Krogias C, Meves SH, Hansen C et al. Ultrasound Perfusion Imaging of the Brain - Routine and Novel Applications: Uncommon Cases and Review of the Literature. J Neuroimaging 2011; 21: 255-258

[303] Nedelmann M, Ritschel N, Doenges S et al. Combined Contrast-Enhanced Ultrasound and rt-PA Treatment is Safe and Improves Impaired Microcirculation after Reperfusion of Middle Cerebral Artery Occlusion. J Cereb Blood Flow Metab 2010; 30: 1712-1720

[304] Eggers ], Konig IR, Koch B et al. Sonothrombolysis With Transcranial Color-Coded Sonography and Recombinant Tissue-Type Plasminogen Activator in Acute Middle Cerebral Artery Main Stem Occlusion: Results From a Randomized Study. Stroke 2008; 39: 1470 - 1475

[305] Nacu A, Kvistad CE, Logallo $\mathrm{N}$ et al. A pragmatic approach to sonothrombolysis in acute ischaemic stroke: the Norwegian randomised controlled sonothrombolysis in acute stroke study (NOR-SASS). BMC Neurol 2015; 15: 110

[306] Schellinger PD, Alexandrov AV, Barreto AD et al. Combined Lysis of Thrombus with Ultrasound and Systemic Tissue Plasminogen Activator for Emergent Revascularization in Acute Ischemic Stroke (ClotbustER): Design and Methodology of a Multinational Phase 3 Trial. Int J Stroke 2015; 10: 1141 - 1148

[307] Saftoiu A, Dietrich CF, Vilmann P. Contrast-enhanced harmonic endoscopic ultrasound. Endoscopy 2012; 44: 612-617

[308] Alvarez-Sanchez MV, Napoleon B. Contrast-enhanced harmonic endoscopic ultrasound imaging: Basic principles, present situation and future perspectives. World J Gastroenterol 2014; 20: 15549-15563

[309] Fusaroli P, Napoleon B, Gincul R et al. The clinical impact of ultrasound contrast agents in EUS: a systematic review according to the levels of evidence. Gastrointest Endosc 2016; 84: 587-596

[310] Cosgrove D, Piscaglia F, Bamber JC et al. EFSUMB guidelines and recommendations on the clinical use of ultrasound elastography. Part 2: clinical applications. Ultraschall in Med 2013; 34: 238-253

[311] Jenssen C, Hocke M, Fusaroli P et al. EFSUMB Guidelines on Interventional Ultrasound (INVUS), Part IV: EUS-guided Interventions: General aspects and EUS-guided sampling (Long Version). Ultraschall in Med 2016; 37: E33 - E76

[312] Hocke M, Schulze E, Gottschalk P et al. Contrast-enhanced endoscopic ultrasound in discrimination between focal pancreatitis and pancreatic cancer. World J Gastroenterol 2006; 12: 246-250

[313] Gong T, Hu D, Zhu Q. Contrast-enhanced EUS for differential diagnosis of pancreatic mass lesions: a meta-analysis. Gastrointest Endosc 2012; 76: $301-309$
[314] Hocke M, Ignee A, Topalidis T et al. Contrast-Enhanced Endosonographic Doppler Spectrum Analysis Is Helpful in Discrimination Between Focal Chronic Pancreatitis and Pancreatic Cancer. Pancreas 2007; 35: $286-288$

[315] Hocke M, Ignee A, Dietrich CF. Advanced Endosonographic Diagnostic Tools for Discrimination of Focal Chronic Pancreatitis and Pancreatic Carcinoma - Elastography, Contrast Enhanced High Mechanical Index (CEHMI) and Low Mechanical Index (CELMI) Endosonography in Direct Comparison. Z Gastroenterol 2012; 50: 199-203

[316] Sakamoto H, Kitano M, Suetomi Y et al. Utility of Contrast-Enhanced Endoscopic Ultrasonography for Diagnosis of Small Pancreatic Carcinomas. Ultrasound Med Biol 2008; 34: 525-532

[317] Sâftoiu A, lordache S, Gheonea DI et al. Combined contrast-enhanced power Doppler and real-time sonoelastography performed during EUS, used in the differential diagnosis of focal pancreatic masses (with videos). Gastrointest Endosc 2010; 72: 739 - 747

[318] Fusaroli P, Spada A, Mancino MG et al. Contrast Harmonic Echo-Endoscopic Ultrasound Improves Accuracy in Diagnosis of Solid Pancreatic Masses. Clin Gastroenterol Hepatol 2010; 8: 629-634

[319] Gincul R, Palazzo M, Pujol B et al. Contrast-harmonic endoscopic ultrasound for the diagnosis of pancreatic adenocarcinoma: a prospective multicenter trial. Endoscopy 2014; 46: 373 - 379

[320] Napoleon B, Alvarez-Sanchez MV, Gincoul R et al. Contrast-enhanced harmonic endoscopic ultrasound in solid lesions of the pancreas: results of a pilot study. Endoscopy 2010; 42: $564-570$

[321] Sâftoiu A, Vilmann P, Dietrich CF et al. Quantitative contrast-enhanced harmonic EUS in differential diagnosis of focal pancreatic masses (with videos). Gastroint Endosc 2015; 82: 59-69

[322] Matsubara H, Itoh A, Kawashima $\mathrm{H}$ et al. Dynamic Quantitative Evaluation of Contrast-Enhanced Endoscopic Ultrasonography in the Diagnosis of Pancreatic Diseases. Pancreas 2011; 40: 1073-1079

[323] Kitano M, Kudo M, Yamao K et al. Characterization of Small Solid Tumors in the Pancreas: The Value of Contrast-Enhanced Harmonic Endoscopic Ultrasonography. Am J Gastroenterol 2012; 107: 303 - 310

[324] Lee TY, Cheon YK, Shim CS. Clinical Role of Contrast-Enhanced Harmonic Endoscopic Ultrasound in Differentiating Solid Lesions of the Pancreas: A Single-Center Experience in Korea. Gut Liver 2013; 7 : $599-604$

[325] Ishikawa T, Itoh A, Kawashima $\mathrm{H}$ et al. Usefulness of EUS combined with contrast-enhancement in the differential diagnosis of malignant versus benign and preoperative localization of pancreatic endocrine tumors. Gastrointest Endosc 2010; 71: 951 - 959

[326] Yamashita Y, Kato J, Ueda K et al. Contrast-Enhanced Endoscopic Ultrasonography for Pancreatic Tumors. Biomed Res Int 2015; 2015: 491782

[327] Seicean A, Badea R, Stan-luga R et al. Quantitative Contrast-Enhanced Harmonic Endoscopic Ultrasonography for the Discrimination of Solid Pancreatic Masses. Ultraschall in Med 2010; 31: 571 - 576

[328] Gheonea DI, Streba CT, Ciurea T et al. Quantitative low mechanical index contrast-enhanced endoscopic ultrasound for the differential diagnosis of chronic pseudotumoral pancreatitis and pancreatic cancer. BMC Gastroenterol 2013; 13: 2

[329] Imazu H, Kanazawa K, Mori N et al. Novel quantitative perfusion analysis with contrast-enhanced harmonic EUS for differentiation of autoimmune pancreatitis from pancreatic carcinoma. Scand j Gastroenterol 2012; 47: $853-860$

[330] Dietrich CF, Sahai AV, D’Onofrio M et al. Differential diagnosis of smal solid pancreatic lesions. Gastrointest Endosc 2016; 84: 933 - 940

[331] Seicean A, Badea R, Moldovan-Pop A et al. Harmonic Contrast-Enhanced Endoscopic Ultrasonography for the Guidance of Fine-Needle Aspiration in Solid Pancreatic Masses. Ultraschall in Med 2017; 38: $174-182$ 
[332] Hou X, Jin Z, Xu C et al. Contrast-Enhanced Harmonic Endoscopic Ultrasound-Guided Fine-Needle Aspiration in the Diagnosis of Solid Pancreatic Lesions: A Retrospective Study. PLoS ONE 2015; 10: e0121236

[333] Imazu H, Uchiyama Y, Matsunaga K et al. Contrast-enhanced harmonic EUS with novel ultrasonographic contrast (Sonazoid) in the preoperative T-staging for pancreaticobiliary malignancies. Scand j Gastroenterol 2010; 45: $732-738$

[334] Hirooka Y, Goto H, Ito A et al. Contrast-enhanced endoscopic ultrasonography in pancreatic diseases: a preliminary study. Am J Gastroenterol 1998; 93: 632-635

[335] Hocke M, Cui XW, Domagk D et al. Pancreatic cystic lesions: The value of contrast-enhanced endoscopic ultrasound to influence the clinical pathway. Endosc Ultrasound 2014; 3: 123-130

[336] Fusaroli P, Serrani M, De Giorgio R et al. Contrast Harmonic-Endoscopic Ultrasound Is Useful to Identify Neoplastic Features of Pancreatic Cysts (With Videos). Pancreas 2016; 45: 265-268

[337] Kamata K, Kitano M, Omoto S et al. Contrast-enhanced harmonic endoscopic ultrasonography for differential diagnosis of pancreatic cysts. Endoscopy 2016; 48: 35 -41

[338] Kurihara N, Kawamoto H, Kobayashi Y et al. Vascular patterns in nodules of intraductal papillary mucinous neoplasms depicted under contrast-enhanced ultrasonography are helpful for evaluating malignant potential. Eur J Radiol 2012; 81: 66-70

[339] Yamashita Y, Ueda K, Itonaga M et al. Usefulness of Contrast-Enhanced Endoscopic Sonography for Discriminating Mural Nodules From Mucous Clots in Intraductal Papillary Mucinous Neoplasms: A SingleCenter Prospective Study. J Ultrasound Med 2013; 32: 61 - 68

[340] Harima H, Kaino S, Shinoda S et al. Differential diagnosis of benign and malignant branch duct intraductal papillary mucinous neoplasm using contrast-enhanced endoscopic ultrasonography. World J Gastroenterol 2015; 21: $6252-6260$

[341] Ohno E, Itoh A, Kawashima $\mathrm{H}$ et al. Malignant Transformation of Branch DuctGÇôType Intraductal Papillary Mucinous Neoplasms of the Pancreas Based on Contrast-Enhanced Endoscopic Ultrasonography Morphological Changes: Focus on Malignant Transformation of Intraductal Papillary Mucinous Neoplasm Itself. Pancreas 2012; 41: 855862

[342] Park CH, Chung M], Oh TG et al. Differential diagnosis between gallbladder adenomas and cholesterol polyps on contrast-enhanced harmonic endoscopic ultrasonography. Surg Endosc 2013; 27: 14141421

[343] Choi JH, Seo DW, Choi JH et al. Utility of contrast-enhanced harmonic EUS in the diagnosis of malignant gallbladder polyps (with videos). Gastrointest Endosc 2013; 78: 484-493

[344] Imazu H, Mori N, Kanazawa K et al. Contrast-Enhanced Harmonic Endoscopic Ultrasonography in the Differential Diagnosis of Gallbladder Wall Thickening. Dig Dis Sci 2014; 59: 1909-1916

[345] Sugimoto M, Takagi T, Konno N et al. The efficacy of contrast-enhanced harmonic endoscopic ultrasonography in diagnosing gallbladder cancer. Sci Rep 2016; 6: 25848

[346] Kojima S, Goto H, Hirooka Y et al. Differentiation of benign and malignant lymph nodes with contrast-enhanced echolymphography using endoscopy ultrasound-guided puncture. Hepatogastroenterology 2003; 50: $1285-1291$

[347] Hocke M, Menges M, Topalidis T et al. Contrast-enhanced endoscopic ultrasound in discrimination between benign and malignant mediastinal and abdominal lymph nodes. J Cancer Res Clin Oncol 2008; 134: $473-480$

[348] Miyata T, Kitano M, Omoto S et al. Contrast-enhanced harmonic endoscopic ultrasonography for assessment of lymph node metastases in pancreatobiliary carcinoma. World J Gastroenterol 2016; 22: 3381 3391
[349] Iordache S, Filip MM, Georgescu CV et al. Contrast-enhanced power Doppler endosonography and pathological assessment of vascularization in advanced gastric carcinomas - a feasibility study. Med Ultrason 2012; 14: 101 - 107

[350] Matsui S, Kudo M, Kitano M et al. Evaluation of the response to chemotherapy in advanced gastric cancer by contrast-enhanced harmonic EUS. Hepatogastroenterology 2015; 62: 595- 598

[351] Nomura N, Goto H, Niwa Y et al. Usefulness of contrast-enhanced EUS in the diagnosis of upper Gl tract diseases. Gastrointest Endosc 1999; 50: $555-560$

[352] Kannengiesser K, Mahlke R, Petersen F et al. Contrast-enhanced harmonic endoscopic ultrasound is able to discriminate benign submucosal lesions from gastrointestinal stromal tumors. Scand j Gastroenterol 2012; 47: 1515-1520

[353] Sakamoto H, Kitano M, Matsui S et al. Estimation of malignant potential of GI stromal tumors by contrast-enhanced harmonic EUS (with videos). Gastrointest Endosc 2011; 73: 227-237

[354] Yamashita Y, Kato J, Ueda K et al. Contrast-enhanced endoscopic ultrasonography can predict a higher malignant potential of gastrointestinal stromal tumors by visualizing newly formed vessels. J Clin Ultrasound 2015; 43: 89-97

[355] Zhao Y, Qian L, Li P et al. The diagnostic value of endoscopic ultrasonography and contrast-enhanced harmonic endoscopic ultrasonography in gastrointestinal stromal tumors. Endosc Ultrasound 2016; 5: $111-117$

[356] Paik WH, Choi JH, Seo DW et al. Clinical Usefulness With the Combination of Color Doppler and Contrast-enhanced Harmonic EUS for the Assessment of Visceral Vascular Diseases. J Clin Gastroenterol 2014; 48: $845-850$

[357] Sato T, Yamazaki K, Toyota J et al. Evaluation of hemodynamics in esophageal varices: Value of endoscopic color Doppler ultrasonography with a galactose-based contrast agent. Hepatology Research 2003; 25: 55-61

[358] Sato T, Yamazaki K, Toyota J et al. Perforating veins in recurrent esophageal varices evaluated by endoscopic color Doppler ultrasonography with a galactose-based contrast agent. J Gastroenterol 2004; 39: $422-428$

[359] Sato T, Yamazaki K, Toyota J et al. Evaluation of arterial blood flow in esophageal varices via endoscopic color Doppler ultrasonography with a galactose-based contrast agent. J Gastroenterol 2005; 40: 64-69

[360] Chiu WC, Cushing BM, Rodriguez A et al. Abdominal Injuries without Hemoperitoneum: A Potential Limitation of Focused Abdominal Sonography for Trauma (FAST). J Trauma 1997; 42: 617-623

[361] Catalano O, Aiani L, Barozzi L et al. CEUS in abdominal trauma: multicenter study. Abdominal Imaging 2009; 34: 225 -234

[362] Valentino M, Serra C, Zironi G et al. Blunt Abdominal Trauma: Emergency Contrast-Enhanced Sonography for Detection of Solid Organ Injuries. Am J Roentgenol 2006; 186: 1361 - 1367

[363] Miele V, Piccolo CL, Galluzzo M et al. Contrast-enhanced ultrasound (CEUS) in blunt abdominal trauma. Br J Radiol 2016; 89: 20150823

[364] Sessa B, Trinci M, lanniello S et al. Blunt abdominal trauma: role of contrast-enhanced ultrasound (CEUS) in the detection and staging of abdominal traumatic lesions compared to US and CE-MDCT. Radiol Med 2015; 120: 180-189

[365] Cokkinos DD, Antypa E, Stefanidis K et al. Contrast-enhanced ultrasound for imaging blunt abdominal trauma - indications, description of the technique and imaging review. Ultraschall in Med 2012; 33: 60 67

[366] Durkin N, Deganello A, Sellars ME et al. Post-traumatic liver and spleen pseudoaneurysms in children: diagnosis, management and follow-up screening using contrast enhanced ultrasound (CEUS). J Pediat Surg 2016; $51: 289-292$ 
[367] Ferlay J, Steliarova-Foucher E, Lortet-Tieulent ] et al. Cancer incidence and mortality patterns in Europe: Estimates for 40 countries in 2012. Eur J Cancer 2013; 49: 1374-1403

[368] Haugen BR, Alexander EK, Bible KC et al. 2015 American Thyroid Association Management Guidelines for Adult Patients with Thyroid Nodules and Differentiated Thyroid Cancer: The American Thyroid Association Guidelines Task Force on Thyroid Nodules and Differentiated Thyroid Cancer. Thyroid 2015; 26: 1-133

[369] Bartolotta TV, Midiri M, Galia M et al. Qualitative and quantitative evaluation of solitary thyroid nodules with contrast-enhanced ultrasound: initial results. Eur Radiol 2006; 16: 2234-2241

[370] Zhang B, Jiang YX, Liu JB et al. Utility of Contrast-Enhanced Ultrasound for Evaluation of Thyroid Nodules. Thyroid 2010; 20: 51 - 57

[371] Yuan Z, Quan J, Yunxiao Z et al. Contrast-enhanced ultrasound in the diagnosis of solitary thyroid nodules. J Cancer Res Ther 2015; 11: 41 45

[372] Deng J, Zhou P, Tian S et al. Comparison of Diagnostic Efficacy of Contrast-Enhanced Ultrasound, Acoustic Radiation Force Impulse Imaging, and Their Combined Use in Differentiating Focal Solid Thyroid Nodules. PLoS ONE 2014; 9: e90674

[373] Nemec U, Nemec SF, Novotny C et al. Quantitative evaluation of contrast-enhanced ultrasound after intravenous administration of a microbubble contrast agent for differentiation of benign and malignant thyroid nodules: assessment of diagnostic accuracy. Eur Radiol 2012; 22: $1357-1365$

[374] Cantisani V, Consorti F, Guerrisi A et al. Prospective comparative evaluation of quantitative-elastosonography (Q-elastography) and contrast-enhanced ultrasound for the evaluation of thyroid nodules: Preliminary experience. Eur J Radiol 2013; 82: $1892-1898$

[375] Wu Q, Wang Y, Li Y et al. Diagnostic value of contrast-enhanced ultrasound in solid thyroid nodules with and without enhancement. Endocrine 2016; $1: 1-9$

[376] Ma X, Zhang B, Ling W et al. Contrast-enhanced sonography for the identification of benign and malignant thyroid nodules: Systematic review and meta-analysis. J Clin Ultrasound 2016; 44: 199-209

[377] Yu D, Han Y, Chen T. Contrast-Enhanced Ultrasound for Differentiation of Benign and Malignant Thyroid Lesions: Meta-analysis. Otolaryngol Head and Neck Surgery 2014; 151: 909 -915

[378] Sun B, Lang L, Zhu X et al. Accuracy of contrast-enhanced ultrasound in the identification of thyroid nodules: a meta-analysis. Int J Clin Exp Med 2015; 8: $12882-12889$

[379] Zhang XF, Hong YR, Bao XF et al. Qualitative evaluation of thyroid nodues with gray-scale contrast-enhanced ultrasound. Zhejiang Da Xue Xue Bao Yi Xue Ban 2008; 37: 515-518

[380] Acharya UR, Sree SV, Swapna G et al. Effect of complex wavelet transform filter on thyroid tumor classification in three-dimensional ultrasound. Proc Inst Mech Eng H 2013; 227: 284-292

[381] Vassallo P, Wernecke K, Roos N et al. Differentiation of benign from malignant superficial lymphadenopathy: the role of high resolution US. Radiology 1992; 183: 215-220

[382] Na DG, Lim HK, Byun HS et al. Differential diagnosis of cervical lymphadenopathy: usefulness of color Doppler sonography. Am J Roentgenol 1997; 168: $1311-1316$

[383] Schmid-Wendter MH, Partscht K, Korting HC et al. Improved differentiation of benign and malignant lymphadenopathy in patients with cutaneous melanoma by contrast-enhanced color Doppler sonography. Arc Dermatol 2002; 138: 491 -497

[384] Jin Y, He YS, Zhang MM et al. Value of contrast-enhanced ultrasonography in the differential diagnosis of enlarged lymph nodes: a metaanalysis of diagnostic accuracy studies. Asian Pac J Cancer Prev 2015; 16: $2361-2368$
[385] Moritz JD, Ludwig A, Oestmann JW. Contrast-enhanced color Doppler sonography for evaluation of enlarged cervical lymph nodes in head and neck tumors. Am J Roentgenol 2000; 174: 1279-1284

[386] Steppan I, Reimer D, Müller-Holzner E et al. Breast Cancer in Women: Evaluation of Benign and Malignant Axillary Lymph Nodes with Contrast-Enhanced Ultrasound. Ultraschall in Med 2010; 31: 63-67

[387] Stramare R, Scagliori E, Mannucci M et al. The Role of Contrast-Enhanced Gray-Scale Ultrasonography in the Differential Diagnosis of Superficial Lymph Nodes. Ultrasound Q 2010; 26: 45 - 51

[388] Rubaltelli L, Beltrame V, Tregnaghi A et al. Contrast-Enhanced Ultrasound for Characterizing Lymph Nodes With Focal Cortical Thickening in Patients With Cutaneous Melanoma. Am J Roentgenol 2011; 196 : W8-W12

[389] Dudau C, Hameed S, Gibson D et al. Can Contrast-Enhanced Ultrasound Distinguish Malignant from Reactive Lymph Nodes in Patients with Head and Neck Cancers? Ultrasound Med Biol 2014; 40: 747 - 754

[390] Yu M, Liu Q, Song HP et al. Clinical Application of Contrast-Enhanced Ultrasonography in Diagnosis of Superficial Lymphadenopathy. J Ultrasound Med 2010; 29: 735 - 740

[391] Nakase K, Yamamoto K, Hiasa A et al. Contrast-enhanced ultrasound examination of lymph nodes in different types of lymphoma. Cancer Detect Prev 2006; 30: 188-191

[392] Rubaltelli L, Khadivi Y, Tregnaghi A et al. Evaluation of Lymph Node Perfusion Using Continuous Mode Harmonic Ultrasonography With a Second-Generation Contrast Agent. J Ultrasound Med 2004; 23: 829 836

[393] Jin Y, Peng YL, Ma BY et al. The perfusion pattern of lymphadenopathy in contrast-enhanced ultrasonography under different reference conditions. Sichuan Da Xue Xue BAo Yi Xue Ban 2014; 45: 1005 - 1009

[394] Steinhart H, Zenk J, Sprang K et al. Contrast-enhanced color Doppler sonography of parotid gland tumors. Eur Arch OtorhinoLaryngol 2003 260: $344-348$

[395] Mansour N, Bas M, Stock KF et al. Multimodal Ultrasonographic Pathway of Parotid Gland Lesions. Ultraschall in Med 2017; 38: 166-173

[396] Knopf A, Mansour N, Chaker A et al. Multimodal ultrasonographic characterisation of parotid gland lesions\&\#x2014;A pilot study. Eur J Radiol 2012; 81: 3300-3305

[397] Wei X, Li Y, Zhang S et al. Evalution of microvascularization in focal salivary gland lesions by contrast-enhanced ultrasonography (CEUS) and color Doppler sonography. Clin Hemorheol Microcirc 2013; 54 : $259-271$

[398] Klotz LV, Gorkov R, Eichhorn ME et al. Perfusion characteristics of parotid gland tumors evaluated by contrast-enhanced ultrasound. Eur ] Radiol 2013; 82: $2227-2232$

[399] David E, Cantisani V, De Vincentiis M et al. Contrast-enhanced ultrasound in the evaluation of parotid gland lesions: an update of the literature. Ultrasound 2016; 24: $104-110$

[400] Badea AF, Bran S, Tamas-Szora A et al. Solid parotid tumors: an individual and integrative analysis of various ultrasonographic criteria. A prospective and observational study. Med Ultrason 2013; 15: 289 298

[401] Klotz LV, Ingrisch M, Eichhorn ME et al. Monitoring parotid gland tumors with a new perfusion software for contrast-enhanced ultrasound. Clin Hemorheol Microcirc 2014; 58: 261 - 269

[402] Fischer T, Paschen CF, Slowinski T et al. Differentiation of Parotid Gland Tumors with Contrast-Enhanced Ultrasound. Rofo 2010; 182: 155 162

[403] Sorelli PG, Cosgrove DO, Svensson WE et al. Can contrast-enhanced sonography distingush benign from malignant breast masses. J Clin Ultrasound 2010; 38: 177 - 181 
[404] Balleyguier C, Opolon P, Mathieu MC et al. New potential and applications of contrast-enhanced ultrasound of the breast: Own investigations and review of the literature. Eur J Radiol 2009; 69: 14-23

[405] Liu H, Jiang YX, Liu JB et al. Evaluation of breast lesions with contrastenhanced ultrasound using the microvascular imaging technique: Initial observations. Breast 2008; 17: $532-539$

[406] Jia WR, Chai WM, Tang L et al. Three-dimensional contrast enhanced ultrasound score and dynamic contrast-enhanced magnetic resonance imaging score in evaluating breast tumor angiogenesis: Correlation with biological factors. Eur J Radiol 2014; 83: $1098-1105$

[407] Cao XL, Bao W, Zhu SG et al. Contrast-Enhanced Ultrasound Characteristics of Breast Cancer: Correlation with Prognostic Factors. Ultrasound Med Biol 2014; 40: 11-17

[408] Zhang J, Cai L, Chen L et al. CEUS Helps to Rerate Small Breast Tumors of BI-RADS Category 3 and Category 4. Biomed Res Int 2014; 2014: 572532

[409] Xia HS, Wang X, Ding H et al. Papillary breast lesions on contrast-enhanced ultrasound: morphological enhancement patterns and diagnostic strategy. Eur Radiol 2014; 24: 3178-3190

[410] Wang Z, Zhou Q, Liu J et al. Tumor size of breast invasive ductal cancer measured with contrast-enhanced ultrasound predicts regional lymph node metastasis and N stage. Int J Clin Exp Pathol 2014; 7: 6985-6991

[411] Omoto K, Matsunaga $\mathrm{H}$, Take $\mathrm{N}$ et al. Sentinel Node Detection Method Using Contrast-Enhanced Ultrasonography with Sonazoid in Breast Cancer: Preliminary Clinical Study. Ultrasound Med Biol 2009; 35: $1249-1256$

[412] Sever A, Jones S, Cox K et al. Preoperative localization of sentinel lymph nodes using intradermal microbubbles and contrast-enhanced ultrasonography in patients with breast cancer. Br J Surg 2009; 96: 1295 1299

[413] Sever AR, Mills P, Weeks J et al. Preoperative Needle Biopsy of Sentinel Lymph Nodes Using Intradermal Microbubbles and Contrast-Enhanced Ultrasound in Patients With Breast Cancer. Am J Roentgenol 2012; 199: $465-470$

[414] Sever AR, Mills P, Jones SE et al. Preoperative sentinel node identification with ultrasound using microbubbles in patients with breast cancer. Am J Roentgenol 2011; 196: 251 - 256

[415] Cox K, Sever A, Jones S et al. Validation of a technique using microbubbles and contrast enhanced ultrasound (CEUS) to biopsy sentinel lymph nodes (SLN) in pre-operative breast cancer patients with a normal grey-scale axillary ultrasound. Eur J Surg Oncol 2013; 39: 760 - 765

[416] Wang Y, Wang W, Li J et al. Gray-Scale Contrast-Enhanced Ultrasonography Of Sentinel Lymph Nodes In A Metastatic. Acad Radiol 2009; 16: $957-962$

[417] Xie F, Zhang D, Cheng L et al. Intradermal microbubbles and contrastenhanced ultrasound (CEUS) is a feasible approach for sentinel lymph node identification in early-stage breast cancer. World J Surg Oncol 2015; 13: 319

[418] Gkegkes ID, lavazzo C. Contrast enhanced ultrasound (CEU) using microbubbles for sentinel lymph node biopsy in breas cancer; a systemic review. Acta Chir Belg 2015; 115: 212-218

[419] FitzGerald O, Bresnihan B. Synovial membrane cellularity and vascularity. Ann Rheum Dis 1995; 54: 511- 515

[420] Klauser A, Frauscher F, Schirmer M. Letter to the Editor. Eur Radiol 2004; 14: 545- 546

[421] Klauser AS, Peetrons P. Developments in musculoskeletal ultrasound and clinical applications. Skeletal Radiol 2010; 39: 1061-1071

[422] De Zordo T, Mlekusch SP, Feuchtner GM et al. Value of contrast-enhanced ultrasound in rheumatoid arthritis. Eur J Radiol 2007; 64: 222 230

[423] Taylor PC. The value of sensitive imaging modalities in rheumatoid arthritis. Arthritis Res Ther 2003; 5: 210-213
[424] Hermann KG, Backhaus M, Schneider U et al. Rheumatoid arthritis of the shoulder joint: Comparison of conventional radiography, ultrasound, and dynamic contrast-enhanced magnetic resonance imaging. Arthritis Rheum 2003; 48: 3338-3349

[425] Klauser A, Frauscher F, Schirmer M et al. The value of contrast-enhanced color doppler ultrasound in the detection of vascularization of finger joints in patients with rheumatoid arthritis. Arthritis Rheum 2002; 46: 647-653

[426] Fiocco U, Stramare R, Coran A et al. Vascular perfusion kinetics by contrast-enhanced ultrasound are related to synovial microvascularity in the joints of psoriatic arthritis. Clin Rheumatol 2015; 34: 1903 1912

[427] Doria SA, Kiss BMH, Lotito NAP et al. Juvenile rheumatoid arthritis of the knee: evaluation with contrast-enhanced color Doppler ultrasound. Pediatr Radiol 2001; 31: 524-531

[428] Klauser A, Halpern E], Frauscher $F$ et al. Inflammatory low back pain: High negative predictive value of contrast-enhanced color Doppler ultrasound in the detection of inflamed sacroiliac joints. Arthritis Rheum 2005; 53: 440-444

[429] Magarelli N, Guglielmi G, Di Matteo L et al. Diagnostic utility of an echo-contrast agent in patients with synovitis using power Doppler ultrasound: a preliminary study with comparison to contrast-enhanced MRI. Eur Radiol 2001; 11: 1039-1046

[430] Klauser A, Demharter J, De Marchi A et al. Contrast enhanced grayscale sonography in assessment of joint vascularity in rheumatoid arthritis: results from the IACUS study group. Eur Radiol 2005; 15: $2404-2410$

[431] Schueller-Weidekamm C, Krestan C, Schueller G et al. Power Doppler Sonography and Pulse-Inversion Harmonic Imaging in Evaluation of Rheumatoid Arthritis Synovitis. Am J Roentgenol 2007; 188: 504-508

[432] Cai XH, Yang SP, Shen HL et al. Application of contrast-enhanced ultrasonography and ultrasonography scores in rheumatoid arthritis. Int J Clin Exp Med 2015; 8: 20056-20064

[433] Klauser AS, Franz M, Arora R et al. Detection of vascularity in wrist tenosynovitis: power doppler ultrasound compared with contrast-enhanced grey-scale ultrasound. Arthritis Res Ther 2010; 12: R209

[434] Wamser G, Bohndorf K, Vollert K et al. Power Doppler sonography with and without echo-enhancing contrast agent and contrast-enhanced MRI for the evaluation of rheumatoid arthritis of the shoulder joint: differentiation between synovitis and joint effusion. Skeletal Radiol 2003; 32: 351-359

[435] Carotti M, Salaffi F, Manganelli P et al. Power Doppler sonography in the assessment of synovial tissue of the knee joint in rheumatoid arthritis: a preliminary experience. Ann Rheum Dis 2002; 61: 877-882

[436] Hau M, Kneitz C, Tony H et al. High resolution ultrasound detects a decrease in pannus vascularisation of small finger joints in patients with rheumatoid arthritis receiving treatment with soluble tumour necrosis factor alpha receptor (etanercept). Ann Rheum Dis 2002; 61: $55-58$

[437] Genovese E, Ronga M, Recaldini C et al. Analysis of achilles tendon vascularity with second-generation contrast-enhanced ultrasound. J Clin Ultrasound 2011; 39: 141 - 145

[438] Song IH, Althoff CE, Hermann KG et al. Contrast-enhanced ultrasound in monitoring the efficacy of a bradykinin receptor 2 antagonist in painful knee osteoarthritis compared with MRI. Ann Rheum Dis 2009; 68: $75-83$

[439] Qvistgaard E, Rogind H, Torp-Pedersen S et al. Quantitative ultrasonography in rheumatoid arthritis: evaluation of inflammation by Doppler technique. Ann Rheum Dis 2001; 60: 690-693

[440] Stramare R, Raffeiner B, Ciprian L et al. Evaluation of finger joint synovial vascularity in patients with rheumatoid arthritis using contrastenhanced ultrasound with water immersion and a stabilized probe. J Clin Ultrasound 2012; 40: 147-154 
[441] Xie XH, Xu HX, Xie XY et al. Differential diagnosis between benign and malignant gallbladder diseases with real-time contrast-enhanced ultrasound. Eur Radiol 2010; 20: 239-248

[442] Tang S, Wang Y, Wang Y. Contrast-enhanced ultrasonography to diagnose gallbladder perforation. Am J Emerg Med 2013; 31: 1240 1243

[443] Fei X, Lu WP, Luo YK et al. Contrast-enhanced ultrasound may distinguish gallbladder adenoma from cholesterol polyps: a prospective caseGÇôcontrol study. Abdom Imaging 2015; 40: 2355 - 2363

[444] Sun LP, Guo LH, Xu HX et al. Value of contrast-enhanced ultrasound in the differential diagnosis between gallbladder adenoma and gallbladder adenoma canceration. Int J Clin Exp Med 2015; 8: 1115-1121

[445] Liu LN, Xu HX, Lu MD et al. Contrast-Enhanced Ultrasound in the Diagnosis of Gallbladder Diseases: A Multi-Center Experience. PLoS ONE 2012; 7: e48371

[446] Date RS, Thrumurthy SG, Whiteside S et al. Gallbladder perforation: Case series and systematic review. Int J Surg 2012; 10: 63-68

[447] Smith EA, Dillman JR, Elsayes KM et al. Cross-Sectional Imaging of Acute and Chronic Gallbladder Inflammatory Disease. Am J Roentgenol 2009; 192: 188-196

[448] Kawai R, Hata J, Manabe N et al. Contrast-enhanced ultrasonography with Sonazoid for diagnosis of gangrenous cholecystitis. J Med Ultrason 2016; 43: $193-199$

[449] Revel L, Lubrano J, Badet $\mathrm{N}$ et al. Preoperative diagnosis of gangrenous acute cholecystitis: usefulness of CEUS. Abdom Imaging 2014; 39 : $1175-1181$

[450] Ripolles T, Martinez-Perez M], Martin G et al. Usefulness of contrastenhanced US in the diagnosis of acute gangrenous cholecystitis: $A$ comparative study with surgical and pathological findings. Eur J Radiol 2016; 85: $31-38$

[451] Adamietz B, Wenkel E, Uder M et al. Contrast enhanced sonography of the gallbladder: A tool in the diagnosis of cholecystitis? Eur J Radiol 2007; 61: $262-266$

[452] Shapira-Rootman M, Mahamid A, Reindorp N et al. Diagnosis of gallbladder perforation by ultrasound. Clin Imaging 2015; 39: 827-829

[453] Sagrini E, Pecorelli A, Pettinari I et al. Contrast-enhanced ultrasonography to diagnose complicated acute cholecystitis. Intern Emerg Med 2016; 11: 19-30

[454] Runner G], Corwin MT, Siewert B et al. Gallbladder Wall Thickening. Am J Roentgenol 2013; 202: W1 - W12

[455] Xu JM, Guo LH, Xu HX et al. Differential Diagnosis of Gallbladder Wall Thickening: The-áUsefulness of Contrast-Enhanced Ultrasound. Ultrasound Med Biol 2014; 40: 2794-2804

[456] Mellnick VM, Menias CO, Sandrasegaran K et al. Polypoid Lesions of the Gallbladder: Disease Spectrum with Pathologic Correlation. Radiographics 2015; 35: 387-399

[457] Gallahan WC, Conway JD. Diagnosis and Management of Gallbladder Polyps. Gastroenterol Clin North Am 2010; 39: 359-367

[458] Corwin MT, Siewert B, Sheiman RG et al. Incidentally Detected Gallbladder Polyps: Is Follow-up Necessary?GÇöLong-term Clinical and US Analysis of 346 Patients. Radiology 2011; 258: 277-282

[459] Aloia TA, J+írufe N, Javle M et al. Gallbladder Cancer: expert consensus statement. HPB (Oxford) 2015; 17: 681-690

[460] Yuan HX, Cao JY, Kong WT et al. Contrast-enhanced ultrasound in diagnosis of gallbladder adenoma. Hepatobiliary Pancreat Dis Int 2015; 14: $201-207$

[461] Nabatame N, Shirai Y, Nishimura A et al. High risk of gallbladder carcinoma in elderly patients with segmental adenomyomatosis of the gallbladder. J Exp Clin Cancer Res 2004; 23: 593 - 598

[462] Tang S, Huang L, Wang Y et al. Contrast-enhanced ultrasonography diagnosis of fundal localized type of gallbladder adenomyomatosis. BMC Gastroenterol 2015; 15: 99
[463] Levy AD, Murakata LA, Rohrmann CA. Gallbladder Carcinoma: Radiologic-Pathologic Correlation. Radiographics 2001; 21: 295 - 314

[464] Stinton LM, Shaffer EA. Epidemiology of Gallbladder Disease: Cholelithiasis and Cancer. Gut Liver 2012; 6: 172-187

[465] Meacock LM, Sellars ME, Sidhu PS. Evaluation of Gall Bladder and Biliary Duct Disease Using Microbubble Contrast Enhanced Ultrasound. $\mathrm{Br}$ ] Radiol 2010; 83: 615-627

[466] Yoon WJ, Yoon YB, Kim Y] et al. Metastasis to the gallbladder: A singlecenter experience of 20 cases in South Korea. World J Gastroenterol 2009; 15: $4806-4809$

[467] Barretta ML, Catalano O, Setola SV et al. Gallbladder metastasis: spectrum of imaging findings. Abdom Imaging 2011; 36: 729-734

[468] Engelhardt M, Hansen C, Eyding J et al. Feasibility of Contrast-Enhanced Sonography During Resection of Cerebral Tumours: Initial Results of a Prospective Study. Ultrasound Med Biol 2007; 33: 571-575

[469] He W, Jiang X, Wang $S$ et al. Intraoperative contrast-enhanced ultrasound for brain tumors. Clin Imaging 2008; 32: 419-424

[470] Holscher T, Ozgur B, Singel S et al. Introoperative ultrasound using phase inversion harmonic imaging: first experiences. Neurosurgery 2007; 60: $382-386$

[471] Kanno H, Ozawa Y, Sakata K et al. Intraoperative power Doppler ultrasonography with a contrast-enhancing agent for intracranial tumors. J Neurosurg 2005; 102: 295-301

[472] Prada F, Del Bene M, Moiraghi A et al. From Grey Scale B-Mode to Elastosonography: Multimodal Ultrasound Imaging in Meningioma SurgeryGÇöPictorial Essay and Literature Review. Biomed Res Int 2015; 2015: 925729

[473] Prada F, Del Bene M, Saini M et al. Intraoperative cerebral angiosonography with ultrasound contrast agents: how I do it. Acta Neurochir (Wein) 2015; 157: 1025-1029

[474] Prada F, Perin A, Martegani A et al. Intraoperative Contrast-Enhanced Ultrasound for Brain Tumor Surgery. Neurosurgery 2014; 74: 542 - 552

[475] He W, Wang L, Li HZ et al. Intraoperative contrast-enhanced ultrasound in traumatic brain surgery. Clin Imaging 2013; 37: 983 - 988

[476] Arlt F, Chalopin C, Muns A et al. Intraoperative 3D contrast-enhanced ultrasound (CEUS): a prospective study of 50 patients with brain tumours. Acta Neurochir (Wein) 2016; 158: 685-694

[477] Prada F, Bene MD, Fornaro R et al. Identification of residual tumor with intraoperative contrast-enhanced ultrasound during glioblastoma resection. Neurosurg Focus 2016; 40: E7

[478] Lekht I, Brauner N, Bakhsheshian J et al. Versatile utilization of realtime intraoperative contrast-enhanced ultrasound in cranial neurosurgery: technical note and retrospective case series. Neurosurg Focus 2016; 40: E6

[479] Prada F, Mattei L, Del Bene M et al. Intraoperative Cerebral Glioma Characterization with Contrast Enhanced Ultrasound. Biomed Res Int 2014; 2014: 484261

[480] Prada F, Bene MD, Casali C et al. Intraoperative Navigated Angiosonography for Skull Base Tumor Surgery. World Neurosurg 2015; 84: $1699-1707$

[481] Huang DY, Yusuf GT, Daneshi M et al. Contrast-enhanced US guided Interventions: Improving Success Rate and Avoiding Complications Using US Contrast Agents. Radiographics 2016; 37: 652 - 664

[482] Bang N, Bachmann Nielsen M, Vejborg I et al. Clinical report: contrast enhancement of tumor perfusion as a guidance for biopsy. Eur J Ultrasound 2000; 12: 159-161

[483] Wu W, Chen MH, Yin SS et al. The Role of Contrast-Enhanced Sonography of Focal Liver Lesions Before Percutaneous Biopsy. Am J Roentgenol 2006; 187: 752 - 761

[484] Wang S, Yang W, Zhang $\mathrm{H}$ et al. The Role of Contrast-Enhanced Ultrasound in Selection Indication and Improveing Diagnosis for Transthor- 
acic Biopsy in Peripheral Pulmonary and Mediastinal Lesions. Biomed Res Int 2015; 2015: 231782

[485] Cao BS, Wu JH, Li XL et al. Sonographically Guided Transthoracic Biopsy of Peripheral Lung and Mediastinal Lesions: Role of Contrast-Enhanced Sonography. J Ultrasound Med 2011; 30: 1479-1490

[486] Sparchez Z, Radu P, Kasco G et al. Contrast-enhanced ultrasound guided biopsy of superficial toraco-abdominal and neck lesions. Initial experience in 20 patients. Med Ultrason 2012; 14: 288-293

[487] De Marchi A, Brach del Prever EM, Linari A et al. Accuracy of core-needle biopsy after contrast-enhanced ultrasound in soft-tissue tumours. Eur Radiol 2010; 20: 2740 - 2748

[488] Loizides A, Widmann G, Freuis T et al. Optimizing Ultrasound-Guided Biopsy of Musculoskeletal Masses by Application of an Ultrasound Contrast Agent. Ultraschall in Med 2011; 32: 307-310

[489] Schlottmann K, Klebl F, Zorger N et al. Contrast-Enhanced Ultrasound Allows for-álnterventions of Hepatic Lesions which are Invisible onConventional B-Mode. Z Gastroenterol 2004; 42: 303-310

[490] Yoon SH, Lee KH, Kim SY et al. Real-time contrast-enhanced ultrasound-guided biopsy of focal hepatic lesions not localised on B-mode ultrasound. Eur Radiol 2010; 20: 2047 - 2056

[491] Tang J, Zhang H, Lv F et al. Percutaneous Injection Therapy for Blunt Splenic Trauma Guided by Contrast-Enhanced Ultrasonography. J Ultrasound Med 2008; 27: 925-932

[492] Tokunaga S, Koda M, Kato J et al. Assessment of Track Microbubble Flow Signals on Contrast-enhanced Ultrasound with Perflubutane Following Percutaneous Liver Biopsy. Intern Med 2013; 52: 1455 - 1459

[493] Lorentzen T, Nolsoe CP, Ewertsen C et al. EFSUMB guidelines on interventional ultrasound (INVUS), Part I general aspects (short version). Ultraschall in Med 2015; 36: 461 - 472

[494] Wink MH, Lagerveld BW, Laguna MP et al. Cryotherapy for Renal-Cell Cancer: Diagnosis, Treatment, and Contrast-Enhanced Ultrasonography for Follow-Up. J Endourol 2006; 20: 456-459

[495] Johnson DB, Duchene DA, Taylor GD et al. Contrast-Enhanced Ultrasound Evaluation of Radiofrequency Ablation of the Kidney: Reliable Imaging of the Thermolesion. J Endourol 2005; 19: 248-252

[496] Campbell SC, Novick AC, Belldegrun A et al. Guideline for Management of the Clinical T1 Renal Mass. J Urol 2009; 182: 1271-1279

[497] Ignee A, Straub B, Schuessler G et al. Contrast enhanced ultrasound of renal masses. World ] Radiol 2010; 2: 15-31

[498] Hoeffel C, Pousset M, Timsit MO et al. Radiofrequency ablation of renal tumours: diagnostic accuracy of contrast-enhanced ultrasound for early detection of residual tumour. Eur Radiol 2010; 20: 1812-1821

[499] Meloni MF, Bertolotto M, Alberzoni C et al. Follow-Up After Percutaneous Radiofrequency Ablation of Renal Cell Carcinoma: Contrast-Enhanced Sonography Versus Contrast-Enhanced CT or MRI. Am J Roentgenol 2008; 191: $1233-1238$

[500] Meloni MF, Smolock A, Cantisani V et al. Contrast enhanced ultrasound in the evaluation and percutaneous treatment of hepatic and renal tumors. Eur J Radiol 2015; 84: 1666-1674

[501] Oh TH, Lee YH, Seo IY. Diagnostic Efficacy of Contrast-Enhanced Ultrasound for Small Renal Masses. Korean J Urol 2014; 55: 587 - 592

[502] Ascenti G, Gaeta M, Magno C et al. Contrast-Enhanced Second-Harmonic Sonography in the Detection of Pseudocapsule in Renal Cell Carcinoma. Am J Roentgenol 2004; 182: 1525-1530

[503] Li X, Liang P, Yu J et al. Role of contrast-enhanced ultrasound in evaluating the efficiency of ultrasound guided percutaneous microwave ablation in patients with renal cell carcinoma. Radiol Oncol 2013; 47: $398-404$

[504] Kong WT, Zhang WW, Guo HQ et al. Application of contrast-enhanced ultrasonography after radiofrequency ablation for renal cell carcinoma: is it sufficient for assessment of therapeutic response? Abdom Imaging 2011; 36: $342-347$
[505] Zhao X, Wang W, Zhang S et al. Improved outcome of percutaneous radiofrequency ablation in renal cell carcinoma: a retrospective study of intraoperative contrast-enhanced ultrasonography in 73 patients. Abdom Imaging 2012; 37: 885-891

[506] Barwari K, Wijkstra H, van Delden OM et al. Contrast-Enhanced Ultrasound for the Evaluation of the Cryolesion After Laparoscopic Renal Cryoablation: An Initial Report. J Endourol 2012; 27: $402-407$

[507] Murat FJ, Poissonnier L, Rabilloud M et al. Mid-term Results Demonstrate Salvage High-Intensity Focused Ultrasound (HIFU) as an Effective and Acceptably Morbid Salvage Treatment Option for Locally Radiorecurrent Prostate Cancer. Eur Urol 2009; 55: 640 - 649

[508] Rouviere O, Glas L, Girouin N et al. Prostate Cancer Ablation with Transrectal High-Intensity Focused Ultrasound: Assessment of Tissue Destruction with Contrast-enhanced US. Radiology 2011; 259: 583 591

[509] Lei F, Jing Z, Bo W et al. Uterine myomas treated with microwave ablation: The agreement between ablation volumes obtained from contrast-enhanced sonography and enhanced MRI. Int J Hyperthermia 2014; 30: $11-18$

[510] Orsi F, Monfardini L, Bonomo G et al. Ultrasound guided high intensity focused ultrasound (USgHIFU) ablation for uterine fibroids: Do we need the microbubbles? Int J Hyperthermia 2015; 31: 233 - 239

[511] Peng S, Hu L, Chen W et al. Intraprocedure contrast enhanced ultrasound: The value in assessing the effect of ultrasound-guided high intensity focused ultrasound ablation for uterine fibroids. Ultrasonics 2015; 58: $123-128$

[512] Wang F, Zhang J, Han ZY et al. Imaging manifestation of conventional and contrast-enhanced ultrasonography in percutaneous microwave ablation for the treatment of uterine fibroids. Eur J Radiol 2012; 81: $2947-2952$

[513] Ignee A, Jenssen C, Cui XW et al. Intracavitary contrast-enhanced ultrasound in abscess drainage - feasibility and clinical value. Scand J Gastroenterol 2016; 51: 41 - 47

[514] Lanzani C, Savasi V, Leone FP et al. Two-dimensional HyCoSy with contrast tuned imaging technology and a second generation contrast media for the assessment of tubal patency in an infertility program. Fertil-Steril 2009; 92: 1158-1161

[515] Boudghene FP, BAzot M, Robert Y et al. Assessment of fallopian tube patency by HyCoSy: comparison of a positive contrast agent with saline solution. Ultrasound Obstet Gynecol 2001; 18: 525 - 530

[516] Tamasi F, Weidner A, Domokos N et al. ECHOVIST-200 enhanced hystero-sonography. Eur ] Obstet Gynecol Reprod Biol 2005; 121: 186 190

[517] Foschi FG, Piscaglia F, Pompili M et al. Real-Time Contrast-Enhanced Ultrasound GÇô a-áNew-áSimple Tool for Detection of PeritonealPleural Communications in Hepatic Hydrothorax. Ultraschall in Med 2008; 29: $538-542$

[518] Matono T, Koda M, Murawaki Y. Right diaphragmatic defect in hepatic hydrothorax exposed by contrast-enhanced ultrasonography after radiofrequency ablation. Hepatology 2016; 56: 784-785

[519] Tamano M, Hashimoto T, Kojima K et al. Diagnosis of hepatic hydrothorax using contrast-enhanced ultrasonography with intraperitoneal injection of Sonazoid. J Gastroenterol Hepatol 2010; 25: 383-386

[520] Ignee A, Baum U, Schuessler G et al. Contrast-enhanced ultrasoundguided percutaneous cholangiography and cholangiodrainage (CEUSPTCD). Endoscopy 2009; 41: 725-726

[521] Urade T, Fukumoto T, Tanaka M et al. Contrast-Enhanced Intraoperative Ultrasonic Cholangiography for Real-Time Biliary Navigation in Hepatobiliary Surgery. J Am Coll Surg 2014; 218: e43 - e50

[522] Chopra SS, Eisele R, Seehofer D et al. Contrast enhanced ultrasound cholangiography via T-tube following liver transplantation. Ann Transplant 2012; 17: $108-112$ 
[523] Daneshi M, Rajayogeswaran B, Peddu P et al. Demonstration of an ocult biliary-arterial fistula using percutaneous contrast-enhanced ultrasound cholangiography in a transplanted liver. J Clin Ultrasound 2014; 42: 108-111

[524] Mao R, Xu EJ, Li K et al. Usefulness of contrast-enhanced ultrasound in the diagnosis of biliary leakage following T-tube removal. J Clin Ultrasound 2010; 38: $38-40$

[525] Xu E], Mao R, Zheng RQ et al. Three-dimensional contrast-enhanced ultrasonic cholangiography: a new technique for delineation of the biliary tract in a liver donor. Liver Transpl 2009; 15: 1154-1156

[526] Zheng RQ, Chen G, Xu EJ et al. Evaluating Biliary Anatomy and Variations in Living Liver Donors by a New Technique: Three-Dimensional Contrast-Enhanced Ultrasonic Cholangiography. Ultrasound Med Biol 2010; 36: $1282-1287$

[527] Velosa M, Lopes S, Castro R et al. Cholecystoduodenal fistula diagnosed with contrast-enhanced endoscopic ultrasound. Endoscopy 2013; 45: E18-E19

[528] Zuber-Jerger I, Endlicher E, Schälmerich J et al. Endoscopic retrograde cholangiography with contrast ultrasonography. Endoscopy 2008; 40 (Suppl. 2): E202

[529] Cui XW, Ignee A, Maros T et al. Feasibility and Usefulness of Intra-cavitary Contrast-Enhanced Ultrasound in Percutaneous Nephrostomy. Ultrasound Med Biol 2016; 42: $2180-2188$

[530] Chi T, Usawachintachit M, Mongan J et al. Feasibility of Antegrade Contrast-enhanced US Nephrostograms to Evaluate Ureteral Patency. Radiology 2016; 283: 273 - 279

[531] Zengel P, Berghaus A, Weiler C et al. Intraductally applied contrastenhanced ultrasound (IA-CEUS) for evaluating obstructive disease and secretory dysfunction of the salivary glands. Eur Radiol 2011; 21: $1339-1348$

[532] Zengel P, Siedek V, Berghaus A et al. Intraductally applied contrastenhanced ultrasound (IA-CEUS) for improved visualization of obstructive diseases of the salivary glands, primary results. Clin Hemorheol Microcirc 2010; 45: 193-295

[533] Chew SS, Yang JL, Newstead GL et al. Analfistula: Levovist-enhanced endoanal ultrasound: a pilot study. Dis Colon Rectum 2003; 46: 377 384

[534] Chen Y], Mao R, Xie XH et al. Intracavitary Contrast-enhanced Ultrasonography to Detect Enterovesical Fistula in Crohns Disease. Gastroenterology 2016; 150: $315-317$

[535] Volkmer BG, Nesslauer T, Kufer R et al. Diagnostik von HarnblasenDarm-Fisteln durch kontrastmittelunterstetzte 3D-Sonographie. Ultraschall in Med 2001; 22: $81-86$

[536] Girlich C, Buttner R, Schacherer D et al. Kontrastmittelsonografische Drainagenkontrolle: eine Feasibility-Studie. Z Gastroenterol 2011; 49 : $1470-1474$

[537] Muller T, Blank W, Leitlein J et al. Endocavitary contrast-enhanced ultrasound: A technique whose time has come? J Clin Ultrasound 2015; 43: $71-80$

[538] Prantl L, Pfister K, Kubale R et al. Value of high resolution ultrasound and contrast enhanced US pulse inversion imaging for teh evaluation of the vascular integrity of free-flap grafts. Clin Hemorheol Microcirc 2007; 36: $203-216$

[539] Gardiner MD, Nanchahal J. Strategies to ensure success of microvascular free tissue transfer. J Plast Reconstr Aesthet Surg 2010; 63: e665-e673

[540] Prantl L, Schmitt S, Geis S et al. Contrast harmonic ultrasound and indocyanine-green fluorescence video angiography for evaluation of dermal and subdermal microcirculation in free parascapular flaps. Clin Hemorheol Microcirc 2016; 38: $105-118$

[541] Prantl L, Schremi S, Walter M et al. Evaluation of microcirculation of free flaps of the lower leg by contrast harmonic imaging (CHI) with time intensity curve (TIC) analysis. Clin Hemorheol Microcirc 2008; 39: $343-350$

[542] Geis S, Prantl L, Dolderer J et al. Postoperative Monitoring of Local and Free Flaps with Contrast-Enhanced Ultrasound (CEUS) - Analysis of 112 Patients. Ultraschall in Med 2013; 34: 550 - 558

[543] Jung EM, Prantl L, Schreyer AG et al. New perfusion imaging of tissue transplants with contrast harmonic ultrasound imaging $(\mathrm{CHI})$ and magnetic resonance imaging (MRI) in comparison with laser-induced indocyanine green (ICG) fluorescence angiography. Clin Hemorheol Microcirc 2009; 43: 19-33

[544] Geis S, Prantl L, Gehmert S et al. TTP (time to peak) and RBV (regional blood volume) as valuable parameters to detect early flap failure. Clin Hemorheol Microcirc 2011; 48: 81 - 94

[545] Lamby P, Prantl L, Schreml S et al. Improvements in high resolution ultrasound for postoperative investigation of capillary microperfusion after free tissue transfer. Clin Hemorheol Microcirc 2009; 43: 35 - 49

[546] Prantl L, Fellner C, Jung ME. Evaluation of Free Flap Perfusion with Dynamic Contrast-Enhanced Magnetic Resonance Imaging. Plastic and Reconstructive Surgery 2010; 126: 100e-1011e

[547] Lamby P, Prantl L, Fellner C et al. Post-operative monitoring of tissue transfers: advantages using contrast enhanced ultrasound(CEUS) and contrastMRI (ceMRI) with dynamic perfusion analysis? Clin Hemorheol Microcirc 2011; 48: $105-117$

[548] Selber JC, Garvey PB, Clemens MW et al. A Prospective Study of TransitTime Flow Volume Measurement for Intraoperative Evaluation and Optimization of Free Flaps. Plast Reconstr Surg 2013; 131: 270-281

[549] Mirzabeigi MN, Wang T, Kovach S] et al. Free Flap Take-Back following Postoperative Microvascular Compromise: Predicting Salvage versus Failure. Plast Reconstr Surg 2012; 130: 579- 589

[550] Reissig A, Copetti R, Mathis G et al. Lung Ultrasound in the Diagnosis and Follow-up of Community-Acquired Pneumonia: A Prospective, Multicenter, Diagnostic Accuracy Study. Chest 2012; 142: 965 - 972

[551] Nazerian P, Vanni S, Volpicelli G et al. Accuracy of Point-of-Care Multiorgan Ultrasonography for the Diagnosis of Pulmonary Embolism. Chest 2014; 145: $950-957$

[552] Volpicelli G, Silva F, Radeos M. Real-time lung ultrasound for the diagnosis of alveolar consolidation and interstitial syndrome in the emergency department. Eur J Emerg Med 2010; 17: 63-72

[553] Mathis G, Blank W, Reissig A et al. Thoracic Ultrasound for Diagnosing Pulmonary Embolism: A Prospective Multicenter Study of 352 Patients. Chest 2005; 128: $1531-1538$

[554] Sartori S, Postorivo S, Vece FD et al. Contrast-enhanced ultrasonography in peripheral lung consolidations: Whats its actual role? World J Radiol 2013; 5: $372-380$

[555] Bartelt S, Trenker C, Gorg C et al. Contrast-enhanced ultrasound of embolic consolidations in patients with pulmonary embolism: A pilot study. J Clin Ultrasound 2016; 44: 129-135

[556] Gorg C, Bert T, Kring R et al. Transcutaneous contrast enhanced sonography of the chest for evaluation of pleural based pulmonary lesions: experience in 137 patients. Ultraschall in Med 2006; 27: 437 - 444

[557] Linde HN, Holland A, Greene BH et al. Kontrastunterstzte Sonografie (CEUS) bei Pneumonie: Darstellungsmuster und prognostische Bedeutung eine retrospektive Studie bei 50 Patienten. Ultraschall in Med 2012; 33: $146-151$

[558] Sartori S, Nielsen I, Trevisani L et al. Contrast-Enhanced Sonography as Guidance for Transthoracic Biopsy of a Peripheral Lung Lesion With Large Necrotic Areas. J Ultrasound Med 2004; 23: 133-136

[559] Gorg C, Bert T, Kring R. Contrast-Enhanced Sonography of the Lung for Differential Diagnosis of Atelectasis. J Ultrasound Med 2006; 25: 35 39

[560] Tozer GM. Measuring tumour vascular response to antivascular and antiangiogenic drugs. Br J Radiol 2003; 76: S23-S35 
[561] Eisenhauer EA, Therasse P, Bogaerts ] et al. New response evaluation criteria in solid tumours: Revised RECIST guideline (version 1.1). Eur ] Cancer 2009; 45: 228-247

[562] Zhu AX, Holalkere NS, Muzikansky A et al. Early Antiangiogenic Activity of Bevacizumab Evaluated by Computed Tomography Perfusion Scan in Patients with Advanced Hepatocellular Carcinoma. Oncologist 2008; 13: $120-125$

[563] Dietrich CF, Averkiou MA, Correas JM et al. An introduction into dynamic contrast-enhanced ultrasound (DCE-US) for quantification of tissue perfusion. Ultraschall in Med 2012; 33: 344-351

[564] Peronneau P, Lassau N, Leguerney I et al. Contrast Ultrasonography: Necessity of Linear Data-áProcessing for the Quantification of Tumor Vascularization. Ultraschall in Med 2010; 31: 370-378

[565] Averkiou M, Lampaskis M, Kyriakopoulou K et al. Quantification of Tumor Microvascularity with Respiratory Gated Contrast Enhanced Ultrasound for Monitoring Therapy. Ultrasound Med Biol 2010; 36: $68-77$

[566] Williams R, Hudson JM, Lloyd BA et al. Dynamic Microbubble Contrastenhanced US to Measure Tumor Response to Targeted Therapy: A Proposed Clinical Protocol with Results from Renal Cell Carcinoma Patients Receiving Antiangiogenic Therapy. Radiology 2011; 260: 581 590

[567] Lassau N, Lamuraglia M, Vanel D et al. Doppler US with perfusion software and contrast medium injection in the early evaluation of isolated limb perfusion of limb sarcomas: prospective study of 49 cases. Ann Oncol 2005; 16: 1054-1060

[568] Cosgrove D, Lassau N. Imaging of perfusion using ultrasound. Eur ] Nucl Med Mol Imaging 2010; 37: 65-85

[569] Lamuraglia M, Escudier B, Chami L et al. To predict progression-free survival and overall survival in metastatic renal cancer treated with sorafenib: Pilot study using dynamic contrast-enhanced Doppler ultrasound. Eur J Cancer 2006; 42: 2472-2479
[570] Lassau N, Lamuraglia M, Chami L et al. Gastrointestinal Stromal Tumors Treated with Imatinib: Monitoring Response with Contrast-Enhanced Sonography. Am J Roentgenol 2006; 187: 1267-1273

[571] De Giorgi U, Aliberti C, Benea G et al. Effect of Angiosonography to Monitor Response During Imatinib Treatment in Patients with Metastatic Gastrointestinal Stromal Tumors. Clin Cancer Res 2005; 11: 6171-6176

[572] Escudier B, Lassau N, Angevin E et al. Phase I trial of sorafenib in combination with IFN alpsh-2a in patients with unresectable and/or metastatic renal cell carcinoma or malgnant melanoma. Clin Cancer Res 2007; 15: $1801-1809$

[573] Lassau N, Koscielny S, Albiges L et al. Metastatic Renal Cell Carcinoma Treated with Sunitinib: Early Evaluation of Treatment Response Using Dynamic Contrast-Enhanced Ultrasonography. Clin Cancer Res 2010; 16: $1216-1225$

[574] Lassau N, Chami L, Koscielny S et al. Quantitative functional imaging by Dynamic Contrast Enhanced Ultrasonography (DCE-US) in GIST patients treated with masatinib. Invest New Drugs 2012; 30: 765 - 771

[575] Lassau N, Koscienly S, Chami L et al. Advanced hepatocellular carcinoma: early evaluation of response to bevacizumab therapy at dynamic contrast-enhanced US with quantification - preliminary results. Radiology 2011; 258: $291-300$

[576] Lassau N, Chapotot L, Benatsou B et al. Standardization of Dynamic Contrast-Enhanced Ultrasound for the Evaluation of Antiangiogenic Therapies: The French Multicenter Support for Innovative and Expensive Techniques Study. Invest Radiol 2012; 47: 711 - 716

[577] Lassau N, Bonastre J, Kind M et al. Validation of Dynamic Contrast-Enhanced Ultrasound in Predicting Outcomes of Antiangiogenic Therapy for Solid Tumors: The French Multicenter Support for Innovative and Expensive Techniques Study. Invest Radiol 2014; 49: 794-800

[578] Casali PG, Blay JY, On behalf of the ESMO/CONTICANET/EUROBONET Consensus Panel of Experts. Gastrointestinal stromal tumours: ESMO Clinical Practice Guidelines for diagnosis, treatment and follow-up. Ann Oncol 2010; 21: v98-v102 